\title{
33. CORRELATION OF DARK AND LIGHT LAYERS, AND THE ORIGIN OF THEIR CYCLICITY IN THE QUATERNARY SEDIMENTS FROM THE JAPAN SEA ${ }^{1}$
}

\author{
Ryuji Tada, ${ }^{2}$ Itaru Koizumi, ${ }^{3}$ Adrian Cramp, ${ }^{4}$ and Atiur Rahman ${ }^{4}$
}

\begin{abstract}
Nearly continuous cores of Quaternary fine-grained sediments with distinct dark-light colored cycles were recovered from Sites 794, 795, and 797 in the basinal parts of the Japan Sea during Leg 127. A comparison of gray value (darkness) profiles supplemented by visual inspection of core photographs between sites indicated that most of the dark and light layers were correlatable between sites, and that two of the dark layers lie close to adjacent marker ash layers. These observations indicate that deposition of dark and light layers resulted from basin-wide synchronous events.

In order to understand the origin of these dark-light cycles, petrographical, mineralogical, compositional, and paleontological studies were carried out on closely spaced samples from the upper Quaternary sediments recovered from Site 797. Age model was constructed based on comparison between variation in diatom abundance and the standard oxygen isotope curve of Imbrie et al. (1984), the latter was interpolated between the five age controlled levels established at Site 797. The two curves show similar patterns which enabled us to "tune" the sediment ages to the oxygen isotope stages. We have to use variation in diatom abundance as a substitute for oxygen isotope curve since oxygen isotopic data are not available at the studied sites.

Bottom water oxygenation conditions were estimated based on two criteria: (1) the degree of lamina preservation and (2) the ratio of $\mathrm{C}_{\mathrm{org}}$ to $\mathrm{S}_{\text {tot }}$. The surface water productivity was deduced from the $\mathrm{C}_{\mathrm{org}}$ and biogenic silica content.

Results suggest that the bottom water oxygenation level and the surface water productivity varied significantly in response to the glacial-interglacial cycles. Glacio-eustatic sea-level changes and subsequent changes in water circulation in the Japan Sea appear to have been responsible for these variations and consequent changes in sediment composition throughout the Quaternary.
\end{abstract}

\section{INTRODUCTION}

The Japan Sea is a semienclosed marginal sea with an area of approximately $1,000,000 \mathrm{~km}^{2}$. It consists of two major depressions, the Japan and the Yamato Basins, which are separated by the Yamato Rise (Fig. 1). The water depth of the deep Japan Basin ranges from 3,000 to $3,700 \mathrm{~m}$, whereas that of the Yamato Basin ranges from 2,000 to 2,500 $\mathrm{m}$. The Japan Sea is connected to the Pacific Ocean through the Tsugaru Strait (130 m deep), to the Sea of Okhotsk through the Soya and Mamiya Straits ( 55 and $15 \mathrm{~m}$ deep, respectively), and to the East China Sea through the Tsushima Strait (130 m deep). The shallow sill depths of $130 \mathrm{~m}$, comparable in magnitude to the eustatic sea-level drop during the last glacial maximum(Fairbanks, 1989), suggest that glacio-eustatic sea-level fluctuations during the Quaternary could have had a significant effect on the oceanography within the Japan Sea.

In the present-day Japan Sea, potential temperature and salinity are more or less uniform at approximately $0^{\circ} \mathrm{C}$ and $34.1 \%$ o below the surface water layer ( $200-300 \mathrm{~m}$ thick), and the water is well oxygenated $\left(\mathrm{O}_{2}>210 \mu \mathrm{mol} / \mathrm{kg}\right)$ at all depths (Ohwada and Tanioka, 1971; Gamo et al., 1986). This is due to the formation of Japan Sea Proper Water (JSPW), which originates in the northern part of the Japan Sea during the winter (Hidaka, 1966). The water below the surface water layer can be subdivided into Deep Water and Bottom Water based on a sudden change in the temperature profile below 2,000-2,500 m depth (Gamo and Horibe, 1983). Bottom Water is characterized by a constant temperature (within $\pm 0.0005^{\circ} \mathrm{C}$ ) and dissolved oxygen levels (within $\pm 1 \mu \mathrm{mol} / \mathrm{kg}$ ). The thickness of the Bottom Water decreased by $400 \mathrm{~m}$ between 1977 and 1984, suggesting short term non-steady state abyssal circulation in the Japan Sea

Pisciotto, K. A., Ingle, J. C., Jr., von Breymann, M. T., Barron, J., et al., 1992. Proc, ODP, Sci. Results, 127/128, Pt. 1: College Station, TX (Ocean Drilling Program).

${ }^{2}$ Geological Institute, University of Tokyo, 7-3-1, Hongo, Tokyo 113, Japan.

${ }^{3}$ Department of Geology and Mineralogy, Hokkaido University, Kitaku, Sapporo 060, Japan.

${ }^{4}$ Department of Geology, University of Wales, College of Cardiff, P.O. Box 91, Cardiff CF1 3YE, United Kingdom.

Department of Geology and Geophysics, University of Utah, Salt Lake City, UT 8112-1183, U.S.A
(Gamo et al., 1986). The mean residence time of Bottom Water was calculated to be 300 years from analysis of ${ }^{14} \mathrm{C}$ data using a box model (Gamo and Horibe, 1983).

The warm Tsushima Current is the only current flowing into the Japan Sea. The current flows along the western margin of Honshu Island, the majority of the current then flows out through the Tsugaru Strait to the Pacific Ocean (Moriyasu, 1972). The surface water in the southern part of the Japan Sea is influenced by the Tsushima Current. Low-salinity, low-density water originating from the Eastern China Sea occurs in its upper part, and higher salinity, higher density water altered from the Kuroshio water mass occurs in its lower part (Moriyasu, 1972). In the northern part of the Japan Sea, the vertical variation in density is quite low (Fukuoka, 1962) which allows the formation of JSPW during the winter.

The present oceanographic conditions described here are probably regulated by the delicate balance of the amount and properties of waters flowing in and out of the Japan Sea through the narrow and shallow sills. Thus, it is expected that a glacio-eustatic sea-level change would have caused significant changes in the oceanographic conditions during the Quaternary.

Fine-grained siliciclastic sediments with distinct dark-light sedimentary cycles were recovered from the uppermost part of the sedimentary sequence at all four sites drilled during ODP Leg 127 in the Japan Sea. The sediments were classified as the lithologic Unit 1 based on the presence of the dark-light cycles (Tada and Iijima, this volume). Subsequent study of diatom stratigraphy by Koizumi (this volume) indicated that deposition of this unit started at approximately $2.6 \mathrm{Ma}$ at all four sites. Tada and Iijima (this volume) also noted that the dark-light cycles are distinct in the upper part of the unit (their Subunit 1A) deposition of which started at approximately $1.2 \mathrm{Ma}$, and are faint in the lower part of the unit (their Subunit 1B).

It has been known for some time that dark and light banded sediments are widely distributed in the Japan Sea (Ujiie and Ichikura, 1973; Ichikura and Ujiie, 1976; Oba, 1983; Kobayashi, 1985; Oba et al., 1991; Tanaka, in press). Oba et al. (1984, 1991) studied the paleontology and geochemistry of a 9.3-m-long piston core, which covers the last $85 \mathrm{ka}$, recovered from the Oki Ridge. They reported that a thick, laminated dark layer had been deposited during the last 


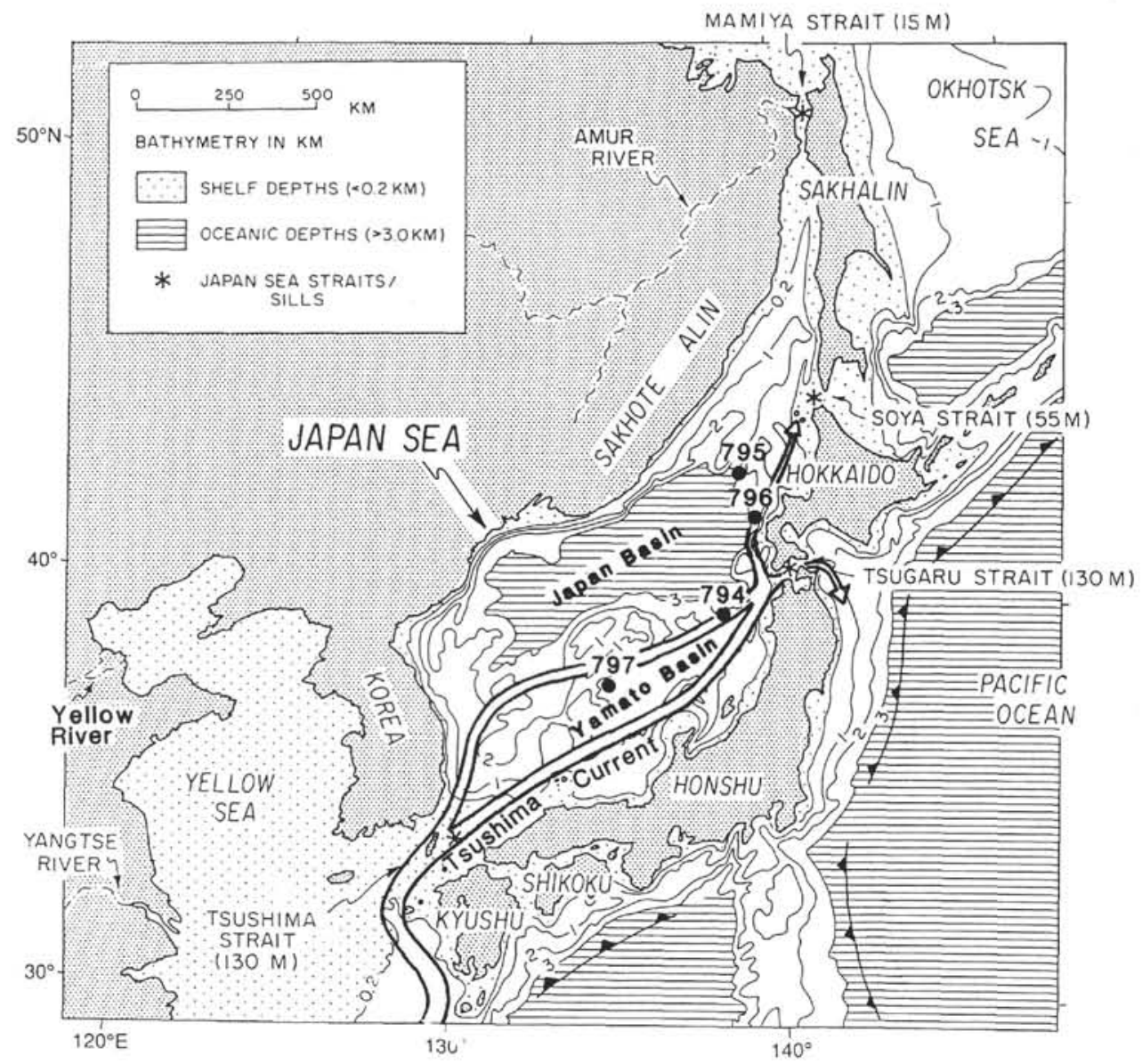

Figure 1. Map of the Japan Sea and its surrounding region showing the location of Sites 794 through 797 drilled during Leg 127. Bathymetry in kilometers. Isobath representing water depths $>3 \mathrm{~km}$ in the Pacific Ocean are not shown. Maximum water depths in the Japan Sea straits/sills are shown in parentheses.

glacial period and suggested that its deposition took place under a poorly oxygenated, possibly euxinic environment, these conditions were the result of a low-salinity surface water cap and the consequent reduction in vertical mixing.

Tanaka (in press) has reported the sedimentological aspect of the uppermost Quaternary fine-grained sediments from the Japan Sea. He found that the dark laminated layers are correlatable within the deeper parts of the Yamato and Tsushima Basins although their thickness varied considerably. He also noted the presence of normal graded sand and silt layers in the Toyama Deep Sea Fan area.

In this study, we aim to understand the origin of the rhythmical alternation of dark and light layers through sedimentological, mineralogical, and paleontological analyses of closely spaced samples (approximately every $35 \mathrm{~cm}$ ) from upper Quaternary sediments recovered at Site 797. In addition an attempt has been made to correlate the dark and light layers between Sites 794, 795, and 797. Our results suggest that the dark-light cycles within the upper Quaternary fine-grained sediments recovered from the Japan Sea are a record of basin wide paleoceanographic phenomena that are closely linked to glacio-eustatic sea-level changes.

\section{MATERIALS AND METHODS}

\section{Core Continuity and Depth Correction}

Core recovery exceeded $100 \%$ for most of the Quaternary sediments from Sites 794, 795, and 797, and drilling disturbance in these sediments is generally minimal. Apparent recovery exceeds $100 \%$ as a result of the expansion of sediments, but the amount of expansion estimated from core recovery is a minimum value since sediment may be missing between cores. We have no way to evaluate the thickness of the missing sediment between cores except for the interval between Cores 127-797B-1H and 127-797B-2H, which was recovered in Core $127-797 \mathrm{~A}-1 \mathrm{H}$. The thickness of the missing sediment is approximately $28 \mathrm{~cm}$. From the $5.9 \mathrm{~m}$ of core drilled and $5.85 \mathrm{~m}$ of recovery, the expansion ratio $1.04(=5.85 / 5.62)$ can be calculated for Core 127-797B-1H.

The core recovery from Quaternary and upper Pliocene sediments at Sites 794, 795, and 797 is generally between $102 \%$ and $105 \%$. If the expansion ratio of 1.04 is representative, then the thickness missing between cores is less than $20 \mathrm{~cm}$. Thus, although core splicing was not performed for any of the studied sites, the Quaternary and upper Pliocene sediments cored at Sites 794, 795, and 797 are probably nearly continuous.

We corrected the depth of Quaternary samples for expansion of the sediments where core recovery exceeded $100 \%$. We used the core recovery percent normalized by $100 \%$ as the expansion ratio. This correction tends to give the maximum depth for samples, which could exceed the true depth if sediments are missing between cores.

\section{Macroscopic Observation}

The on-board macroscopic observation of cores was supplemented later by the reexamination of core photographs and visual core description sheets recorded on board. The upper Quaternary fine-grained 
sediments from Sites 794, 795, and 797 were subdivided into dark, faintly dark, and light layers based on their relative darkness. In addition, sedimentary structures such as laminations, centimeterscale color banding, and bioturbation were described, and the thickness of each layer was measured for the sediments at Site 797. Macroscopic description of ash layers was also carried out, and the results are reported in Fujioka et al. (in preparation). Columnar sections were constructed based on macroscopic observation (Fig. 2).

\section{Gray Analysis}

Variation in the darkness of the sediments from Sites 794, 795, and 797 was quantified by determining the gray level on black-andwhite core photographs using a Shonic Graphic Analyzer. The graphic analyzer is equipped with an optical sensor tuned to emit a $700-\mathrm{nm}$ beam. The beam diameter of approximately $0.3 \mathrm{~cm}$ is equivalent to approximately $2 \mathrm{~cm}$ on the actual core surface. Measurement was carried out using a step width of $0.3 \mathrm{~cm}$ on the core photographs. The system was calibrated using black and white reference boards to obtain gray scale values (gray values) of 250 and 0 , respectively, before each core photo scan.

The gray value of the white board behind the core (background gray value) was measured on each black-and-white core photograph to check the difference in gray value among the core photographs caused by film or photographic paper exposure. The difference in background gray value is within \pm 4 for the core photographs from Site 795 and \pm 2 for those from Site 797. The difference is as large as \pm 11 for the core photographs from Site 794. We made no adjustment in gray values for the differences in the background gray values within the site except at Site 794. The average background gray values are 0 for core photographs from Site 797, and 8 for core photographs from Sites 794 and 795 . We did not make any adjustments for the difference between the sites.

There also is heterogeneity in background gray values within a core photograph. Generally, the center of a core photograph is lighter by $5-8$ gray values compared with its margin. We did not correct for these differences. Consequently, possible error in gray value within each site could be as large as \pm 8 .

\section{Sediment Composition for Samples from Hole 797B}

\section{Samples}

For the purposes of this study, 145 samples from Sections 127 797B-1H-1 to 127-797B-6H-2 were sampled at approximately $35 \mathrm{~cm}$ intervals and analyzed. First, a small portion of each sample was scooped with a toothpick to prepare a smear slide. Then, $10 \mathrm{~cm}^{3}$ of the sample was split in half. One half was analyzed for its assemblage of nannofossils and diatoms. The other half was washed twice with deionized water, centrifuged, and dried in an oven for 2-3 days at $60^{\circ} \mathrm{C}$. The dried portion was then powdered in an agate mortar and used for X-ray diffraction (XRD) and carbon and sulfur analyses.

\section{Smear Slide Analysis}

Smear slides were prepared for all samples. Special attention was taken to semiquantify the amount of diatoms which are the major constituents of biogenic silica. We chose a reference sample for which the diatom content was tentatively set as $30 \mathrm{vol} \%$ based on comparison with a visual chart. This reference sample contains $20 \pm 10 \mathrm{wt} \%$ opal-A based on quantification using XRD. The sample was then diluted with clay to prepare artificially mixed reference slides with diatom contents of 15 and $7.5 \mathrm{vol} \%$. The diatom content for each sample was determined under high magnification $(\times 400)$ by comparing with the reference slides. The contents of other components, such as silt grains, volcanic glass, radiolarians, silicoflagellates, sponge spicules, foraminifers, inorganic carbonate, organic matter, and pyrite, were determined by comparing with a visual chart. The content of clay was calculated by subtracting the content of these components from $100 \%$. The diameter of the largest quartz and plagioclase grains (the maximum grain size) was also measured for each sample.

The diatom number, defined as the number of diatoms per unit mass of the sediments (number/g), was counted based on the method described by Koizmi (1984). In this study, diatom number is used as a semiquantitative estimate of diatom abundance, which is independent of the diatom content. The diatom number is not necessarily equal to the diatom content since the size of diatom frustules is not uniform. Our results suggest that the diatom number is more sensitive to the change in the abundance of diatoms when the diatom content is less than a few percent.

\section{XRD Analysis}

Semiquantitative mineral analysis of the bulk samples was carried out using a Rigaku-Denki Geigerflex 4012. Powdered samples were mounted on a glass holder and X-rayed from $40^{\circ}$ to $2^{\circ} 2 \theta$ with $\mathrm{Cu}$ $\mathrm{K} \alpha$ radiation. Measurements were made at $30 \mathrm{kV}$ and $15 \mathrm{~mA}$ with a slit system of $1^{\circ}: 0.15^{\circ}: 1^{\circ}$, scanning speed of $4^{\circ} / \mathrm{min}$, and time constant of $1 \mathrm{~s}$. A control sample was run at least twice a day to check for any drift in the measurement conditions. Conditions remained quite stable during the entire course of measurements.

We used peak height as the quantitative measure of mineral content. High positive linear correlations with almost zero intercepts between carbonate carbon content vs. calcite peak height $\left(r^{2}=0.98\right)$ and total sulfur content vs. pyrite peak height $\left(r^{2}=0.83\right)$ suggests that the matrix effect is negligible for these samples, and that it is possible to quantify mineral content from peak height as far as crystallinity and chemical composition of the mineral are similar among the samples. Peak height reproducibility was better than $\pm 5 \%$.

For opal-A, for which the peak is extremely broad, peak height was determined by the height of its hump at $22^{\circ}$ relative to the background level, which is defined as the average of background intensities at $16^{\circ}$ and $39^{\circ}$. Tada and Iijima (this volume) derived the equation to quantify weight percent of opal-A from its peak height, and we slightly modified the equation as follows:

$$
\text { opal-A }(w t \%)=5.68 \times\{\text { opal-A }(\mathrm{cps} / 10)-1\} \text {. }
$$

The precision was within $\pm 10 \mathrm{wt} \%$. It should be noted that a broad hump at approximately $22^{\circ}$ is also caused by amorphous volcanic glass. Thus, estimation of opal-A by XRD could be misleading when the sediments contain significant amount of volcanic glass.

In this study, the detailed identification of clay mineral assemblages was not conducted because of time limitations. The peak at approximately $12.4^{\circ}$ was tentatively identified as kaolinite plus chlorite, the peak at $8.8^{\circ}$ as illite, and a rather broad peak at about $6^{\circ}$ as smectite. Because the second chlorite peak may overlap with the smectite peak and the crystallinity of smectite may change during diagenesis, its peak height is rather a poor representation of its amount.

\section{Carbon and Sulfur Analyses}

Total carbon and organic carbon $\left(\mathrm{C}_{\text {org }}\right)$ contents were analyzed using a Leco WR-12 carbon determinator. Approximately $0.1 \mathrm{~g}$ of powdered sample was oxidized at $1500^{\circ} \mathrm{C}$ for $55 \mathrm{~s}$ and the evolved $\mathrm{CO}_{2}$ gas was measured. For $\mathrm{C}_{\mathrm{org}}$ determination, $0.1 \mathrm{~g}$ of powdered sample was treated with $10 \% \mathrm{HCl}$ for one day, then dried at $60^{\circ} \mathrm{C}$ in a permeable crucible. Carbonate carbon $\left(\mathrm{C}_{\text {carb }}\right)$ content was calculated by the total carbon content minus the $\mathrm{C}_{\mathrm{org}}$. The precision was better than \pm 0.02 wt $\%$.

Total sulfur $\left(\mathrm{S}_{\text {tot }}\right)$ was analyzed using a Leco solid-state, automatic sulfur determinator. Approximately $0.1 \mathrm{~g}$ of powdered sample was oxidized at $1650^{\circ} \mathrm{C}$ for $15 \mathrm{~min}$ and the evolved $\mathrm{SO}_{2}$ gas was titrated. The precision was better than $\pm 0.05 \mathrm{wt} \%$ (Ogihara and Iijima, 1991). 


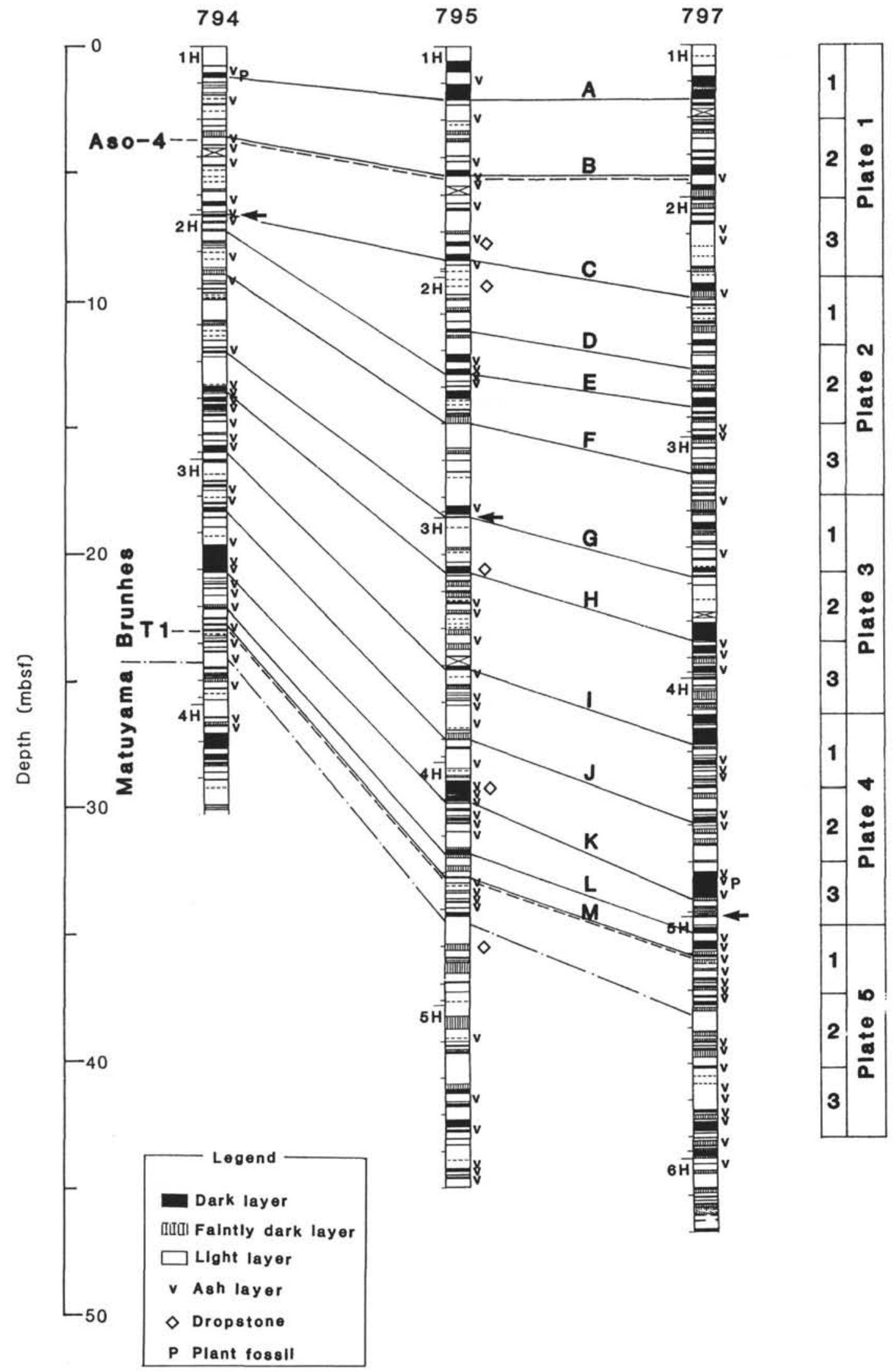

Figure 2. Columnar sections with correlation of the dark layers in the upper Quaternary sediments at Sites 794, 795, and 797. The thick and correlatable dark layers are labeled $\mathrm{A}$ through $\mathrm{M}$ in descending order. These layers are also shown in Figure 4 and Plates 1 through 5 . The core breaks where a stratigraphic gap is estimated are indicated by arrows. The stratigraphic intervals shown in Plates 1 through 5 are also indicated. 


\section{CHRONOLOGIC FRAMEWORK}

The sediments examined in this study are late Quaternary in age. Brown colored sediments, 50-60 cm thick, were recovered at the top of the first core at Sites 794, 795, and 797. This suggests that the top of the first core represent the surface sediments because the brown color is caused by oxidation near the sediment-water interface. The Brunhes/Matuyama magnetostratigraphic boundary was identified at 24.6, 35, and $38.4 \mathrm{~m}$ below seafloor (mbsf) at Sites 794, 795, and 797, respectively (Viggliotti and Wippern, pers. comm., 1990). These depths were corrected to $24.2,34.7$, and $38.3 \mathrm{mbsf}$. Within the sediments of Brunhes Chron, there are several additional biostratigraphic and tephrochronologic time controls.

Rahman (this volume) examined calcareous nannofossils and identified one nannofossil event within the Brunhes Chron that is useful to this work, the last occurrence (LO) of Pseudoemiliania lacunosa ( $0.46 \mathrm{Ma}$, after Thierstein et al., 1977). It occurred between 127-794A-1H-1, $74 \mathrm{~cm}$, and 127-794A-3H-2, $73 \mathrm{~cm}$, (between 0.74 and $18.42 \mathrm{mbsf}$ ) at Site 794 , between $127-795 \mathrm{~A}-3 \mathrm{H}-2,87 \mathrm{~cm}$, and 127-795A-4H-2, $46 \mathrm{~cm}$ (between 21.07 and $30.18 \mathrm{mbsf}$ ), at Site 795 , and between 127-797B-3H-6, $103 \mathrm{~cm}$, and 127-797B-3H-6, $135 \mathrm{~cm}$, (between 23.67 and $23.98 \mathrm{mbsf}$ ) at Site 797.

Koizumi (in preparation) examined diatoms and identified one diatom datum level, the LO of Rhizosolenia curvirostris $(0.30 \mathrm{Ma}$, after Koizumi, this volume) within the Brunhes Chron. He identified the level between $127-794 \mathrm{~A}-2 \mathrm{H}-1,80 \mathrm{~cm}$, and $127-794-2 \mathrm{H}-3,81 \mathrm{~cm}$ (between 7.56 and 10.43 mbsf), at Site 794, between 127-795A-2H-3, $29 \mathrm{~cm}$, and 127-795A-3H-1, $29 \mathrm{~cm}$ (between 12.48 and $19.08 \mathrm{mbsf}$ ), at Site 795, and between 127-797B-3H-1, $10 \mathrm{~cm}$, and 127-797B-3H$1,136 \mathrm{~cm}$ (between 16.41 and $16.72 \mathrm{mbsf}$ ), at Site 797.

Fujioka et al. (in preparation) examined the ash layers within the Quaternary sediments. They identified Aso-4 at all three sites, which is located at $127-794 \mathrm{~A}-1 \mathrm{H}-3,86 \mathrm{~cm}(3.86 \mathrm{mbsf})$, at Site 794, 127$795 \mathrm{~A}-1 \mathrm{H}-4,101 \mathrm{~cm}$ (5.51 mbsf), at Site 795, and 127-797B-1H-4, $98 \mathrm{~cm}$ (5.48 mbsf), at Site 797. Aso-4 ash was previously dated at $70 \mathrm{ka}$ (Machida, 1987), but recent oxygen isotopic studies of a core from the western North Pacific by Oba (1991) suggest that the age is between 86 and 90 ka because Aso-4 is situated within oxygen isotope stage 5.2. Fujioka et al. (in preparation) also showed that the ash layers located in 127-794A-3H-5, $91 \mathrm{~cm}$ (22.88 mbsf), and 127-797B-5H-2, $28 \mathrm{~cm}$ ( $36.12 \mathrm{mbsf}$ ), are correlatable based on the chemical composition of volcanic glass.

Although detailed tephrochronological studies have not yet been carried out, the ash layer at $127-795 \mathrm{~A}-\mathrm{H}, 35 \mathrm{~cm}$ (32.94 mbsf), is probably correlatable with this ash layer based on our visual inspection of core photographs. We tentatively named this ash layer $T_{1}$.

\section{RESULTS}

\section{Macroscopic Observation}

The thickness of dark, faintly dark, and light layers in the upper Quaternary fine-grained sediments from Sites 794, 795, and 797 varied ranging from a few centimeters to more than a meter. The darkness rank of the layers from which analyzed samples were taken at Site 797 is listed in Table 1 together with the thickness of the layers.

Some of dark and faintly dark layers have a yellow hue, contain foraminifers, and have a rough texture on their wire-cut surfaces, whereas other dark and faintly dark layers have a neutral to greenish hue and have a smooth appearance. Light layers tend to have a neutral hue but may occasionally have a yellow hue and their wire-cut surfaces are either smooth or rough in texture. The bottom contacts of the dark and faintly dark layers are generally sharp, whereas their upper contacts are either sharp or gradational.

Parallel lamination, color banding, and bioturbation are the dominant sedimentary structures recognized in the sediments studied. Individual parallel lamina is less than a few millimeters thick and can be recognized by the slight difference in color or texture on wire-cut surfaces. When dark layers contain foraminifer tests, lamination is easily recognizable. Color bands are $0.5-1 \mathrm{~cm}$ thick and show parallel banding. In dark layers and faintly dark layers, they tend to be recognized as a contrast in darkness, whereas they are recognized as a contrast in hue, darkness, and texture in light layers. Greenish color bands with rough wire-cut surface are common in light layers.

Bioturbation is recognized by either a mottled appearance or vague and small ( $<5 \mathrm{~mm}$ diameter) burrows vertical or oblique to the bedding. In addition to the bioturbated sediments, there are homogeneous sediments without any observable sedimentary structures. It is not certain whether sedimentary structures were erased by intense bioturbation or they were originally absent. However, the wire-cut surfaces of the homogeneous sediments are very smooth, which is different from heavily bioturbated sediments whose wire-cut surfaces tend to be rough in texture.

On the basis of sedimentary structure, samples from Site 797 were classified as being laminated, color-banded, homogeneous, or bioturbated. Results are listed in Table 1. Figure 3 shows the relationship between the darkness rank and the sedimentary structures for samples from Site 797. Dark and faintly dark layers are generally well laminated or color banded though rarely homogeneous, whereas light layers are either homogeneous, color banded, or bioturbated though rarely laminated.

Very well-rounded pebbles of volcanic rocks (mostly andesitic), as large as $5 \mathrm{~cm}$ in the long diameter were found within the sediments at Site 795. We interpreted them as dropstones. The dropstones tend to occur within dark layers or at the boundary between dark and light layers in the upper Quaternary sediments.

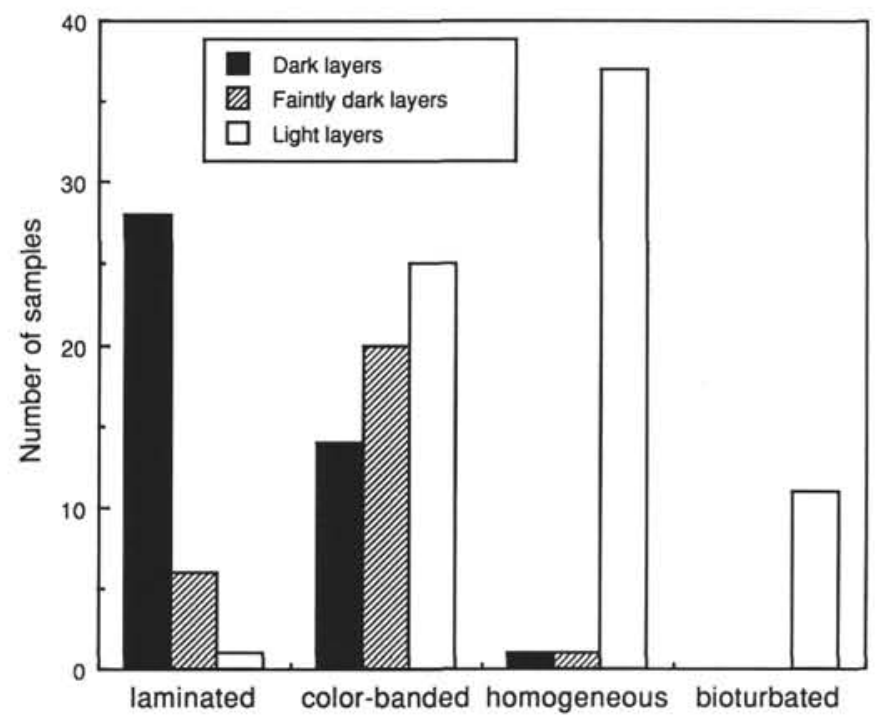

Figure 3 . The relationship between sedimentary structure and the darkness of layers.

Carbonaceous plant fragments, a few centimeters in length, were identified in the dark layers at Sites 794 and 797.

The vertical distribution of the dark, faintly dark, and light layers, together with ash layers, dropstones, and plant fragments, is summarized in the columnar sections (Fig. 2).

\section{Gray Analysis}

The vertical profiles of the gray values for Sites 794, 795, and 797 are shown in Figure 4 . The variation in gray value is smoothed by taking a 5-point moving average. The gray value, which increases with increasing darkness, ranges from 50 to 230 at Site 794,110 to 
Table 1. Macroscopic and smear slide analysis of the upper Quaternary samples from Hole 797B.

\begin{tabular}{|c|c|c|c|c|c|c|c|c|c|c|c|c|c|c|c|c|c|c|}
\hline $\begin{array}{l}\text { Core, section, } \\
\text { interval (cm) }\end{array}$ & $\begin{array}{l}\text { Depth } \\
\text { (mbsn) }\end{array}$ & $\begin{array}{l}\text { Sedimentary } \\
\text { structure }\end{array}$ & $\begin{array}{c}\text { Darkness } \\
\text { tank }\end{array}$ & $\begin{array}{c}\text { Layer } \\
\text { thickness } \\
\text { (cm) }\end{array}$ & $\begin{array}{c}\text { Silt } \\
\text { (vol\%) }\end{array}$ & $\begin{array}{l}\text { Clay } \\
\text { (volf) }\end{array}$ & $\begin{array}{l}\text { GGass } \\
\text { (voly) }\end{array}$ & $\begin{array}{l}\text { Diatom } \\
\text { (voly) }\end{array}$ & $\begin{array}{c}\text { Radiolaria } \\
\text { (voly) }\end{array}$ & $\begin{array}{c}\text { Silicoflagellate } \\
\text { (volis) }\end{array}$ & $\begin{array}{c}\text { Foraminififer } \\
\text { (vol\%) }\end{array}$ & $\begin{array}{l}\text { Inorganic } \\
\text { carbonate } \\
\text { (vols) }\end{array}$ & $\begin{array}{l}\text { Organic } \\
\text { matter } \\
\text { (vol\%) }\end{array}$ & $\begin{array}{l}\text { Pyrite } \\
\text { (vol\%) }\end{array}$ & $\begin{array}{l}\text { Sponge } \\
\text { (volw) }\end{array}$ & $\begin{array}{l}\text { Total } \\
\text { matter } \\
\text { (vols) }\end{array}$ & $\begin{array}{l}\text { Diatom } \\
\text { number } \\
\left(10^{7} / g\right)\end{array}$ & $\begin{array}{c}\text { Maximum } \\
\text { grain size }(\mu \mathrm{m})\end{array}$ \\
\hline \multicolumn{19}{|l|}{ 127-7978- } \\
\hline $1 \mathrm{H}-1,13-15$ & 0.13 & homogeneous & (brown) & 50 & 25 & 50 & 0 & 15 & 1 & 3 & 0 & 0 & 1 & 2 & 3 & 100 & 2.570 & 108 \\
\hline & 0.43 & homogeneous & (brown) & so & 30 & 55 & 0 & 6 & i & 0 & 0 & 0 & 3 & 3 & 2 & 100 & 0.770 & 83 \\
\hline $1 \mathrm{H}-1.74-76$ & 0.71 & homogeneous & light & 35 & 25 & 60 & i & 6 & i & 0 & 1 & 0 & 3 & i & 2 & 100 & 1.190 & 91 \\
\hline IH-1. $134-136$ & 1.29 & color-banded & dark & 45 & 40 & 20 & 2 & 2 & 0 & 7 & i & 3 & 5 & II & 0 & 100 & 1.290 & 166 \\
\hline IH-2, 13-15 & 1.56 & color-banded & dark & 45 & 40 & & 2 & 5 & 1 & 0 & 2 & 10 & 6 & 11 & 2 & 100 & 0.120 & 158 \\
\hline IH-2, 44-46 & 1.86 & color-banded & dark & 20 & 35 & 40 & 1 & 0 & 0 & 0 & 1 & 10 & 3 & 10 & 0 & 100 & 0.060 & 66 \\
\hline $\begin{array}{l}1 \mathrm{H}-2,74-76 \\
\mathrm{H}-2\end{array}$ & $\begin{array}{l}2.14 \\
2.43\end{array}$ & $\begin{array}{l}\text { color-banded } \\
\text { elor-anded }\end{array}$ & light & & $\begin{array}{l}50 \\
30\end{array}$ & $\begin{array}{l}30 \\
60\end{array}$ & $2_{2}^{2}$ & 0 & 0 & 0 & 0 & 7 & 3 & 8 & 0 & 100 & 0.020 & 199 \\
\hline $\begin{array}{l}\text { IH-2.104-1060 } \\
\text { IH-3,13-15 }\end{array}$ & $\begin{array}{l}2.43 \\
2.99\end{array}$ & $\begin{array}{l}\text { color-banded } \\
\text { homogeneous }\end{array}$ & $\begin{array}{l}\text { light } \\
\text { light }\end{array}$ & 22 & $\begin{array}{l}30 \\
30\end{array}$ & $\begin{array}{l}60 \\
50\end{array}$ & ${ }_{2}^{2}$ & $\begin{array}{l}0 \\
3\end{array}$ & $\begin{array}{l}0 \\
0\end{array}$ & $\begin{array}{l}0 \\
0\end{array}$ & $\begin{array}{l}0 \\
0\end{array}$ & $\begin{array}{l}1 \\
3\end{array}$ & ${ }_{3}^{3}$ & 4 & $\begin{array}{l}0 \\
2\end{array}$ & $\begin{array}{l}100 \\
100\end{array}$ & $\begin{array}{l}0.030 \\
0070\end{array}$ & 125 \\
\hline $\mathrm{IH}-3,4-46$ & $\begin{array}{l}2.99 \\
3.29\end{array}$ & $\begin{array}{l}\text { nomogeneneous } \\
\text { color-banded }\end{array}$ & $\begin{array}{l}\text { lignt } \\
\text { dark }\end{array}$ & $\begin{array}{l}10 \\
2\end{array}$ & $\begin{array}{l}30 \\
40\end{array}$ & $\begin{array}{l}30 \\
45\end{array}$ & $\frac{2}{2}$ & 1 & $\begin{array}{l}0 \\
0\end{array}$ & 0 & 0 & 2 & 4 & 4 & $\frac{2}{2}$ & 100 & 0.020 & 208 \\
\hline $1 \mathrm{H}-3,74-76$ & 3.57 & color-banded & light & 19 & 55 & 30 & 2 & 0 & 0 & 0 & 0 & 1 & 5 & 7 & 0 & 100 & 0.010 & 241 \\
\hline $1 \mathrm{H}-3,104-106$ & 3.86 & homogeneous & light & 32 & 40 & 45 & i & 0 & 0 & 0 & 0 & 4 & 3 & 7 & 0 & 100 & 0.020 & 208 \\
\hline $1 \mathrm{H}-3.134-136$ & 4.14 & color-banded & dark & 7 & 35 & 40 & 0 & 0 & 0 & 0 & 0 & 5 & 5 & 15 & 0 & 100 & 0.430 & 83 \\
\hline $1 \mathrm{H}-4,13-15$ & 4.42 & laminated & dark & 9 & 30 & so & 1 & 2 & 0 & 0 & 0 & 2 & 3 & 10 & 2 & 100 & 1.700 & 100 \\
\hline IH- $4,44-46$ & 4.71 & homogeneous & dark & 43 & 35 & 45 & 0 & 10 & 1 & 0 & 0 & 1 & 2 & 3 & 3 & 100 & 1.100 & 91 \\
\hline 1H-4.71-73 & 4.97 & laminated & dark & 43 & 25 & 45 & 1 & 16 & 0 & 0 & 0 & 1 & 2 & 8 & 2 & 100 & 1.470 & 66 \\
\hline IH-4, 104-106 & 5.29 & color-banded & light & 35 & 35 & 40 & 2 & 0 & 0 & 0 & 3 & 15 & 2 & 3 & 0 & 100 & 0.160 & 100 \\
\hline $2 \mathrm{H}-1,14-16$ & $\begin{array}{l}6.05 \\
6.34\end{array}$ & color-banded & faintly dark & 10 & 35 & 45 & 2 & 5 & 0 & 0 & 0 & 0 & 3 & 8 & 2 & 100 & 0.640 & 116 \\
\hline $\begin{array}{l}2 \mathrm{H}-\mathrm{l}, 44,46 \\
2 \mathrm{H}-1,102-104\end{array}$ & $\begin{array}{l}6.34 \\
690\end{array}$ & $\begin{array}{c}\text { color-banded } \\
\text { laminated }\end{array}$ & $\begin{array}{l}\text { faintly yark } \\
\text { dark }\end{array}$ & $\begin{array}{l}20 \\
12\end{array}$ & $\begin{array}{r}25 \\
10\end{array}$ & $\begin{array}{l}42 \\
25\end{array}$ & ${ }_{1}^{3}$ & 15 & i & $\begin{array}{l}0 \\
0\end{array}$ & $0^{\circ}$ & 10 & ${ }_{5}^{4}$ & 6 & ${ }^{3}$ & 100 & $\begin{array}{l}0.770 \\
\end{array}$ & 91 \\
\hline $2 \mathrm{H}-1,134-136$ & $\begin{array}{l}6.90 \\
7.21\end{array}$ & $\begin{array}{l}\text { laminatited } \\
\text { color-banded }\end{array}$ & $\begin{array}{l}\text { dark } \\
\text { light }\end{array}$ & 75 & $\begin{array}{l}10 \\
33\end{array}$ & 35 & $\frac{1}{2}$ & $\begin{array}{l}20 \\
15\end{array}$ & 2 & 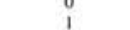 & 20 & 1 & i & 7 & ${ }_{3}^{0}$ & 100 & $\begin{array}{l}1.340 \\
1.030\end{array}$ & $\begin{array}{l}108 \\
75\end{array}$ \\
\hline $2 \mathrm{H}-2,14-16$ & 7.50 & homogeneous & $\begin{array}{l}\text { light } \\
\text { light }\end{array}$ & 75 & 25 & 25 & 1 & 30 & 3 & 2 & 0 & 3 & 2 & 6 & 3 & 100 & 1.710 & 116 \\
\hline $2 \mathrm{H}-2,44-46$ & 7.79 & $\begin{array}{l}\text { nothogeneneous } \\
\text { homogeneous }\end{array}$ & $\begin{array}{l}\text { lugnt } \\
\text { light }\end{array}$ & 42 & 25 & 25 & 2 & $\begin{array}{l}300 \\
30\end{array}$ & 1 & 2 & 0 & 2 & 2 & $\begin{array}{l}0 \\
8\end{array}$ & 3 & 100 & 1.290 & 83 \\
\hline $2 \mathrm{H}-2,74-76$ & 8.08 & homogeneous & light & 42 & 30 & 50 & 5 & 7 & $i$ & 0 & 0 & 0 & 3 & 3 & i & 100 & 0.510 & 116 \\
\hline $2 \mathrm{H}-2,102-104$ & 8.35 & bioturbated & light & 38 & 35 & 40 & 5 & 3 & 0 & 0 & 0 & 7 & 4 & 6 & 0 & 100 & 0.030 & 116 \\
\hline $2 \mathrm{H}-2,134-136$ & 8.66 & laminated & dark & 8 & 40 & 35 & 5 & 2 & 0 & 0 & 0 & 3 & 3 & 10 & 2 & 100 & 0.030 & 149 \\
\hline $2 \mathrm{H}-3,14-16$ & 8.95 & color-banded & light & 13 & 40 & 40 & 5 & 1 & 0 & 0 & 0 & 3 & 3 & 8 & 0 & 100 & 0.010 & 166 \\
\hline $2 \mathrm{H}-3,44,46$ & 9.24 & color-banded & light & 30 & 45 & 40 & 3 & 0 & 0 & 0 & 0 & 2 & 2 & 8 & 0 & 100 & 0.020 & 100 \\
\hline $2 \mathrm{H}-3,74-76$ & $\begin{array}{l}9.53 \\
0.80\end{array}$ & laminated & dark & 32 & 30 & 35 & 3 & 0 & & 0 & 2 & 5 & 5 & 20 & 0 & 100 & 0.020 & 216 \\
\hline $\begin{array}{l}2 \mathrm{H}-3,102-104 \\
2 \mathrm{H}-134\end{array}$ & 9.80 & color-banded & faintly dark & 25 & 50 & 25 & 1 & 3 & 0 & 0 & 0 & 3 & 2 & 15 & 1 & 100 & 0.060 & 149 \\
\hline $2 \mathrm{H}-3.134-136$ & 10.11 & color-banded & light & 199 & ${ }_{36}^{36}$ & 45 & 3 & 1 & 0 & 0 & 0 & 2 & 4 & 7 & 2 & 100 & 0.080 & 158 \\
\hline $2 \mathrm{H}-4,14-16$ & 10.40 & color-banded & light & 15 & 35 & 45 & 1 & 0 & 0 & 0 & 0 & 3 & 3 & 12 & 1 & 100 & 0.010 & 166 \\
\hline $2 \mathrm{H}-4,44-46$ & 10.69 & color-banded & faintly dark & 7 & 35 & 45 & 2 & 3 & 0 & 0 & 0 & 2 & 3 & 8 & 2 & 100 & 0.040 & 66 \\
\hline $\begin{array}{l}2 \mathrm{H}-4,74-76 \\
2 \mathrm{H}-4\end{array}$ & $\begin{array}{l}10.98 \\
11.26\end{array}$ & laminated & $\begin{array}{l}\text { faintly dark } \\
\text { fainty }\end{array}$ & $\begin{array}{l}35 \\
35\end{array}$ & $\begin{array}{l}35 \\
35\end{array}$ & 47 & $\frac{1}{2}$ & 0 & 0 & 0 & 0 & 3 & 2 & 12 & 0 & 100 & 0.003 & 199 \\
\hline $2 \mathrm{H}-4,102-104$ & 11.26 & color-banded & faintly dark & 35 & 35 & 55 & 2 & 0 & 0 & 0 & 0 & 2 & 1 & 4 & 1 & 100 & 0.003 & 100 \\
\hline $\begin{array}{l}2 \mathrm{H}-4,134-136 \\
2 \mathrm{H}-4,44-46\end{array}$ & $\begin{array}{l}11.57 \\
12.15\end{array}$ & color-banded & dark & 25 & 40 & 40 & 2 & ${ }^{3}$ & 0 & 0 & 0 & $\frac{1}{5}$ & 3 & 8 & 3 & 100 & 0.450 & 141 \\
\hline $\begin{array}{l}2 \mathrm{H}-5,44.46 \\
2 \mathrm{H}-5,74-76\end{array}$ & $\begin{array}{l}12.15 \\
12.44\end{array}$ & $\begin{array}{l}\text { homogeceneous } \\
\text { color-banded }\end{array}$ & $\begin{array}{l}\text { light } \\
\text { faintly dark }\end{array}$ & $\begin{array}{l}100 \\
35\end{array}$ & ${ }_{28}^{25}$ & $\begin{array}{l}50 \\
35\end{array}$ & $\frac{2}{2}$ & $\begin{array}{l}8 \\
25\end{array}$ & $\begin{array}{l}0 \\
0 \\
0\end{array}$ & $\begin{array}{l}0 \\
0\end{array}$ & $\begin{array}{l}0 \\
0\end{array}$ & $\begin{array}{l}5 \\
0\end{array}$ & $\begin{array}{l}2 \\
5\end{array}$ & $\begin{array}{l}5 \\
3\end{array}$ & ${ }_{2}^{3}$ & $\begin{array}{l}100 \\
100\end{array}$ & 0.200 & 100 \\
\hline $\begin{array}{l}2 \mathrm{H}-3,74-16 \\
2 \mathrm{H}-5,102-104\end{array}$ & $\begin{array}{l}12.44 \\
12.71\end{array}$ & $\begin{array}{l}\text { color-banded } \\
\text { homogeneous }\end{array}$ & $\begin{array}{l}\text { laintly } \\
\text { light }\end{array}$ & 10 & ${ }_{30}^{28}$ & 35 & 3 & 15 & 2 & $\begin{array}{l}0 \\
0\end{array}$ & $\begin{array}{l}0 \\
0\end{array}$ & $\begin{array}{l}0 \\
4\end{array}$ & 3 & 5 & 3 & 100 & 1.700 & 125 \\
\hline $2 \mathrm{H}-5,134-136$ & 13.02 & homogeneous & $\begin{array}{l}\text { lignt } \\
\text { light }\end{array}$ & 18 & 30 & 38 & i & 17 & $i$ & 0 & 0 & i & 5 & 3 & 4 & 100 & 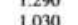 & $\begin{array}{l}133 \\
83\end{array}$ \\
\hline $2 \mathrm{H}-6,14-16$ & 13.31 & color-banded & light & is & 45 & 40 & 1 & 5 & 0 & 0 & 0 & 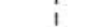 & 3 & 2 & 3 & 100 & 0.040 & $\begin{array}{l}83 \\
58\end{array}$ \\
\hline $2 \mathrm{H}-6,44-46$ & 13.60 & laminated & dark & 14 & 30 & 38 & $i$ & 15 & 0 & 0 & 0 & 2 & 5 & 8 & $i$ & & 1.290 & 58 \\
\hline $2 \mathrm{H}-6,74-76$ & 13.89 & laminated & dark & 36 & 35 & 40 & i & 2 & 0 & 0 & 0 & 2 & 4 & 15 & $\mathrm{i}$ & 100 & 0.040 & 100 \\
\hline $2 \mathrm{H}-6,134-136$ & 14.47 & color-banded & light & 11 & 35 & 40 & 1 & 8 & 0 & 0 & 1 & 3 & 2 & 9 & 1 & 100 & 0.390 & 208 \\
\hline $2 \mathrm{H}-7,14-16$ & 14.76 & laminated & faintly dark & 12 & 35 & 40 & 1 & 14 & 1 & 0 & 0 & 1 & 1 & 4 & 3 & 100 & 0.510 & 75 \\
\hline $2 \mathrm{H}-7,44-46$ & 15.05 & laminated & faintly dark & 32 & 30 & 35 & 2 & 20 & 1 & 0 & 0 & 1 & 2 & 7 & 2 & 100 & 0.770 & 91 \\
\hline $3 \mathrm{H}-1,14-16$ & 15.55 & color-banded & faintly dark & 16 & 40 & 45 & 2 & 1 & 0 & 0 & 0 & 2 & 3 & 6 & 1 & 100 & 0.030 & 208 \\
\hline $3 \mathrm{H}-1,44-46$ & 15.84 & color-banded & faintly dark & 5 & 37 & so & $i$ & 0 & 0 & 0 & 0 & 3 & 3 & 5 & 1 & 100 & 0.020 & 415 \\
\hline $3 \mathrm{H}-1,74-76$ & 16.13 & homogeneous & light & 27 & 38 & so & 3 & 0 & 0 & 0 & 0 & 1 & 1 & 7 & 0 & 100 & 0.010 & 249 \\
\hline $3 \mathrm{H}-1,103-105$ & 16.41 & color-banded & faintly dark & 10 & 35 & 45 & 1 & 8 & 0 & 0 & 0 & 2 & 2 & 5 & 2 & 100 & 0.150 & 50 \\
\hline $3 \mathrm{H}-1,135-137$ & $\begin{array}{l}16.72 \\
1700\end{array}$ & color-banded & dark & 18 & 20 & 68 & 0 & 0 & 0 & 0 & 0 & 1 & 3 & 7 & 1 & 100 & 0.350 & 100 \\
\hline $3 \mathrm{H}-2,14-16$ & $\begin{array}{l}17.00 \\
0\end{array}$ & bioturbated & light & 28 & 35 & 50 & 1 & 0 & 0 & 0 & 0 & 2 & 2 & 10 & 0 & 100 & 0.080 & 208 \\
\hline $\begin{array}{l}3 \mathrm{H}-2,444,46 \\
3 \mathrm{H}, 2,7-76\end{array}$ & $\begin{array}{l}17.29 \\
17.58\end{array}$ & homogeneous & light & 12 & 37 & 47 & 1 & 0 & 0 & 0 & 0 & 2 & 2 & 10 & 1 & 100 & 0.050 & 249 \\
\hline $3 \mathrm{H}-2,74-76$ & 17.38 & color-banded & lainly dark & & 30 & 56 & & & & & 0 & 1 & & $?$ & 2 & 100 & 0.130 & 133 \\
\hline $3 \mathrm{H}-2,103-105$ & 17.86 & homogeneous & light & 15 & 32 & so & 1 & 5 & 0 & 0 & 0 & t & 1 & 7 & 3 & 100 & 0.590 & 66 \\
\hline $3 \mathrm{H}-2,135-137$ & 18.17 & color-banded & faintly dark & 33 & 27 & 40 & 1 & 15 & 1 & 1 & 0 & 1 & 3 & 10 & 1 & 100 & 1.030 & 75 \\
\hline $3 \mathrm{H}-3.14-16$ & $\begin{array}{l}18.45 \\
1871\end{array}$ & color-banded & $\begin{array}{l}\text { faintly dark } \\
\text { flobth }\end{array}$ & $\begin{array}{l}10 \\
30\end{array}$ & 33 & 40 & $\frac{1}{2}$ & 3 & 0 & 0 & 3 & 5 & 4 & 10 & 1 & 100 & 0.240 & 125 \\
\hline $\begin{array}{l}3 \mathrm{H}-3,40-42 \\
3 \mathrm{H}-3,74-76\end{array}$ & $\begin{array}{l}18.71 \\
1904\end{array}$ & $\begin{array}{l}\text { bioturbated } \\
\text { laminated }\end{array}$ & $\begin{array}{l}\text { light } \\
\text { dark }\end{array}$ & $\begin{array}{l}30 \\
33\end{array}$ & $\begin{array}{l}30 \\
40\end{array}$ & $\begin{array}{l}50 \\
35\end{array}$ & ${ }_{2}^{2}$ & 9 & 1 & 1 & ${ }_{3}^{0}$ & ${ }_{4}^{0}$ & $\frac{1}{4}$ & ${ }_{10}^{4}$ & $2_{1}^{2}$ & 100 & $\begin{array}{l}0.570 \\
0.030\end{array}$ & $\begin{array}{l}108 \\
249\end{array}$ \\
\hline $\begin{array}{l}\begin{array}{l}3 \mathrm{H}-3,7,74-76 \\
3 \mathrm{H}-3,103-105\end{array}\end{array}$ & $\begin{array}{l}19.04 \\
19.32\end{array}$ & $\begin{array}{l}\text { laminated } \\
\text { laminated }\end{array}$ & $\begin{array}{l}\text { dark } \\
\text { faintly dark }\end{array}$ & $\begin{array}{l}33 \\
15\end{array}$ & $\begin{array}{l}40 \\
37\end{array}$ & $\begin{array}{l}35 \\
50\end{array}$ & ${ }_{0}^{2}$ & $\begin{array}{l}1 \\
0\end{array}$ & $\begin{array}{l}0 \\
0\end{array}$ & $\begin{array}{l}0 \\
0\end{array}$ & ${ }_{0}^{3}$ & $\begin{array}{l}4 \\
3\end{array}$ & $\begin{array}{l}4 \\
3\end{array}$ & $\begin{array}{l}100 \\
7\end{array}$ & $\begin{array}{l}1 \\
0\end{array}$ & $\begin{array}{l}100 \\
100\end{array}$ & $\begin{array}{l}0.030 \\
0.010\end{array}$ & 249 \\
\hline $3 \mathrm{H}-3,135-137$ & $\begin{array}{l}19.32 \\
19.63\end{array}$ & $\begin{array}{l}\text { lamininated } \\
\text { color-banded }\end{array}$ & $\begin{array}{l}\text { fainlly dark } \\
\text { light }\end{array}$ & ${ }_{3}^{15}$ & $\begin{array}{l}37 \\
40\end{array}$ & $\begin{array}{l}50 \\
36\end{array}$ & $\begin{array}{l}0 \\
0\end{array}$ & $\begin{array}{l}0 \\
0\end{array}$ & $\begin{array}{l}0 \\
0\end{array}$ & $\begin{array}{l}0 \\
0\end{array}$ & $\begin{array}{l}0 \\
0\end{array}$ & $\frac{3}{2}$ & $\begin{array}{l}3 \\
2\end{array}$ & 20 & $\begin{array}{l}0 \\
0\end{array}$ & $\begin{array}{l}100 \\
100\end{array}$ & $\begin{array}{l}0.010 \\
0.003\end{array}$ & $\begin{array}{l}1235 \\
415\end{array}$ \\
\hline $3 \mathrm{H}-4,14-16$ & 19.91 & homogeneneous & $\begin{array}{l}\text { lignt } \\
\text { light }\end{array}$ & 35 & 35 & $\begin{array}{l}30 \\
53\end{array}$ & 2 & 0 & 0 & 0 & 0 & 2 & 2 & ${ }_{6}^{20}$ & 0 & 100 & 0.030 & 141 \\
\hline $3 \mathrm{H}-4,44,46$ & 20.20 & color-banded & dark & 4 & 30 & 53 & $i$ & 2 & 0 & 0 & 0 & $i$ & 4 & 7 & 2 & 100 & 0.040 & 66 \\
\hline $3 \mathrm{H}-4,74-76$ & 20.49 & homogeneous & light & 20 & 25 & 60 & 2 & 0 & 0 & 0 & 0 & 4 & 1 & 7 & i & 100 & 0.090 & 66 \\
\hline $3 \mathrm{H}-4,103-105$ & 20.77 & color-banded & light & 12 & 28 & 45 & 6 & 8 & 0 & 0 & 1 & 2 & 2 & 6 & 2 & 100 & 1.030 & 133 \\
\hline $3 \mathrm{H}-4,135-137$ & 21.08 & color-banded & light & 23 & 33 & 45 & 2 & 10 & 0 & 0 & 0 & 0 & 1 & 7 & 2 & 100 & 0.210 & 266 \\
\hline $3 \mathrm{H}-5,14-16$ & 21.36 & bioturbated & light & 55 & 28 & 55 & 4 & 3 & 0 & 0 & 0 & 0 & 1 & 7 & 2 & 100 & 0.480 & 100 \\
\hline $\begin{array}{l}3 \mathrm{H} \cdot 5,44-46 \\
34-5,72-75\end{array}$ & 21.65 & homogeneous & light & 55 & 25 & 60 & 2 & 5 & 1 & 0 & 0 & 0 & 1 & 4 & 2 & 100 & 0.210 & 149 \\
\hline $3 \mathrm{H} \cdot 5,73-75$ & 21.93 & bioturbated & light & 92 & 28 & 55 & 2 & 10 & 0 & 0 & 0 & $\begin{array}{lll}0 & & \end{array}$ & 1 & 3 & 1 & 100 & 0.210 & 50 \\
\hline
\end{tabular}


Table 1 (continued).

\begin{tabular}{|c|c|c|c|c|c|c|c|c|c|c|c|c|c|c|c|c|c|c|}
\hline $\begin{array}{l}\text { Core, section. } \\
\text { interval (cm) }\end{array}$ & $\begin{array}{l}\text { Depth } \\
\text { (mbst) }\end{array}$ & $\begin{array}{l}\text { Sedimentary } \\
\text { structure }\end{array}$ & $\begin{array}{c}\text { Darkness } \\
\text { rank }\end{array}$ & $\begin{array}{c}\text { Layer } \\
\text { thickness } \\
\text { (cm) }\end{array}$ & $\begin{array}{c}\text { Silt } \\
\text { (vol\%) }\end{array}$ & $\begin{array}{c}\text { Clay } \\
\text { (vol\%) }\end{array}$ & $\begin{array}{l}\text { Glass } \\
\text { (vol\%) }\end{array}$ & $\begin{array}{l}\text { Diatom } \\
\text { (vol\%) }\end{array}$ & $\begin{array}{l}\text { Radiolaria } \\
\text { (vol\%) }\end{array}$ & $\begin{array}{l}\text { Silicoflagellate } \\
\text { (vol\%) }\end{array}$ & $\begin{array}{c}\text { Foraminifer } \\
\text { (vol\%) }\end{array}$ & $\begin{array}{l}\text { Inorganic } \\
\text { carbonate } \\
\text { (vol\%) }\end{array}$ & $\begin{array}{c}\text { Organic } \\
\text { manter } \\
\text { (vol\%) }\end{array}$ & $\begin{array}{l}\text { Pyrite } \\
\text { (vol\%) }\end{array}$ & $\begin{array}{l}\text { Sponge } \\
\text { (vol\%) }\end{array}$ & $\begin{array}{l}\text { Total } \\
\text { maturer } \\
\text { (vol\%) }\end{array}$ & $\begin{array}{l}\text { Diatom } \\
\text { number } \\
\left(10^{7} / g\right)\end{array}$ & $\begin{array}{c}\text { Maximum } \\
\text { grain size }(\mu \mathrm{m})\end{array}$ \\
\hline $3 \mathrm{H}-5,103-105$ & 22.22 & color-banded & light & 92 & 27 & 55 & 2 & 8 & 0 & 0 & 0 & 0 & 2 & 5 & 1 & 100 & 0.340 & 116 \\
\hline $3 \mathrm{H}-6,14-16$ & 22.82 & laminated & dark & 73 & 40 & 40 & 0 & 3 & 0 & 0 & 0 & 2 & 3 & 10 & 2 & 100 & & 116 \\
\hline $3 \mathrm{H}-6,44-46$ & 23.11 & laminated & dark. & 73 & 38 & 41 & 0 & 0 & 0 & 0 & 0 & 3 & 3 & is & 0 & 100 & 0.040 & 224 \\
\hline $3 \mathrm{H}-6,73-75$ & 23.39 & laminated & dark & 73 & 35 & so & 0 & 1 & 0 & 0 & 0 & 3 & 4 & 7 & 0 & 100 & 0.570 & 266 \\
\hline $3 \mathrm{H}-6,103-105$ & 23.68 & color-banded & dark & 32 & 30 & 30 & 0 & 15 & 0 & 0 & 3 & 6 & 4 & 10 & 2 & 100 & 0.290 & 91 \\
\hline $3 \mathrm{H}-6,135-137$ & 23.99 & homiggeneous & light & ${ }_{15}^{8}$ & 26 & 50 & 2 & 0 & 0 & 0 & 6 & 7 & 2 & 7 & 0 & 100 & 0.010 & 133 \\
\hline $\begin{array}{l}3 \mathrm{H}-7.14-16 \\
4 \mathrm{H}-1.14-17\end{array}$ & $\begin{array}{l}24.27 \\
25.05\end{array}$ & $\begin{array}{l}\text { homogenencous } \\
\text { color banded }\end{array}$ & $\begin{array}{l}\text { faintly dark } \\
\text { light }\end{array}$ & $\begin{array}{l}35 \\
15\end{array}$ & $\begin{array}{l}30 \\
35\end{array}$ & $\begin{array}{l}40 \\
46\end{array}$ & 0 & 9 & 0 & ${ }_{0}^{0}$ & 5 & 6 & 2 & 6 & 2 & 100 & $\begin{array}{l}0.190 \\
0.570\end{array}$ & $\begin{array}{r}83 \\
108\end{array}$ \\
\hline $\begin{array}{l}4 \mathrm{HH}-1.14-17 \\
4 \mathrm{H}-1.44,46\end{array}$ & $\begin{array}{l}25.05 \\
25.34\end{array}$ & $\begin{array}{l}\text { colorob-banded } \\
\text { homogeneous }\end{array}$ & $\begin{array}{l}\text { light } \\
\text { light }\end{array}$ & $\begin{array}{r}15 \\
6\end{array}$ & $\begin{array}{l}35 \\
30\end{array}$ & $\begin{array}{l}46 \\
55\end{array}$ & $\frac{2}{2}$ & ${ }_{3}^{3}$ & $\begin{array}{l}0 \\
0\end{array}$ & $\begin{array}{l}0 \\
0\end{array}$ & $\begin{array}{l}1 \\
0\end{array}$ & $\begin{array}{l}4 \\
3\end{array}$ & $\begin{array}{l}1 \\
2\end{array}$ & ${ }_{4}^{7}$ & 1 & $\begin{array}{l}100 \\
100\end{array}$ & $\begin{array}{l}0.570 \\
1.100\end{array}$ & $\begin{array}{r}108 \\
83\end{array}$ \\
\hline $4 \mathrm{H}-1,74-76$ & 25.64 & color-banded & faintly dark & 44 & 20 & 40 & 0 & 20 & i & i & 0 & 5 & 2 & 8 & 3 & 100 & $\begin{array}{l}1.290 \\
1.290\end{array}$ & $\begin{array}{l}83 \\
83\end{array}$ \\
\hline $4 \mathrm{H}-\mathrm{I}, 102-104$ & 25.91 & color-banded & light & 10 & 25 & 36 & 1 & 25 & 0 & 0 & 0 & 3 & 1 & 6 & 3 & 100 & 1.930 & 75 \\
\hline $4 \mathrm{H}-1,135-137$ & $\begin{array}{l}26.23 \\
26.58\end{array}$ & $\begin{array}{l}\text { color-banded } \\
\text { ellorbanded }\end{array}$ & $\begin{array}{l}\text { light } \\
\text { dark }\end{array}$ & 20 & 27 & 35 & 0 & 25 & 1 & 3 & 0 & $\frac{1}{3}$ & 2 & 6 & 2 & 100 & 1.290 & 75 \\
\hline $\begin{array}{l}4 \mathrm{H}-2,20-22 \\
4 \mathrm{H}-2\end{array}$ & $\begin{array}{l}26.58 \\
2681\end{array}$ & $\begin{array}{l}\text { color-banded } \\
\text { color-banded }\end{array}$ & $\begin{array}{l}\text { dark } \\
\text { faint dark }\end{array}$ & 17 & 20 & $\begin{array}{l}23 \\
55\end{array}$ & 0 & 35 & ${ }_{0}^{2}$ & $\int_{0}^{1}$ & 2 & 3 & 3 & 8 & ${ }_{1}^{3}$ & ${ }_{100}^{100}$ & 5.140 & 83 \\
\hline 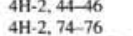 & $\begin{array}{l}27.11 \\
27.11\end{array}$ & $\begin{array}{c}\text { color-banded } \\
\text { laminated }\end{array}$ & $\begin{array}{l}\text { Faimlly yark } \\
\text { dark }\end{array}$ & $\begin{array}{r}7 \\
68\end{array}$ & $\begin{array}{l}27 \\
29\end{array}$ & $\begin{array}{l}55 \\
40\end{array}$ & ${ }_{0}^{2}$ & $\begin{array}{c}5 \\
15\end{array}$ & $\begin{array}{l}0 \\
0\end{array}$ & $\begin{array}{l}0 \\
0\end{array}$ & $\begin{array}{l}0 \\
0\end{array}$ & $\begin{array}{l}1 \\
2\end{array}$ & $\begin{array}{l}3 \\
6\end{array}$ & $\begin{array}{l}6 \\
7\end{array}$ & $\begin{array}{l}1 \\
1\end{array}$ & $\begin{array}{l}100 \\
100\end{array}$ & $\begin{array}{l}0.510 \\
1.710\end{array}$ & $\begin{array}{l}50 \\
66\end{array}$ \\
\hline $4 \mathrm{H}-2,103-105$ & 27.39 & laminated & dark & 68 & 35 & 28 & is & 8 & 0 & 0 & 0 & 3 & 4 & 5 & 2 & 100 & 1.710 & $\begin{array}{l}\infty \\
125\end{array}$ \\
\hline $4 \mathrm{H}-2,135-137$ & 27.71 & color-banded & faintly dark & 16 & 28 & 42 & 1 & 13 & 1 & 0 & 0 & 2 & 2 & 10 & 1 & 100 & 1.290 & 83 \\
\hline 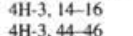 & $\begin{array}{l}27.99 \\
28.29\end{array}$ & $\begin{array}{l}\text { homogeneous } \\
\text { faminated }\end{array}$ & $\begin{array}{l}\text { light } \\
\text { durk } \\
\text { dint }\end{array}$ & ${ }_{23}^{17}$ & $\begin{array}{l}35 \\
30\end{array}$ & $\begin{array}{l}55 \\
35\end{array}$ & 2 & ${ }_{3}^{0}$ & $\begin{array}{l}0 \\
0\end{array}$ & 0 & 0 & 2 & 1 & 5 & 0 & 100 & 0.170 & 149 \\
\hline 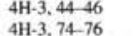 & $\begin{array}{l}28.29 \\
28.58\end{array}$ & $\begin{array}{l}\text { laminated } \\
\text { color-banded }\end{array}$ & $\begin{array}{l}\text { dark } \\
\text { faintly dark }\end{array}$ & ${ }_{3}^{23}$ & $\begin{array}{l}30 \\
34\end{array}$ & $\begin{array}{l}35 \\
50\end{array}$ & 10 & 3 & $\begin{array}{l}0 \\
0\end{array}$ & $\begin{array}{l}0 \\
0\end{array}$ & 8 & 5 & ${ }_{2}^{4}$ & $\begin{array}{l}4 \\
6\end{array}$ & 1 & 100 & $\begin{array}{l}0.540 \\
0.640\end{array}$ & $\begin{array}{l}141 \\
274\end{array}$ \\
\hline $\begin{array}{l}\begin{array}{l}4 \mathrm{H}-3,74,76 \\
4 \mathrm{H}-3,102-104\end{array}\end{array}$ & $\begin{array}{l}288.88 \\
28.85\end{array}$ & $\begin{array}{l}\text { coloro-banded } \\
\text { homogeneous }\end{array}$ & $\begin{array}{l}\text { faintly dark } \\
\text { light }\end{array}$ & $\begin{array}{c}3 \\
10\end{array}$ & $\begin{array}{l}34 \\
25\end{array}$ & $\begin{array}{l}50 \\
45\end{array}$ & $\frac{2}{3}$ & $\frac{2}{5}$ & $\begin{array}{l}0 \\
0\end{array}$ & $\begin{array}{l}0 \\
0\end{array}$ & ${ }_{5}^{2}$ & ${ }_{10}^{2}$ & ${ }_{0}^{2}$ & $\begin{array}{l}6 \\
7\end{array}$ & $\begin{array}{l}0 \\
0\end{array}$ & $\begin{array}{l}100 \\
100\end{array}$ & $\begin{array}{l}0.640 \\
1.290\end{array}$ & $\begin{array}{r}274 \\
83\end{array}$ \\
\hline $4 \mathrm{H} \cdot 3,135-137$ & 29.18 & laminated & faintly dark & 13 & 20 & 35 & 0 & 7 & i & 0 & 8 & 10 & 8 & 10 & $\begin{array}{l}0 \\
1\end{array}$ & $\begin{array}{l}100 \\
100\end{array}$ & $\begin{array}{l}1.290 \\
1.710\end{array}$ & $\begin{array}{l}83 \\
66\end{array}$ \\
\hline $4 \mathrm{H}-4,13-15$ & 29.45 & homogeneous & light & 23 & 25 & 57 & i & 5 & 0 & 0 & i & 4 & 0 & 6 & i & 100 & 0.960 & 83 \\
\hline $4 \mathrm{H}-4,44,46$ & 29.76 & homogeneous & light & 39 & 25 & 35 & 0 & 27 & 1 & 2 & 0 & 1 & i & 6 & 2 & 100 & 1.290 & 100 \\
\hline $\begin{array}{l}4 \mathrm{H}-4,74-76 \\
4 \mathrm{H}-4,102-104\end{array}$ & $\begin{array}{l}30.05 \\
3033\end{array}$ & homogeneous & light & 39 & $\begin{array}{l}35 \\
17\end{array}$ & $\begin{array}{r}53 \\
40\end{array}$ & 2 & 4 & 0 & 0 & 0 & 2 & 0 & 3 & i & 100 & 0.200 & 66 \\
\hline 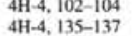 & $\begin{array}{l}30.33 \\
30.65\end{array}$ & $\begin{array}{l}\text { laminated } \\
\text { laminated }\end{array}$ & $\begin{array}{l}\text { dark } \\
\text { dark }\end{array}$ & $\begin{array}{r}4 \\
30\end{array}$ & $\begin{array}{l}17 \\
10\end{array}$ & ${ }_{24}^{40}$ & $\begin{array}{l}0 \\
1\end{array}$ & $\begin{array}{l}10 \\
20\end{array}$ & $\begin{array}{l}0 \\
0\end{array}$ & 0 & ${ }_{20}^{7}$ & 8 & 10 & 8 & 0 & 100 & 1.540 & 66 \\
\hline $4 \mathrm{H}-5,14-16$ & 30.94 & color-banded & $\begin{array}{l}\text { fainty } \\
\text { farntly dark }\end{array}$ & $\begin{array}{l}30 \\
22\end{array}$ & 30 & 48 & 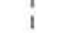 & $\begin{array}{l}20 \\
12\end{array}$ & $\begin{array}{l}0 \\
0\end{array}$ & $\begin{array}{l}0 \\
0\end{array}$ & $\begin{array}{r}20 \\
0\end{array}$ & 15 & 6 & 4 & 0 & 100 & 2.060 & 50 \\
\hline $4 \mathrm{H}-5,43-45$ & 31.22 & homogeneous & light & 20 & 15 & 37 & i & $\frac{12}{3}$ & 0 & $\begin{array}{l}0 \\
0\end{array}$ & 10 & $\begin{array}{c}1 \\
30\end{array}$ & $\begin{array}{l}1 \\
0\end{array}$ & 6 & i & 100 & $\begin{array}{l}1.290 \\
0770\end{array}$ & ${ }_{125}^{83}$ \\
\hline $4 \mathrm{H}-5,71-73$ & 31.49 & laminated & dark & II & 35 & 40 & 2 & 10 & i & 0 & 0 & 1 & 2 & 8 & 1 & 100 & 1.100 & $\begin{array}{r}125 \\
75\end{array}$ \\
\hline $4 \mathrm{H}-5,102-104$ & $\begin{array}{l}31.80 \\
30.12\end{array}$ & color-banded & light & 59 & 30 & 41 & 2 & 20 & 0 & 0 & 0 & i & $i$ & 5 & 0 & 100 & 1.030 & 75 \\
\hline $4 \mathrm{H}-5,135-137$ & 32.12 & & light & 59 & 25 & 44 & 1 & 20 & 1 & 1 & 0 & 0 & 1 & 6 & 1 & 100 & 0.860 & 58 \\
\hline $4 \mathrm{H}-6,14-16$ & 32.41 & homogeneous & light & 39 & 25 & 55 & i & 10 & i & 0 & 0 & i & i & 5 & i & 100 & 0.510 & 91 \\
\hline $4 \mathrm{H}-6,44-46$ & 32.70 & laminated & dark & 106 & 40 & 44 & 1 & 2 & 0 & 0 & 0 & 1 & 2 & 10 & 0 & 100 & 0.510 & 141 \\
\hline 4H-6.73-75 & 32.99 & laminated & dark & 106 & 40 & 39 & 1 & 2 & 0 & 0 & 0 & 1 & 4 & II & 2 & 100 & 0.290 & $\begin{array}{l}183 \\
158\end{array}$ \\
\hline $\begin{array}{l}4 \mathrm{H}-6,102-104 \\
4 \mathrm{H}-6\end{array}$ & $\begin{array}{l}33.27 \\
3359\end{array}$ & $\begin{array}{l}\begin{array}{c}\text { laminated } \\
\text { laminated }\end{array} \\
\text { and }\end{array}$ & dark & 106 & 35 & 38 & 0 & 7 & 0 & 0 & 0 & 0 & 3 & 15 & 2 & 100 & 1.290 & 158 \\
\hline $\begin{array}{l}4 \mathrm{H}-6.6,135-137 \\
5 \mathrm{H}-1,14-16\end{array}$ & $\begin{array}{l}333.59 \\
34.54\end{array}$ & $\begin{array}{l}\text { laminated } \\
\text { homogeneous }\end{array}$ & $\begin{array}{l}\text { dark } \\
\text { light }\end{array}$ & $\begin{array}{r}106 \\
31\end{array}$ & $\begin{array}{l}24 \\
20\end{array}$ & $\begin{array}{l}25 \\
70\end{array}$ & $\begin{array}{l}0 \\
2\end{array}$ & $\frac{2}{3}$ & $\begin{array}{l}0 \\
0 \\
0\end{array}$ & $\begin{array}{l}0 \\
0\end{array}$ & $\begin{array}{c}15 \\
0\end{array}$ & $\begin{array}{l}15 \\
0\end{array}$ & ${ }_{0}^{3}$ & $\begin{array}{r}15 \\
4\end{array}$ & i & $\begin{array}{l}100 \\
100\end{array}$ & $\begin{array}{l}0.200 \\
0.450\end{array}$ & $\begin{array}{l}100 \\
133 \\
133\end{array}$ \\
\hline $5 \mathrm{H}-1,44,46$ & 34.83 & laminated & $\begin{array}{l}\text { dignt } \\
\text { dark }\end{array}$ & $\begin{array}{l}31 \\
8\end{array}$ & 20 & $\begin{array}{l}70 \\
67\end{array}$ & 1 & 1 & 0 & 0 & 0 & 2 & 4 & 4 & 1 & 100 & $\begin{array}{l}0.450 \\
0.110\end{array}$ & $\begin{array}{l}133 \\
149\end{array}$ \\
\hline $5 \mathrm{H}-1,74-76$ & 35.12 & bioturbated & light & 28 & 25 & 61 & 1 & i & 0 & 0 & 0 & 3 & 2 & 7 & 0 & 100 & 0.120 & 100 \\
\hline $5 \mathrm{H}-1,103-105$ & 35.41 & laminated & dark & 27 & 40 & 40 & 0 & 0 & 0 & 0 & 0 & 2 & 3 & 15 & 0 & 100 & 0.010 & 166 \\
\hline $5 \mathrm{H}-1,132-134$ & 35.69 & color-banded & dark & 2 & 35 & 45 & 1 & 0 & 0 & 0 & 2 & 7 & 3 & 7 & 0 & 100 & 0.010 & 183 \\
\hline $5 \mathrm{H}-2,17-19$ & $\begin{array}{l}36.02 \\
36.28\end{array}$ & $\begin{array}{l}\text { bioturbated } \\
\text { colorsbanded }\end{array}$ & $\begin{array}{l}\text { light } \\
\text { liegt }\end{array}$ & 8 & 35 & 48 & 2 & 5 & 0 & ${ }_{0}^{0}$ & 0 & 1 & 1 & 6 & 2 & 100 & 0.860 & 125 \\
\hline $\begin{array}{l}5 \mathrm{H}-2,2,44-46 \\
5 \mathrm{H}-2,74-76\end{array}$ & $\begin{array}{l}36.28 \\
36.57\end{array}$ & $\begin{array}{l}\text { colorrb-banded } \\
\text { color-banded }\end{array}$ & $\begin{array}{l}\text { light } \\
\text { light }\end{array}$ & $\begin{array}{l}14 \\
12\end{array}$ & $\begin{array}{l}40 \\
37\end{array}$ & $\begin{array}{l}46 \\
50\end{array}$ & 2 & $\begin{array}{l}0 \\
0\end{array}$ & $\begin{array}{l}0 \\
0\end{array}$ & $\begin{array}{l}0 \\
0\end{array}$ & $\begin{array}{l}0 \\
0\end{array}$ & ${ }_{3}^{4}$ & $\frac{2}{3}$ & ${ }_{5}^{6}$ & $\begin{array}{l}0 \\
0\end{array}$ & $\begin{array}{l}100 \\
100\end{array}$ & 0.020 & 125 \\
\hline SH-2, 103-105 & 36.86 & color-banded & $\begin{array}{l}\text { light } \\
\text { light }\end{array}$ & $\begin{array}{l}12 \\
8\end{array}$ & $\begin{array}{l}37 \\
24\end{array}$ & $\begin{array}{l}50 \\
45\end{array}$ & $\frac{2}{3}$ & $\begin{array}{l}0 \\
0 \\
0\end{array}$ & $\begin{array}{l}0 \\
0\end{array}$ & $\begin{array}{l}0 \\
0\end{array}$ & ${ }_{7}^{0}$ & $10^{3}$ & 3 & 8 & ${ }_{0}^{0}$ & 100 & $\begin{array}{l}0.030 \\
0.140\end{array}$ & $\begin{array}{l}166 \\
141\end{array}$ \\
\hline $5 \mathrm{H}-2,132-134$ & 37.14 & & $\begin{array}{l}\text { dark } \\
\text { dark }\end{array}$ & 7 & 30 & 40 & 3 & 3 & 0 & 0 & 0 & 2 & 3 & $10^{\circ}$ & i & 100 & 1.100 & $\begin{array}{l}141 \\
100\end{array}$ \\
\hline SH-3, 14-16 & 37.44 & homogeneous & tight & 15 & 35 & 55 & 4 & 0 & 0 & 0 & 0 & 2 & 1 & 3 & 0 & 100 & 0.090 & $\begin{array}{l}100 \\
149\end{array}$ \\
\hline $5 \mathrm{H}-3,44-46$ & 37.73 & color-banded & dark & 16 & 23 & 60 & 2 & 5 & 0 & 0 & 0 & $i$ & 2 & 5 & 2 & 100 & 0.200 & 83 \\
\hline $5 \mathrm{H}-3,74-76$ & 38.02 & color-banded & faintly dark & 18 & 25 & 57 & $i$ & 7 & $i$ & 0 & 0 & $i$ & i & 5 & 2 & 100 & 0.960 & 75 \\
\hline $5 \mathrm{H}-3,102-104$ & 38.30 & homogeneous & light & 73 & 25 & 65 & 2 & 4 & i & 0 & 0 & 0 & 0 & 2 & I & 100 & 0.700 & 83 \\
\hline $5 \mathrm{H}-3,132-134$ & 38.59 & homogeneous & light & 73 & 25 & 63 & 1 & 0 & 0 & 0 & 0 & 0 & 1 & 10 & 0 & 100 & 0.060 & 208 \\
\hline $5 \mathrm{H}-4,14-16$ & 38.89 & laminated & dark & 5 & 40 & 45 & 1 & 0 & 0 & 0 & 0 & 4 & 3 & 7 & 0 & 100 & 0.040 & 216 \\
\hline $5 \mathrm{H}-4,43-45$ & 39.17 & color-banded & light & $?$ & & 55 & 1 & 0 & 0 & 0 & 0 & 3 & 1 & & 0 & 100 & 0.030 & 183 \\
\hline & $\begin{array}{l}39.47 \\
39.75\end{array}$ & homogeneous & $\begin{array}{l}\text { light } \\
\text { lankty }\end{array}$ & 8 & 35 & 44 & 0 & 0 & ${ }_{0}^{0}$ & ${ }_{0}^{0}$ & 2 & 8 & 1 & 10 & 0 & 100 & 0.330 & 208 \\
\hline $\begin{array}{l}5 \mathrm{H}-4,103-105 \\
5 \mathrm{H}-4,131-133\end{array}$ & $\begin{array}{l}39.75 \\
4003\end{array}$ & $\begin{array}{l}\text { color-banded } \\
\text { color-banded }\end{array}$ & $\begin{array}{l}\text { faintly dark } \\
\text { tight }\end{array}$ & $\begin{array}{r}28 \\
7\end{array}$ & $\begin{array}{l}25 \\
30\end{array}$ & $\begin{array}{l}50 \\
53\end{array}$ & 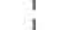 & 15 & 0 & $\begin{array}{l}0 \\
0\end{array}$ & 0 & 1 & 2 & 5 & 1 & 100 & 1.290 & 83 \\
\hline $\begin{array}{l}5 \mathrm{H}-4,131-133 \\
5 \mathrm{H}-5,11-13\end{array}$ & $\begin{array}{l}40.03 \\
40.32\end{array}$ & $\begin{array}{l}\text { color-binded } \\
\text { color-banded }\end{array}$ & 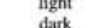 & 4 & $\begin{array}{l}30 \\
15\end{array}$ & $\begin{array}{l}33 \\
40\end{array}$ & 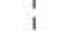 & 3 & ${ }_{0}^{0}$ & 0 & 5 & 15 & $10^{3}$ & 10 & 1 & & 0.440 & 83 \\
\hline $5 \mathrm{H}-5,43-45$ & 40.62 & bioturbated & $\begin{array}{l}\text { light } \\
\text { ling }\end{array}$ & 33 & 30 & 53 & $\frac{1}{2}$ & 4 & 0 & 0 & i & 5 & 1 & 3 & 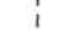 & $\begin{array}{l}100 \\
100\end{array}$ & $\begin{array}{l}2.570 \\
.290\end{array}$ & 83 \\
\hline $5 \mathrm{H}-5,74-76$ & 40.92 & bioturbated & light & 30 & 25 & 57 & 2 & 5 & 0 & 0 & 0 & 5 & $i$ & 4 & $i$ & 100 & $\begin{array}{l}1.290 \\
2.570\end{array}$ & $\begin{array}{l}100 \\
133\end{array}$ \\
\hline SH-5, 103-105 & 41.20 & bioturbated & light & 106 & 30 & 53 & 2 & 7 & $i$ & 0 & 0 & 2 & i & 3 & $\mathrm{i}$ & 100 & 0.640 & 83 \\
\hline $5 \mathrm{H}-5,131-133$ & 41.48 & bioturbated & light & 106 & 5 & 20 & 1 & 2 & 0 & 0 & 10 & 60 & 0 & 2 & 0 & 100 & 3.860 & 58 \\
\hline $5 \mathrm{H}-6,14-16$ & 41.79 & homogeneous & light & 106 & 14 & 50 & 2 & 5 & 0 & 0 & 1 & 25 & 0 & 2 & 1 & 100 & 3.430 & 125 \\
\hline $5 \mathrm{H}-6,44-46$ & 42.08 & laminated & dark & 13 & 30 & 23. & 1 & 30 & 0 & 0 & 0 & 1 & 6 & 7 & 2 & 100 & 2.570 & 581 \\
\hline SH-6, 81-83 & $\begin{array}{l}42,44 \\
4264\end{array}$ & laminated & dark & 3 & 40 & $\begin{array}{l}36 \\
36 \\
28\end{array}$ & 0 & $\begin{array}{l}0 \\
5\end{array}$ & 0 & 0 & 0 & $20^{2}$ & 2 & 20 & 0 & 100 & $\begin{array}{l}0.030 \\
0200\end{array}$ & 249 \\
\hline $\begin{array}{l}5 \mathrm{H}-6,102-104 \\
5 \mathrm{H}-6.131-133\end{array}$ & $\begin{array}{l}42.64 \\
42.93\end{array}$ & $\begin{array}{c}\text { laminated } \\
\text { homogeneous }\end{array}$ & $\begin{array}{l}\text { dark } \\
\text { liehs }\end{array}$ & $\begin{array}{l}33 \\
14\end{array}$ & $\begin{array}{l}25 \\
45\end{array}$ & $\begin{array}{l}28 \\
40\end{array}$ & 0 & $\begin{array}{l}5 \\
0\end{array}$ & $0_{0}^{0}$ & $\begin{array}{l}0 \\
0\end{array}$ & $3_{0}^{3}$ & $\frac{20}{4}$ & 3 & 15 & 1 & $\begin{array}{l}100 \\
100\end{array}$ & $\begin{array}{l}0.290 \\
0.030\end{array}$ & $\begin{array}{l}174 \\
1791\end{array}$ \\
\hline $5 \mathrm{H}-7,14-16$ & $\begin{array}{l}42.93 \\
43.24\end{array}$ & $\begin{array}{l}\text { nomogenecous } \\
\text { laminated }\end{array}$ & $\begin{array}{l}\text { Mignt } \\
\text { faintly dark }\end{array}$ & $\begin{aligned} 14 \\
8\end{aligned}$ & $\begin{array}{l}45 \\
25\end{array}$ & $\begin{array}{l}40 \\
18\end{array}$ & 1 & 20 & 1 & 0 & 2 & 20 & 3 & 7 & 3 & 100 & $\begin{array}{l}0.030 \\
1.540\end{array}$ & $\begin{array}{l}291 \\
125\end{array}$ \\
\hline $5 \mathrm{H}-7,43-45$ & 43.52 & color-banded & light & 4 & 35 & 40 & 0 & 0 & 0 & 0 & 0 & 12 & 3 & 10 & 0 & 100 & 0.260 & 166 \\
\hline $6 \mathrm{H}-1,13-15$ & 44.03 & homogeneous & light & 20 & 40 & 47 & 1 & 1 & 0 & 0 & 0 & 1 & 1 & 8 & 1 & 100 & 0.420 & 166 \\
\hline $6 \mathrm{H}-1.43-45$ & 44.32 & homogeneous & light & $3 !$ & 15 & 65 & 1 & 7 & 1 & 0 & 0 & 1 & 1 & 7 & 2 & 100 & 1.100 & 58 \\
\hline $6 \mathrm{H}-1,74-76$ & 44.62 & homogeneous & light & 54 & 25 & 55 & 1 & 10 & 0 & 0 & 0 & 0 & 2 & 5 & 2 & 100 & 0.460 & 108 \\
\hline $6 \mathrm{H}-1,102-104$ & 44.89 & color-banded & light & 54 & 40 & 32 & 2 & 15 & ${ }_{0}^{0}$ & ${ }_{0}^{0}$ & 0 & 9 & $\frac{2}{2}$ & $\begin{array}{l}5 \\
8\end{array}$ & 4 & 100 & 0.150 & 116 \\
\hline $6 \mathrm{H}-1,132-134$ & $\begin{array}{l}45.18 \\
45.48\end{array}$ & $\begin{array}{l}\text { laminated } \\
\text { homoeneous }\end{array}$ & $\begin{array}{l}\text { dark } \\
\text { liaphs }\end{array}$ & $\underset{9}{11}$ & $\begin{array}{l}27 \\
40\end{array}$ & $\begin{array}{l}50 \\
{ }_{43}\end{array}$ & 0 & ${ }_{0}^{3}$ & ${ }_{0}^{0}$ & $\begin{array}{l}0 \\
0\end{array}$ & $\begin{array}{l}1 \\
0\end{array}$ & ${ }_{3}^{7}$ & 2 & 8 & 2 & 100 & 1.140 & $\begin{array}{l}266 \\
374\end{array}$ \\
\hline $\begin{array}{l}6 \mathrm{H}-2.13-15 \\
6 \mathrm{H}-2,43-45\end{array}$ & $\begin{array}{l}45.48 \\
45.76\end{array}$ & $\begin{array}{l}\text { homogenceus } \\
\text { color-banded }\end{array}$ & faintly dark & 12 & 25 & $\begin{array}{l}43 \\
55\end{array}$ & 2 & 10 & 0 & 0 & 0 & $i$ & i & 4 & $\begin{array}{l}1 \\
2\end{array}$ & $\begin{array}{l}100 \\
100\end{array}$ & $\begin{array}{l}0.410 \\
1080\end{array}$ & $\begin{array}{r}374 \\
58\end{array}$ \\
\hline
\end{tabular}


225 at Site 795, and 50 to 230 at Site 797. The amplitude in gray value variation is smaller by 65 at Site 795 compared to Sites 794 and 797. The frequency of fluctuation in gray value varies from $0.5 \mathrm{cycle} / \mathrm{m}$ to 5 cycles $/ \mathrm{m}$. There appears to be a quasi-cyclic change in the frequency with a wavelength of several meters.

In order to enable comparisons to be drawn between gray values and other analytical data, we estimated the gray value for each sample by interpolating the measured gray values just above and below the sampling position to the exact sampling position. Because the gray value was measured at $2-\mathrm{cm}$ intervals, the distance to the closest measurement points from the sampling position is less than $1 \mathrm{~cm}$. The estimated values are included in Table 2.

For samples from Site 797, gray values of dark, faintly dark, and light layers range from 136 to 218,130 to 20.4 , and 50 to 150 , respectively. The gray values of dark and faintly dark layers overlap considerably, whereas the gray values of faintly dark and light layers are relatively well separated at approximately 140 .

\section{Correlation of Dark and Light Layers}

The excellent recovery and preservation of cores from Sites 794, 795, and 797 has enabled us to attempt correlation of the dark and light layers among the three sites. Visual correlation of vertical profiles of gray value above the Brunhes/Matuyama boundaries was conducted (Fig. 4), and is cross checked and supplemented by visual inspection of core photographs (Pls. 1-5). Correlation of thick $(>30 \mathrm{~cm})$ dark layers, which are recognized as wide peaks on the gray value profiles, is relatively straightforward. They are alphabetically labeled from A to $\mathrm{M}$ in descending order (Figs. 2 and 4, Pls. 1-5). In addition to the thick dark layers, correlation of thinner dark layers was also carried out on core photographs (Pls. 1-5). The correlation of dark layers among the three sites is presented in Figure 2.

Most dark layers within the upper Quaternary sediments are correlatable among the three sites. However, the correlation of dark layers among the sites is somewhat ambiguous within the stratigraphic intervals between the dark layers $\mathrm{C}$ and $\mathrm{F}$ at Site 794 and between the dark layers $\mathrm{F}$ and $\mathrm{G}$ at Site 795. Close inspection of core photographs within these intervals suggests a lack of sediment at core breaks. A lack of sediment equivalent to approximately $1.5 \mathrm{~m}$ thick is estimated at the core break between Cores $127-794 \mathrm{~A}-1 \mathrm{H}$ and $127-794 \mathrm{~A}-2 \mathrm{H}$ (between the dark layers $\mathrm{C}$ and F). A lack of sediment equivalent to approximately $50 \mathrm{~cm}$ is estimated at the core break between Cores 127-797B-4H and 127797B-5H. A lack of sediment is also suggested at the core break between Cores $127-795 \mathrm{~A}-2 \mathrm{H}$ and 127-795A-3H (between the dark layers F and G) although not conclusive.

The correlation of the dark layers illustrated in Figure 2 is consistent with the chronologic framework. Namely, dark layers B and M show a parallel relationship with marker ash layers, Aso- 4 and $\mathrm{T}_{1}$, and the dark layer $\mathrm{M}$ shows a nearly parallel relationship with the Brunhes/Matuyama paleomagnetic boundary (Pl. 1, Fig. 2, Pl. 5, Figs. 1 and 2). Correlation of the dark layers is also consistent with the LO of $R$. curvirostris and the LO of $P$. lacunosa.

The parallel relationship between marker ash layers or paleomagnetic boundaries and dark layers suggests that the deposition of dark layers in the Japan Sea during the late Quaternary was a nearly synchronous and basinwide phenomenon. Synchronism of the deposition is estimated to within 300 years for dark layers B and M based on the difference in calculated time intervals between the dark layer and the adjacent marker ash layer among the three sites. The time intervals are calculated as the vertical distance between the two layers divided by the average sedimentation rate at each site.

\section{Microscopic Observation}

The results of smear slide analysis for samples from Site 797 are listed in Table 1. The sediments are composed of clay- to silt-size detrital material with various amounts of siliceous and calcareous biogenic components, organic matter, and pyrite.
Detrital silt consists dominantly of monocrystalline quartz and plagioclase. Polycrystalline quartz of possible metamorphic origin and greenish amphibole is present in small amounts in some samples. The composition of the clay-size detrital component is difficult to identify under the optical microscope and will not be discussed. The content of detrital silt is generally between 20 and $50 \mathrm{vol} \%$, whereas that of detrital clay is generally between 20 and $80 \mathrm{vol} \%$.

Volcanic glass shards and pumice grains, fresh or altered, are minor constituents. They are dominantly of silt-size.

Inorganic carbonate is common and present as clay- and silt-size grains. The inorganic carbonate content is generally below $20 \mathrm{vol} \%$ though it does reach as high as $60 \mathrm{vol} \%$ in one sample. Although it is irregular in shape, we did not include inorganic carbonate in the detrital clay and silt category but treated it independently, since it is possible that some of the clay-size carbonate grains are fragments of calcareous fossils which were fragmented after burial or during the preparation of smear slides.

The biogenic siliceous component consists dominantly of diatoms, with minor amounts of sponge spicules, radiolarians, and silicoflagellates. Fragments of these siliceous fossils are also present as clay-size particles. The content of biogenic silica is variable and may reach as much as $30 \mathrm{vol} \%$.

The biogenic calcareous component consists dominantly of planktonic foraminifers with minor to moderate amounts of benthic foraminifers and nannofossils. Nannofossils, although present in some samples, were not quantified. The content of the biogenic calcareous component is generally low in most samples but may reach as high as $20 \mathrm{vol} \%$ in several samples.

Pyrite is a common diagenetic component. It occurs as framboid, large $(>500 \mu \mathrm{m})$ cubic crystals, and tiny $(<2 \mu \mathrm{m})$ particles. Its amount is variable and may comprise as much as $20 \mathrm{vol} \%$.

Organic matter is also common in most samples. It is present as tiny, brownish amorphous pigments and carbonaceous plant fragments. Its amount is generally small, but can reach as high as $10 \mathrm{vol} \%$.

The maximum diameter of the detrital quartz and plagioclase grains ranges from 50 to $580 \mu \mathrm{m}$. Silt-size quartz grains, either monocrystalline or polycrystalline, are rounded to angular, whereas plagioclase grains are generally angular.

The relationship between the biogenic component and the maximum diameter of the detrital grains (Fig. 5) suggests that sediments with abundant biogenic materials (mostly diatoms and foraminifers) tend to be finer grained than the sediments with few biogenic materials. On the other hand, the maximum grain size, the detrital silt content, and the content of biogenic materials do not necessarily change drastically between the dark, faintly dark and light layers, and both the dark, faintly dark, or light layers can be rich in biogenic materials or silt grains.

\section{Mineralogy}

The result of the XRD analysis is shown in Table 2. The upper Quaternary fine-grained sediments at Site 797 analyzed in this study are composed mainly of detrital minerals, such as quartz, plagioclase, illite, kaolinite plus chlorite, and possibly smectite; biogenic minerals such as opal-A and calcite; and diagenetic minerals, such as pyrite.

Figure 6 shows the relationship between the peak heights of the previously mentioned detrital minerals. Quartz vs. plagioclase (Fig. 6A), and smectite vs. kaolinite plus chlorite (Fig. 6B) have a higher positive correlation $\left(r^{2}=0.67\right)$ with approximately zero intercept, suggesting that the mineral pairs keep nearly constant ratios. Quartz and illite also show a positive correlation with approximately zero intercept (Fig. 6C). However, the correlation is lower $\left(r^{2}=0.53\right)$, suggesting that the ratio between the quartz and plagioclase pair vs. the illite and kaolinite plus chlorite pair is not constant within the detrital component.

We believe that the smectite is also of detrital origin. A diagenetic or hydrothermal origin is unlikely considering the young age $(<\mathrm{lMa})$, shallow burial depth $(<100 \mathrm{~m})$, and low temperature $\left(<10^{\circ} \mathrm{C}\right)$ of the 
Gray value

Gray value

Gray value

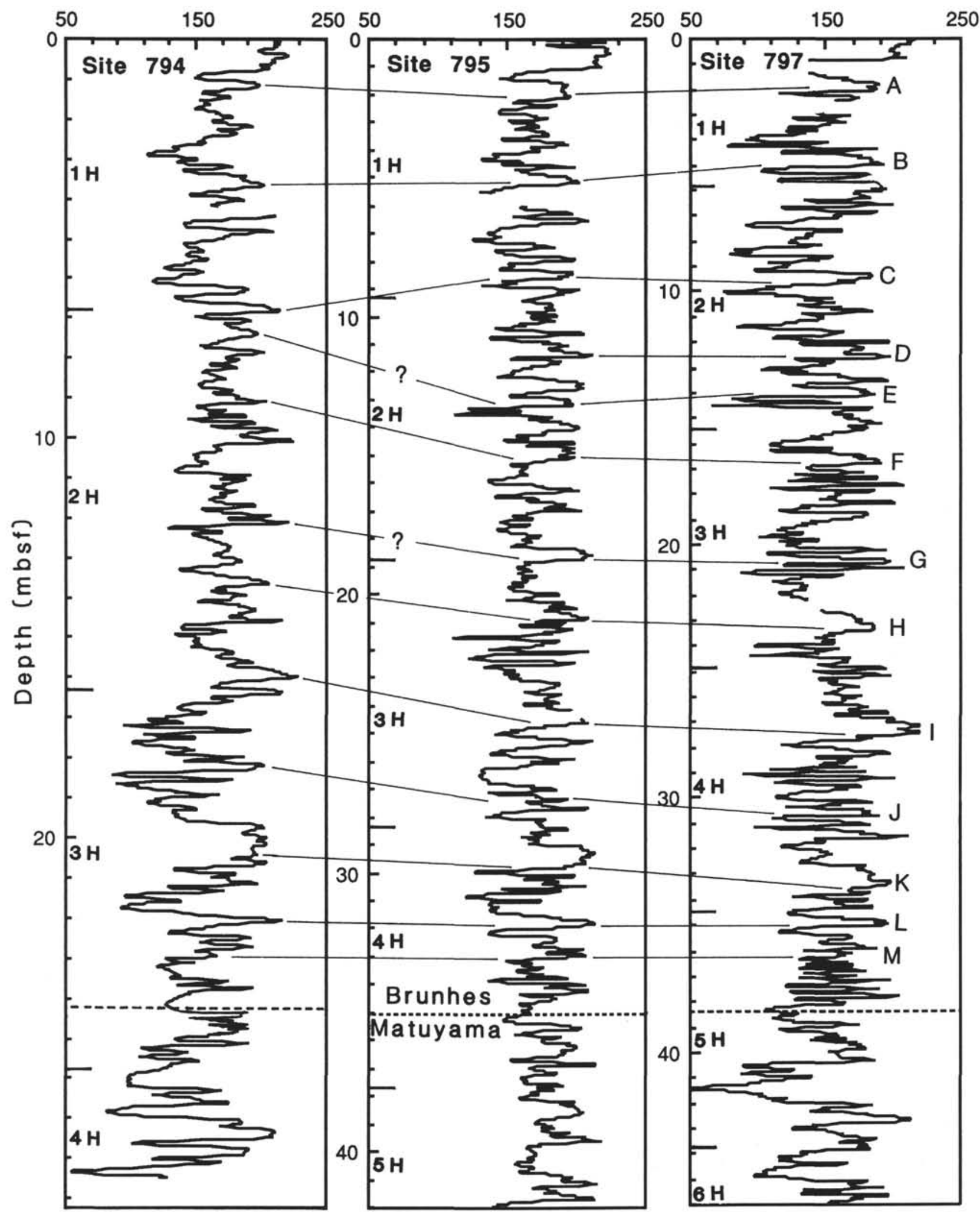

Figure 4. Vertical profiles of gray values for the upper Quaternary sediments from Sites 794, 795, and 797 and their correlation. The labels A through M correspond to those in Figure 2. 
Table 2. X-ray diffraction, carbon, and sulfur analyses and the estimated gray value.

\begin{tabular}{|c|c|c|c|c|c|c|c|c|c|c|c|c|c|}
\hline $\begin{array}{l}\text { Core, section, } \\
\text { interval }(\mathrm{cm})\end{array}$ & $\begin{array}{l}\text { Depth } \\
\text { (mbsf) }\end{array}$ & $\begin{array}{c}\mathrm{C}_{\mathrm{org}} \\
(\mathrm{wt} \%)\end{array}$ & $\begin{array}{l}\mathrm{C}_{\mathrm{carb}} \\
(\mathrm{wt} \%)\end{array}$ & $\underset{(w t \%)}{\mathrm{S}_{\text {tot }}}$ & $\begin{array}{l}\text { Gray } \\
\text { value }\end{array}$ & $\begin{array}{c}\text { Quartz } \\
\text { (cps/10) }\end{array}$ & $\begin{array}{l}\text { Plagioclase } \\
\text { (cps/10) }\end{array}$ & $\begin{array}{c}\text { Illite } \\
(\mathrm{cps} / 10)\end{array}$ & $\begin{array}{l}\text { Kaolinite + chlorite } \\
(\mathrm{cps} / 10)\end{array}$ & $\begin{array}{l}\text { Smectite } \\
(\mathrm{cps} / 10)\end{array}$ & $\begin{array}{l}\text { Calcite } \\
(\mathrm{cps} / 10)\end{array}$ & $\begin{array}{c}\text { Pyrite } \\
(\mathrm{cps} / 10)\end{array}$ & $\begin{array}{r}\text { Opal-A } \\
(\mathrm{cps} / 10)\end{array}$ \\
\hline \multicolumn{14}{|l|}{$127-797 \mathrm{~B}-$} \\
\hline $1 \mathrm{H}-1,13-15$ & 0.13 & 0.78 & 0.07 & 0.02 & 213 & 88.0 & 17.0 & 11.0 & 8.5 & 2.0 & 0.0 & 0.0 & 4.0 \\
\hline $1 \mathrm{H}-1,44-46$ & 0.43 & 1.10 & 0.08 & 0.00 & 194 & 71.0 & 15.0 & 6.0 & 6.0 & 4.0 & 0.0 & 0.0 & 2.5 \\
\hline IH-1,74-76 & 0.71 & 2.08 & 0.09 & 0.03 & 196 & 72.5 & 16.0 & 6.0 & 7.0 & 3.0 & 0.0 & 1.0 & 3.5 \\
\hline $1 \mathrm{H}-1,134-136$ & 1.29 & 1.46 & 1.10 & 2.74 & 136 & 95.0 & 17.5 & 10.5 & 12.0 & 0.0 & 19.5 & 10.0 & 2.8 \\
\hline $1 \mathrm{H}-2,13-15$ & 1.56 & 1.12 & 0.89 & 2.38 & 144 & 102.5 & 15.5 & 11.0 & 8.5 & 1.5 & 13.0 & 11.5 & 1.5 \\
\hline $1 \mathrm{H}-2,44-46$ & 1.86 & 1.06 & 0.66 & 2.92 & 194 & 100.5 & 23.0 & 14.0 & 14.0 & 0.0 & 10.5 & 11.5 & 0.8 \\
\hline $1 \mathrm{H}-2,74-76$ & 2.14 & 0.53 & 0.18 & 0.59 & 139 & 120.0 & 23.0 & 10.0 & 12.0 & 0.0 & 0.0 & 3.5 & 2.3 \\
\hline $1 \mathrm{H}-2,104-106$ & 2.43 & 0.96 & 0.18 & 0.16 & 164 & 103.0 & 18.5 & 10.0 & 11.0 & 3.0 & 0.0 & 0.0 & 2.5 \\
\hline IH-3, 13-15 & 2.99 & 0.49 & 0.19 & 0.02 & 125 & 121.0 & 30.0 & 20.0 & 19.0 & 0.0 & 2.0 & 0.0 & 2.5 \\
\hline IH $-3,44-46$ & 3.29 & 1.60 & 0.15 & 0.12 & 164 & 111.5 & 24.0 & 12.5 & 15.0 & 2.5 & 0.0 & 0.0 & 2.5 \\
\hline $1 \mathrm{H}-3,74-76$ & 3.57 & 0.35 & 0.17 & 0.01 & 122 & 123.5 & 23.5 & 11.5 & 16.5 & 0.0 & 0.0 & 0.0 & 1.3 \\
\hline $\mathrm{IH}-3,104-106$ & 3,86 & 0.51 & 0.16 & 0.00 & 120 & 112.0 & 23.0 & 11.0 & 10.5 & 0.0 & 0.0 & 0.0 & 2.3 \\
\hline $1 \mathrm{H}-3,134-136$ & 4.14 & 3.37 & 0.25 & 3.55 & 162 & 88.0 & 17.5 & 10.5 & 8.5 & 3.0 & 3.0 & 13.0 & 3.0 \\
\hline $1 \mathrm{H}-4,13-15$ & 4.42 & 4.32 & 0.12 & 3.09 & 189 & 82.5 & 18.0 & 7.0 & 9.0 & 1.5 & 0.0 & 14.5 & 3.3 \\
\hline IH- $4,44-46$ & 4.71 & 1.82 & 0.09 & 0.42 & 165 & 92.5 & 20.0 & 11.0 & 11.5 & 0.0 & 0.0 & 0.0 & 2.0 \\
\hline $1 \mathrm{H}-4,71-73$ & 4.97 & 3.43 & 0.10 & 1.67 & 193 & 71.5 & 15.0 & 6.5 & 6.5 & 4.0 & 0.0 & 8.5 & 3.3 \\
\hline $1 \mathrm{H}-4,104-106$ & 5.29 & 0.38 & 0.77 & 0.08 & 103 & 105.5 & 23.5 & 12.0 & 11.5 & 1.5 & 16.5 & 1.5 & 2.8 \\
\hline $2 \mathrm{H}-1,14-16$ & 6.05 & 0.76 & 0.14 & 0.90 & 204 & 102.0 & 24.0 & 12.5 & 11.5 & 0.0 & 0.0 & 4.5 & 1.8 \\
\hline $2 \mathrm{H}-1,44-46$ & 6.34 & 1.75 & 0.08 & 0.48 & 179 & 83.5 & 19.5 & 9.0 & 9.0 & 3.0 & 2.5 & 5.0 & 3.0 \\
\hline $2 \mathrm{H}-1,102-104$ & 6.90 & 4.08 & 2.38 & 2.01 & 191 & 51.0 & 10.0 & 5.5 & 6.0 & 0.0 & 47.0 & 12.5 & 2.3 \\
\hline $2 \mathrm{H}-1,134-136$ & 7.21 & 0.81 & 0.03 & 1.94 & 132 & 83.5 & 18.5 & 10.0 & 11.0 & 5.0 & 0.0 & 5.5 & 2.8 \\
\hline $2 \mathrm{H}-2,14-16$ & 7.50 & 0.42 & 0.10 & 0.22 & 109 & 74.0 & 17.0 & 7.0 & 7.0 & 2.5 & 0.0 & 1.0 & 3.3 \\
\hline $2 \mathrm{H}-2,44-46$ & 7.79 & 0.84 & 0.08 & 0.26 & 140 & 75.0 & 18.0 & 8.0 & 9.5 & 2.0 & 0.0 & 0.0 & 4.3 \\
\hline $2 \mathrm{H}-2,74-76$ & 8.08 & 0.40 & 0.00 & 0.11 & 129 & 75.5 & 15.5 & 6.0 & 5.0 & 3.0 & 0.0 & 2.0 & 2.8 \\
\hline $2 \mathrm{H}-2,102-104$ & 8.35 & 0.38 & 0.20 & 0.00 & 94 & 107.5 & 19.5 & 9.5 & 10.5 & 0.0 & 0.0 & 0.0 & 2.5 \\
\hline $2 \mathrm{H}-2,134-136$ & 8.66 & 1.47 & 0.20 & 1.57 & 168 & 97.0 & 21.5 & 13.0 & 9.5 & 2.0 & 0.0 & 12.5 & 2.5 \\
\hline $2 \mathrm{H}-3,14-16$ & 8.95 & 0.49 & 0.18 & 0.59 & 112 & 117.5 & 22.0 & 14.5 & 12.0 & 1.0 & 0.0 & 3.0 & 1.3 \\
\hline $2 \mathrm{H}-3,44-46$ & 9.24 & 0.28 & 0.13 & 0.06 & 102 & 117.0 & 26.0 & 14.5 & 14.0 & 2.5 & 0.0 & 0.0 & 2.0 \\
\hline $2 \mathrm{H}-3,74-76$ & 9.53 & 1.31 & 0.62 & 3.63 & 185 & 93.0 & 17.0 & 12.0 & 10.5 & 0.0 & 9.0 & 15.5 & 1.5 \\
\hline $2 \mathrm{H}-3,102-104$ & 9.80 & 0.66 & 0.19 & 1.59 & 137 & 109.5 & 21.5 & 10.5 & 12.0 & 0.0 & 0.0 & 6.5 & 2.3 \\
\hline $2 \mathrm{H}-3,134-136$ & 10.11 & 0.42 & 0.13 & 0.10 & 86 & 118.0 & 25.5 & 16.0 & 17.5 & 0.0 & 0.0 & 0.0 & 1.8 \\
\hline $2 \mathrm{H}-4,14-16$ & 10.40 & 0.73 & 0.11 & 0.21 & 136 & 107.0 & 19.5 & 11.5 & 12.0 & 2.5 & 2.0 & 0.0 & 2.3 \\
\hline $2 \mathrm{H}-4,44-46$ & 10.69 & 1.33 & 0.10 & 0.26 & 168 & 102.5 & 21.0 & 11.0 & 9.0 & 2.0 & 0.0 & 0.0 & 2.8 \\
\hline $2 \mathrm{H}-4,74-76$ & 10.98 & 0.76 & 0.15 & 0.55 & 156 & 99.5 & 17.5 & 8.0 & 9.0 & 1.0 & 0.0 & 1.5 & 2.3 \\
\hline $2 \mathrm{H}-4,102-104$ & 11.26 & 0.79 & 0.12 & 0.28 & 130 & 99.0 & 19.5 & 8.5 & 9.0 & 0.0 & 0.0 & 1.0 & 2.0 \\
\hline $2 \mathrm{H}-4,134-136$ & 11.57 & 1.43 & 0.14 & 1.18 & 152 & 93.5 & 23.5 & 9.5 & 10.0 & 1.0 & 0.0 & 6.5 & 2.8 \\
\hline $2 \mathrm{H}-5,44-46$ & 12.15 & 0.49 & 0.10 & 0.62 & 126 & 92.0 & 18.0 & 11.0 & 8.0 & 1.5 & 0.0 & 4.5 & 2.8 \\
\hline $2 \mathrm{H}-5,74-76$ & 12.44 & 0.95 & 0.00 & 0.17 & 162 & 87.5 & 21.0 & 6.0 & 9.5 & 1.0 & 0.0 & 0.0 & 4.0 \\
\hline $2 \mathrm{H}-5,102-104$ & 12.71 & 0.70 & 0.11 & 1.25 & 139 & 100.5 & 21.0 & 11.0 & 10.5 & 4.0 & 0.0 & 5.0 & 1.8 \\
\hline $2 \mathrm{H}-5,134-136$ & 13.02 & 0.79 & 0.00 & 0.05 & 124 & 96.5 & 21.5 & 10.5 & 10.5 & 2.5 & 0.0 & 0.0 & 2.3 \\
\hline $2 \mathrm{H}-6,14-16$ & 13.31 & 0.88 & 0.02 & 0.13 & 142 & 103.5 & 22.0 & 10.5 & 8.0 & 3.0 & 0.0 & 3.0 & 2.3 \\
\hline $2 \mathrm{H}-6,44-46$ & 13.60 & 3.70 & 0.10 & 1.12 & 201 & 77.0 & 18.5 & 11.5 & 10.0 & 0.0 & 0.0 & 13.5 & 3.8 \\
\hline $2 \mathrm{H}-6,74-76$ & 13.89 & 1.43 & 0.21 & 1.06 & 167 & 112.0 & 23.0 & 13.5 & 15.5 & 0.0 & 0.0 & 7.5 & 1.3 \\
\hline $2 \mathrm{H}-6,134-136$ & 14.47 & 1.10 & 0.15 & 1.65 & 133 & 80.5 & 21.5 & 8.0 & 7.0 & 2.0 & 2.0 & 5.5 & 1.5 \\
\hline $2 \mathrm{H}-7,14-16$ & 14.76 & 1.23 & 0.04 & 0.19 & 161 & 89.0 & 21.5 & 11.5 & 9.5 & 5.0 & 0.0 & 3.5 & 3.3 \\
\hline $2 \mathrm{H}-7,44-46$ & 15.05 & 1.22 & 0.04 & 0.43 & 160 & 76.0 & 19.0 & 8.0 & 9.5 & 0.0 & 0.0 & 2.5 & 3.0 \\
\hline $3 \mathrm{H}-1,14-16$ & 15.55 & 0.86 & 0.11 & 0.39 & 177 & 104.0 & 20.0 & 11.0 & 11.5 & 4.0 & 0.0 & 2.5 & 1.0 \\
\hline $3 \mathrm{H}-1,44-46$ & 15.84 & 0.75 & 0.14 & 0.08 & 146 & 114.0 & 21.0 & 10.0 & 8.5 & 3.0 & 0.0 & 0.0 & 2.5 \\
\hline $3 \mathrm{H}-1,74-76$ & 16.13 & 0.30 & 0.07 & 0.14 & 111 & 109.5 & 20.5 & 8.5 & 10.5 & 2.0 & 0.0 & 1.0 & 1.5 \\
\hline $3 \mathrm{H}-1,103-105$ & 16.41 & 0.90 & 0.07 & 0.08 & 133 & 82.0 & 15.0 & 9.5 & 10.5 & 3.0 & 0.0 & 0.0 & 3.3 \\
\hline $3 \mathrm{H}-1,135-137$ & 16.72 & 2.09 & 0.02 & 0.62 & 204 & 65.5 & 17.0 & 6.5 & 4.0 & 4.0 & 0.0 & 2.0 & 3.0 \\
\hline $3 \mathrm{H}-2,14-16$ & 17.00 & 0.45 & 0.12 & 0.46 & 125 & 100.0 & 17.5 & 11.0 & 10.0 & 2.0 & 0.0 & 0.0 & 2.8 \\
\hline $3 \mathrm{H}-2,44-46$ & 17.29 & 0.42 & 0.10 & 0.48 & 117 & 101.5 & 19.0 & 10.0 & 10.0 & 0.0 & 0.0 & 0.0 & 2.0 \\
\hline $3 \mathrm{H}-2,74-76$ & 17.58 & 0.97 & 0.04 & 0.55 & 154 & 95.0 & 20.0 & 8.5 & 9.0 & 2.0 & 0.0 & 4.0 & 2.8 \\
\hline $3 \mathrm{H}-2,103-105$ & 17.86 & 0.66 & 0.10 & 0.60 & 126 & 96.0 & 20.0 & 9.5 & 9.5 & 2.5 & 0.0 & 4.0 & 3.3 \\
\hline $3 \mathrm{H}-2,135-137$ & 18.17 & 1.20 & 0.05 & 0.40 & 162 & 83.5 & 18.0 & 8.0 & 9.0 & 1.5 & 0.0 & 0.0 & 3.3 \\
\hline $3 \mathrm{H}-3,14-16$ & 18.45 & 1.86 & 0.32 & 1.15 & 175 & 99.5 & 19.0 & 12.0 & 10.0 & 1.5 & 0.0 & 6.0 & 1.8 \\
\hline $3 \mathrm{H}-3,40-42$ & 18.71 & 0.42 & 0.08 & 0.85 & 114 & 90.5 & 15.5 & 9.0 & 8.0 & 4.0 & 0.0 & 2.5 & 3.5 \\
\hline $3 \mathrm{H}-3,74-76$ & 19.04 & 1.39 & 0.19 & 5.42 & 172 & 83.5 & 24.5 & 12.5 & 11.0 & 0.0 & 0.0 & 20.5 & 2.0 \\
\hline $3 \mathrm{H}-3,103-105$ & 19.32 & 0.98 & 0.19 & 0.87 & 139 & 106.5 & 21.5 & 12.5 & 12.0 & 0.0 & 0.0 & 2.0 & 3.0 \\
\hline $3 \mathrm{H}-3,135-137$ & 19.63 & 0.82 & 0.18 & 3.87 & 122 & 77.0 & 14.0 & 7.0 & 7.0 & 0.0 & 0.0 & 18.5 & 1.0 \\
\hline $3 \mathrm{H}-4,14-16$ & 19.91 & 0.35 & 0.26 & 0.24 & 110 & 114.0 & 23.5 & 13.5 & 13.5 & 0.0 & 0.0 & 0.0 & 2.3 \\
\hline $3 \mathrm{H}-4,44-46$ & 20.20 & 2.14 & 0.10 & 0.57 & 168 & 79.5 & 15.5 & 8.5 & 6.0 & 0.0 & 0.0 & 2.5 & 4.5 \\
\hline $3 \mathrm{H}-4,74-76$ & 20.49 & 0.40 & 0.23 & 0.20 & 101 & 87.0 & 21.0 & 10.0 & 7.0 & 4.0 & 0.0 & 0.0 & 2.0 \\
\hline $3 \mathrm{H}-4,103-105$ & 20.77 & 0.66 & 0.04 & 1.40 & 195 & 36.0 & 11.0 & 3.0 & 2.5 & 0.0 & 0.0 & 6.0 & 6.0 \\
\hline $3 \mathrm{H}-4,135-137$ & 21.08 & 0.39 & 0.09 & 0.60 & 89 & 92.0 & 18.0 & 9.5 & 9.0 & 0.0 & 0.0 & 2.0 & 2.8 \\
\hline $3 \mathrm{H}-5,14-16$ & 21.36 & 0.48 & 0.01 & 1.01 & 128 & 82.5 & 15.0 & 8.5 & 7.0 & 1.5 & 0.0 & 2.5 & 3.0 \\
\hline $3 \mathrm{H}-5,44-46$ & 21.65 & 0.41 & 0.01 & 0.09 & 134 & 84.5 & 17.0 & 6.0 & 5.0 & 3.0 & 0.0 & 0.0 & 4.0 \\
\hline $3 \mathrm{H}-5,73-75$ & 21.93 & 0.38 & 0.00 & 0.02 & 115 & 79.5 & 19.5 & 10.5 & 8.0 & 3.5 & 0.0 & 2.0 & 2.8 \\
\hline $3 \mathrm{H}-5,103-105$ & 22.22 & 0.46 & 0.00 & 1.11 & 123 & 73.0 & 17.0 & 8.5 & 7.0 & 3.5 & 0.0 & 2.0 & 2.8 \\
\hline $3 \mathrm{H}-6,14-16$ & 22.82 & 1.11 & 0.12 & 2.06 & 164 & 101.5 & 20.0 & 10.5 & 10.5 & 1.5 & 0.0 & 9.0 & 1.3 \\
\hline $3 \mathrm{H}-6,44-46$ & 23.11 & 0.92 & 0.21 & 1.94 & 166 & 114.0 & 19.5 & 12.0 & 11.0 & 1.5 & 0.0 & 10.5 & 1.8 \\
\hline $3 \mathrm{H}-6,73-75$ & 23.39 & 1.38 & 0.12 & 0.84 & 183 & 77.0 & 19.0 & 9.0 & 8.5 & 0.0 & 0.0 & 6.0 & 3.0 \\
\hline $3 \mathrm{H}-6,103-105$ & 23.68 & 1.05 & 1.41 & 1.87 & 140 & 87.0 & 18.0 & 13.0 & 13.0 & 2.0 & 32.5 & 9.0 & 1.3 \\
\hline $3 \mathrm{H}-6,135-137$ & 23.99 & 0.53 & 3.11 & 0.55 & 93 & 79.0 & 13.0 & 10.0 & 8.0 & 0.0 & 63.0 & 3.5 & 1.3 \\
\hline
\end{tabular}


Table 2 (continued).

\begin{tabular}{|c|c|c|c|c|c|c|c|c|c|c|c|c|c|}
\hline $\begin{array}{l}\text { Core, section, } \\
\text { interval }(\mathrm{cm})\end{array}$ & $\begin{array}{l}\text { Depth } \\
\text { (mbsf) }\end{array}$ & $\begin{array}{c}\mathrm{C}_{\mathrm{org}} \\
(\mathrm{wt} \%)\end{array}$ & $\begin{array}{l}\mathrm{C}_{\text {carb }} \\
(\mathrm{wt} \%)\end{array}$ & $\begin{array}{c}\mathrm{S}_{\mathrm{tot}} \\
(\mathrm{wt} \%)\end{array}$ & $\begin{array}{l}\text { Gray } \\
\text { value }\end{array}$ & $\begin{array}{c}\text { Quartz } \\
(\mathrm{cps} / 10)\end{array}$ & $\begin{array}{l}\text { Plagioclase } \\
(\mathrm{cps} / 10)\end{array}$ & $\begin{array}{c}\text { Illite } \\
(\mathrm{cps} / 10)\end{array}$ & $\begin{array}{c}\text { Kaolinite + chlorite } \\
(\mathrm{cps} / 10)\end{array}$ & $\begin{array}{l}\text { Smectite } \\
(\mathrm{cps} / 10)\end{array}$ & $\begin{array}{l}\text { Calcite } \\
(\mathrm{cps} / 10)\end{array}$ & $\begin{array}{l}\text { Pyrite } \\
(\mathrm{cps} / 10)\end{array}$ & $\begin{array}{r}\text { Opal-A } \\
(\mathrm{cps} / 10)\end{array}$ \\
\hline $3 \mathrm{H}-7,14-16$ & 24.27 & 0.92 & 2.97 & 0.69 & 140 & 67.5 & 15.5 & 8.5 & 8.0 & 3.0 & 62.0 & 4.0 & 1.8 \\
\hline $4 \mathrm{H}-1,14-17$ & 25.05 & 0.37 & 0.12 & 0.61 & 135 & 108.5 & 20.0 & 9.5 & 9.5 & 0.0 & 0.0 & 1.5 & 2.8 \\
\hline $4 \mathrm{H}-1,44-46$ & 25.34 & 0.43 & 0.15 & 0.85 & 124 & 104.0 & 26.5 & 16.0 & 14.5 & 4.0 & 0.0 & 3.0 & 2.8 \\
\hline $4 \mathrm{H}-1,74-76$ & 25.64 & 0.94 & 0.23 & 0.62 & 190 & 67.5 & 18.5 & 8.5 & 7.0 & 3.5 & 0.0 & 1.5 & 2.5 \\
\hline $4 \mathrm{H}-1,102-104$ & 25.91 & 0.49 & 0.56 & 0.23 & 133 & 73.0 & 18.0 & 10.0 & 8.0 & 3.0 & 0.0 & 0.0 & 2.3 \\
\hline $4 \mathrm{H}-1,135-137$ & 26.23 & 0.67 & 0.03 & 0.73 & 150 & 75.0 & 18.0 & 6.0 & 6.5 & 3.0 & 0.0 & 4.5 & 3.5 \\
\hline $4 \mathrm{H}-2,20-22$ & 26.58 & 1.52 & 0.51 & 0.72 & 171 & 67.0 & 18.0 & 4.5 & 5.5 & 3.0 & 8.0 & 6.0 & 4.0 \\
\hline $4 \mathrm{H}-2,44-46$ & 26.81 & 1.54 & 0.06 & 1.47 & 187 & 65.0 & 15.5 & 5.5 & 5.5 & 4.5 & 0.0 & 7.0 & 2.3 \\
\hline $4 \mathrm{H}-2,74-76$ & 27.11 & 2.32 & 0.09 & 1.56 & 204 & 74.0 & 15.0 & 7.0 & 8.5 & 2.5 & 0.0 & 6.0 & 3.3 \\
\hline $4 \mathrm{H}-2,103-105$ & 27.39 & 1.09 & 0.07 & 0.64 & 218 & 30.5 & 7.0 & 2.5 & 3.0 & 2.0 & 0.0 & 1.0 & 5.8 \\
\hline $4 \mathrm{H}-2,135-137$ & 27.71 & 1.33 & 0.09 & 1.40 & 182 & 70.0 & 14.5 & 8.5 & 7.5 & 4.5 & 0.0 & 5.5 & 4.0 \\
\hline $4 \mathrm{H}-3,14-16$ & 27.99 & 0.73 & 0.13 & 0.69 & 129 & 99.0 & 19.5 & 10.5 & 12.5 & 2.5 & 0.0 & 2.5 & 2.5 \\
\hline $4 \mathrm{H}-3,44-46$ & 28.29 & 0.57 & 0.23 & 0.49 & & 15.5 & 6.0 & 0.0 & 2.0 & 0.0 & 4.5 & 2.0 & 4.8 \\
\hline $4 \mathrm{H}-3,74-76$ & 28.58 & 0.81 & 0.16 & 0.28 & 170 & 95.5 & 18.0 & 10.0 & 9.5 & 3.5 & 0.0 & 4.5 & 3.3 \\
\hline $4 \mathrm{H}-3,102-104$ & 28.85 & 0.41 & 2.11 & 1.16 & 102 & 73.5 & 16.5 & 8.5 & 9.5 & 1.5 & 48.0 & 3.0 & 1.3 \\
\hline $4 \mathrm{H}-3,135-137$ & 29.18 & 3.12 & 1.53 & 2.32 & 155 & 63.0 & 12.0 & 6.5 & 7.5 & 4.0 & 33.0 & 10.5 & 3.5 \\
\hline $4 \mathrm{H}-4,13-15$ & 29.45 & 0.53 & 0.62 & 0.64 & 107 & 77.5 & 17.0 & 8.0 & 7.0 & 4.0 & 13.0 & 2.5 & 2.0 \\
\hline $4 \mathrm{H}-4,44-46$ & 29.76 & 0.72 & 0.07 & 0.84 & 145 & 79.0 & 18.5 & 11.5 & 10.0 & 2.5 & 0.0 & 0.0 & 2.8 \\
\hline $4 \mathrm{H}-4,74-76$ & 30.05 & 0.33 & 0.12 & 0.52 & 115 & 93.0 & 18.0 & 6.5 & 8.0 & 4.5 & 0.0 & 2.0 & 2.5 \\
\hline $4 \mathrm{H}-4,102-104$ & 30.33 & 3.10 & 2.40 & 2.91 & 195 & 61.0 & 12.5 & 6.5 & 7.0 & 2.0 & 49.0 & 11.0 & 2.0 \\
\hline $4 \mathrm{H}-4,135-137$ & 30.65 & 2.52 & 6.29 & 1.16 & 177 & 24.5 & 4.0 & 2.5 & 2.5 & 2.0 & 133.5 & 6.0 & 2.3 \\
\hline $4 \mathrm{H}-5,14-16$ & 30.94 & 0.91 & 0.10 & 1.01 & 157 & 75.5 & 17.5 & 7.5 & 7.5 & 2.0 & 0.0 & 5.0 & 3.0 \\
\hline $4 \mathrm{H}-5,43-45$ & 31.22 & 0.30 & 2.77 & 0.16 & 90 & 63.5 & 14.5 & 8.5 & 9.5 & 3.5 & 63.0 & 0.0 & 2.5 \\
\hline $4 \mathrm{H}-5,71-73$ & 31.49 & 1.37 & 0.10 & 1.53 & 168 & 64.5 & 19.0 & 8.0 & 7.0 & 2.5 & 0.0 & 4.5 & 4.3 \\
\hline $4 \mathrm{H}-5,102-104$ & 31.80 & 0.71 & 0.04 & 0.52 & 143 & 90.0 & 17.0 & 8.0 & 7.0 & 3.5 & 0.0 & 0.0 & 2.0 \\
\hline $4 \mathrm{H}-5,135-137$ & 32.12 & 0.80 & 0.02 & 0.51 & 139 & 74.5 & 18.5 & 4.5 & 5.5 & 4.5 & 0.0 & 3.5 & 3.0 \\
\hline $4 \mathrm{H}-6,14-16$ & 32.41 & 0.57 & 0.06 & 0.53 & 133 & 73.0 & 16.5 & 7.0 & 7.0 & 4.0 & 2.0 & 3.5 & 1.5 \\
\hline $4 \mathrm{H}-6,44-46$ & 32.70 & 1.01 & 0.07 & 2.46 & 180 & 91.5 & 21.5 & 12.5 & 8.5 & 0.0 & 0.0 & 10.5 & 3.3 \\
\hline $4 \mathrm{H}-6,73-75$ & 32.99 & 1.24 & 0.13 & 2.07 & 180 & 104.5 & 19.5 & 13.5 & 12.5 & 5.5 & 0.0 & 8.5 & 3.0 \\
\hline $4 \mathrm{H}-6,102-104$ & 33.27 & 1.15 & 0.14 & 2.87 & 179 & 95.5 & 22.0 & 12.0 & 8.5 & 0.0 & 0.0 & 13.0 & 2.8 \\
\hline $4 \mathrm{H}-6,135-137$ & 33.59 & 1.15 & 1.87 & 1.86 & 173 & 75.0 & 15.0 & 8.5 & 7.5 & 1.5 & 36.5 & 10.0 & 1.8 \\
\hline $5 \mathrm{H}-1,14-16$ & 34.54 & 0.29 & 0.00 & 0.56 & 126 & 66.5 & 18.5 & 4.5 & 4.5 & 1.5 & 0.0 & 1.5 & 4.3 \\
\hline $5 \mathrm{H}-1,44-46$ & 34.83 & 3.25 & 0.05 & 0.63 & 199 & 66.0 & 15.0 & 9.0 & 3.5 & 2.5 & 0.0 & 0.0 & 4.0 \\
\hline $5 \mathrm{H}-1,74-76$ & 35.12 & 0.60 & 0.10 & 0.28 & 134 & 88.0 & 16.5 & 9.0 & 9.0 & 4.5 & 0.0 & 0.0 & 3.0 \\
\hline $5 \mathrm{H}-1,103-105$ & 35.41 & 0.99 & 0.23 & 1.63 & 171 & 104.0 & 21.0 & 9.5 & 10.0 & 0.0 & 0.0 & 14.0 & 2.0 \\
\hline $5 \mathrm{H}-1,132-134$ & 35.69 & 1.30 & 0.56 & 1.41 & 145 & 88.0 & 18.0 & 11.5 & 10.5 & 2.0 & 11.5 & 5.5 & 2.8 \\
\hline $5 \mathrm{H}-2,17-19$ & 36.02 & 0.42 & 0.08 & 0.84 & 107 & 89.0 & 17.5 & 11.0 & 10.0 & 4.0 & 0.0 & 3.0 & 3.3 \\
\hline $5 \mathrm{H}-2,44-46$ & 36.28 & 0.61 & 0.20 & 0.83 & 148 & 98.0 & 18.5 & 12.0 & 10.5 & 0.0 & 2.5 & 4.0 & 2.0 \\
\hline $5 \mathrm{H}-2,74-76$ & 36.57 & 0.85 & 0.10 & 0.54 & 148 & 103.0 & 19.0 & 11.0 & 9.0 & 3.5 & 0.0 & 0.0 & 2.5 \\
\hline $5 \mathrm{H}-2,103-105$ & 36.86 & 0.51 & 0.53 & 0.81 & 170 & 103.0 & 20.5 & 10.0 & 11.0 & 2.0 & 9.5 & 4.0 & 3.3 \\
\hline $5 \mathrm{H}-2,132-134$ & 37.14 & 3.87 & 0.13 & 2.91 & 201 & 73.0 & 17.0 & 9.0 & 9.0 & 2.0 & 0.0 & 12.0 & 3.3 \\
\hline $5 \mathrm{H}-3,14-16$ & 37.44 & 0.41 & 0.13 & 0.71 & 113 & 92.0 & 17.5 & 8.0 & 10.0 & 2.0 & 0.0 & 4.0 & 3.5 \\
\hline $5 \mathrm{H}-3,44-46$ & 37.73 & 1.99 & 0.06 & 1.11 & 161 & 73.0 & 16.0 & 8.5 & 7.0 & 2.0 & 0.0 & 2.0 & 3.3 \\
\hline $5 \mathrm{H}-3,74-76$ & 38.02 & 1.07 & 0.07 & 0.29 & 143 & 85.0 & 17.0 & 8.5 & 4.0 & 2.0 & 0.0 & 1.0 & 2.8 \\
\hline $5 \mathrm{H}-3,102-104$ & 38.30 & 0.42 & 0.00 & 0.16 & 108 & 81.0 & 21.0 & 9.5 & 8.5 & 6.0 & 0.0 & 1.0 & 2.5 \\
\hline $5 \mathrm{H}-3,132-134$ & 38.59 & 0.42 & 0.01 & 2.48 & 117 & 61.0 & 13.0 & 6.5 & 5.5 & 2.0 & 0.0 & 7.0 & 3.3 \\
\hline $5 \mathrm{H}-4,14-16$ & 38.89 & 0.86 & 0.27 & 0.90 & 153 & 110.5 & 23.0 & 12.0 & 12.5 & 0.0 & 0.0 & 4.5 & 1.8 \\
\hline $5 \mathrm{H}-4,43-45$ & 39.17 & 0.81 & 0.44 & 0.78 & 128 & 97.5 & 18.5 & 10.0 & 9.5 & 0.0 & 5.5 & 3.5 & 2.3 \\
\hline $5 \mathrm{H}-4,74-76$ & 39.47 & 0.53 & 0.65 & 0.71 & 120 & 87.0 & 16.0 & 9.5 & 9.5 & 2.0 & 11.0 & 3.0 & 2.5 \\
\hline $5 \mathrm{H}-4,103-105$ & 39.75 & 1.88 & 0.07 & 0.87 & 171 & 72.0 & 14.5 & 9.0 & 6.0 & 2.5 & 0.0 & 3.5 & 3.5 \\
\hline $5 \mathrm{H}-4,131-133$ & 40.03 & 1.35 & 0.21 & 0.79 & 129 & 76.0 & 15.0 & 11.0 & 11.0 & 1.5 & 0.0 & 3.0 & 2.0 \\
\hline $5 \mathrm{H}-5,11-13$ & 40.32 & 1.46 & 1.58 & 0.86 & 169 & 68.0 & 10.5 & 10.0 & 8.5 & 1.0 & 34.5 & 5.0 & 2.5 \\
\hline $5 \mathrm{H}-5,43-45$ & 40.62 & 0.39 & 0.80 & 0.06 & 95 & 78.5 & 14.0 & 7.0 & 4.5 & 2.0 & 19.0 & 1.0 & 2.8 \\
\hline $5 \mathrm{H}-5,74-76$ & 40.92 & 0.36 & 0.29 & 0.46 & 105 & 75.0 & 17.5 & 8.0 & 6.5 & 2.0 & 2.0 & 1.5 & 3.5 \\
\hline $5 \mathrm{H}-5,103-105$ & 41.20 & 0.36 & 0.14 & 0.70 & 102 & 77.5 & 15.5 & 11.0 & 11.0 & 2.5 & 0.0 & 0.0 & 2,3 \\
\hline $5 \mathrm{H}-5,131-133$ & 41.48 & 0.34 & 3.58 & 0.04 & 50 & 49.0 & 9.0 & 7.0 & 6.0 & 0.0 & 79.0 & 0.0 & 1.3 \\
\hline $5 H-6,14-16$ & 41.79 & 0.29 & 1.21 & 0.25 & 96 & 46.0 & 11.5 & 4.0 & 4.5 & 0.0 & 32.0 & 0.0 & 3.8 \\
\hline $5 \mathrm{H}-6,44-46$ & 42.08 & 1.19 & 0.11 & 1.13 & 177 & 78.0 & 16.0 & 11.5 & 9.5 & 1.5 & 0.0 & 6.5 & 4.8 \\
\hline $5 \mathrm{H}-6,81-83$ & 42.44 & 1.08 & 0.16 & 2.64 & 200 & 103.5 & 18.5 & 6.5 & 9.5 & 1.5 & 0.0 & 11.0 & 2.3 \\
\hline $5 \mathrm{H}-6,102-104$ & 42.64 & 1.96 & 1.42 & 3.07 & 210 & 76.0 & 13.0 & 6.5 & 6.0 & 0.0 & 27.0 & 11.5 & 2.0 \\
\hline $5 \mathrm{H}-6,131-133$ & 42.93 & 0.37 & 0.23 & 1.01 & 133 & 115.5 & 34.0 & 13.5 & 11.0 & 0.0 & 0.0 & 2.5 & 2.3 \\
\hline $5 \mathrm{H}-7,14-16$ & 43.24 & 1.46 & 0.83 & 1.16 & 176 & 79.5 & 17.0 & 6.5 & 7.0 & 0.0 & 13.5 & 4.0 & 3.3 \\
\hline $5 \mathrm{H}-7,43-45$ & 43.52 & 1.43 & 1.18 & 1.69 & 170 & 87.0 & 17.0 & 10.5 & 10.0 & 0.0 & 12.5 & 6.0 & 2.3 \\
\hline $6 \mathrm{H}-1,13-15$ & 44.03 & 0.72 & 0.19 & 1.18 & 129 & 88.0 & 17.0 & 8.0 & 6.0 & 0.0 & 0.0 & 3.0 & 2.5 \\
\hline $6 \mathrm{H}-1,43-45$ & 44.32 & 2.52 & 0.09 & 1.23 & 111 & 71.0 & 13.0 & 8.0 & 7.0 & 0.0 & 0.0 & 4.0 & 3.8 \\
\hline $6 \mathrm{H}-1,74-76$ & 44.62 & 0.35 & 0.00 & 0.30 & 115 & 80.0 & 17.0 & 10.0 & 6.5 & 2.5 & 0.0 & 0.0 & 3.3 \\
\hline $6 \mathrm{H}-1,102-104$ & 44.89 & 0.27 & 0.00 & 0.07 & 93 & 86.0 & 13.0 & 6.5 & 7.0 & 2.5 & 0.0 & 1.5 & 2.5 \\
\hline $6 \mathrm{H}-1,132-134$ & 45.18 & 2.67 & 0.73 & 2.13 & 168 & 73.0 & 14.0 & 5.0 & 6.5 & 0.0 & 14.0 & 9.0 & 3.3 \\
\hline $6 \mathrm{H}-2,13-15$ & 45.48 & 0.37 & 0.11 & 0.64 & 109 & 112.0 & 19.5 & 9.0 & 7.5 & 1.5 & 0.0 & 3.0 & 1.8 \\
\hline $6 \mathrm{H}-2,43-45$ & 45.76 & 0.40 & 0.16 & 0.83 & 149 & 69.0 & 15.0 & 9.5 & 9.0 & 1.5 & 0.0 & 3.0 & 3.8 \\
\hline
\end{tabular}




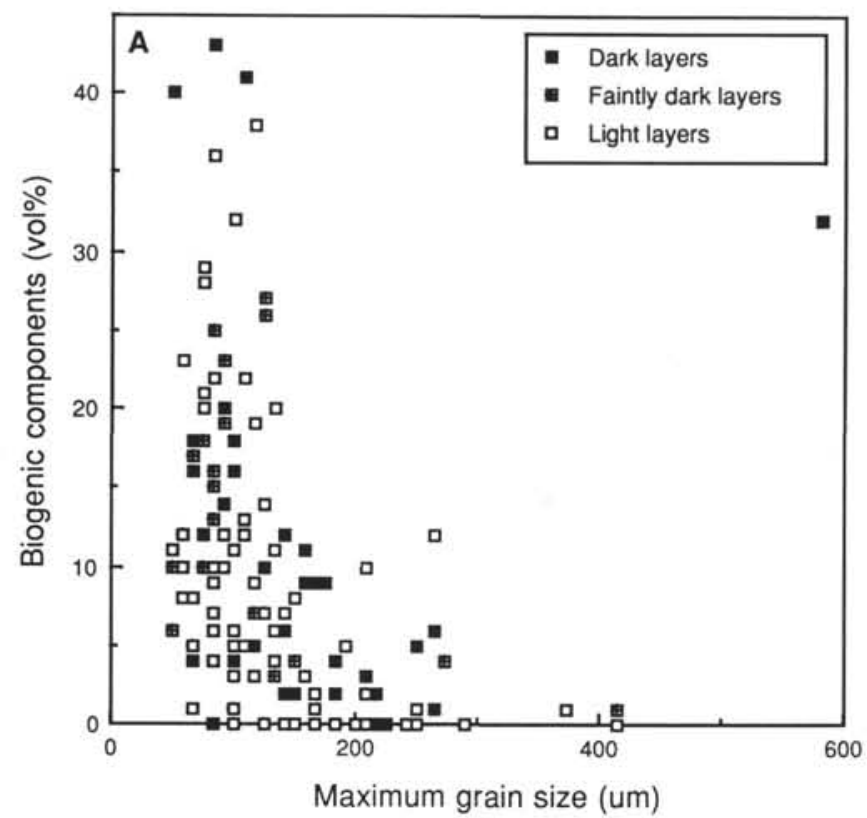

Figure 5. The relationship between the maximum grain size of detrital silts and the biogenic component (diatoms + radiolarians + silicoflagellates + sponges + foraminifers) for samples from the upper Quaternary sediments at Site 797. The maximum grain size and the biogenic component was based on smear slide analysis.

sediments. However, smectite does not show any correlation with other detrital minerals such as quartz (Fig. 6D).

\section{Carbon and Sulfur Contents}

The $\mathrm{C}_{\mathrm{org}}$ content of the sediments is variable, ranging from 0.27 to $4.32 \mathrm{wt} \%$ (Table 2). The $\mathrm{C}_{\mathrm{org}}$ content tends to be higher in dark layers, suggesting that the presence of organic carbon contributes to the dark color (Potter et al., 1980; Tada, 1991). This tendency is clearly illustrated when the gray value of the sediments is plotted against their $\mathrm{C}_{\text {org }}$ content (Fig. 7). The two have a clear positive correlation, but the regression is not linear but logarithmic and the coefficient is not very high $\left(r^{2}=0.63\right)$. The metastable FeS may also contribute to the black color (Berner, 1971), which would partly explain the scatter in the correlation.

The $\mathrm{C}_{\text {carb }}$ content of the sediments is variable, ranging from 0.00 to $6.29 \mathrm{wt} \%$ (Table 1). The $\mathrm{C}_{\text {carb }}$ is present as calcite. There is a strong positive correlation between the calcite peak height and $\mathrm{C}_{\text {carb }}$ content $\left(r^{2}=0.98\right)$, suggesting that calcite is the only carbonate mineral present and its crystallinity is uniform.

The $S_{\text {tot }}$ content of the samples is also variable, ranging from 0.00 to $5.42 \mathrm{wt} \%$. The $\mathrm{S}_{\text {tot }}$ content tends to be higher in the dark layers, but the association is not as clear as that for the $\mathrm{C}_{\text {org }}$ content. The weak positive correlation between gray value and $S_{\text {tot }}\left(r^{2}=0.22\right)$ may indicate the contribution of FeS to the darkness. It is also possible that the positive correlation is simply an artifact of the correlation between gray value and $\mathrm{C}_{\mathrm{org}}\left(\mathrm{r}^{2}=0.63\right)$ and that between $\mathrm{C}_{\mathrm{org}}$ and $\mathrm{S}_{\mathrm{tot}}\left(\mathrm{r}^{2}=0.23\right)$.

Sulfur in marine sediments is present dominantly as pyrite (Berner and Raiswell, 1983). The strong positive correlation between the pyrite peak height and $\mathrm{S}_{\text {tot }}$ for the upper Quaternary samples from Site $797\left(r^{2}=0.83\right)$ also supports the inference that sulfur is present dominantly as pyrite, although the presence of $\mathrm{FeS}$ in small amounts is possible especially in the upper part of the sequence studied.

\section{Vertical Variation in Sediment Composition and "Tuning" of the Sediment Age to the Oxygen Isotopic Stages at Site 797}

Vertical variations in XRD peak intensities of quartz, illite, and smectite, contents of $\mathrm{C}_{\text {carb }}, \mathrm{C}_{\text {org }}, \mathrm{S}_{\text {tot }}$, and diatoms, the diatom number, the maximum grain size, and the gray value are shown in Figure 8A-J. In order to examine the interrelation between sediment composition and glacial-interglacial conditions, the stacked $\delta^{18} \mathrm{O}$ curve presented by Imbrie et al. (1984) is also shown. Oscillation in the $\delta^{18} \mathrm{O}$ is interpreted as being strongly influenced by changes in the volume of ice (Imbrie et al., 198), which in turn reflects in the glacio-eustatic sea-level changes.

\section{"Tuning" to the Oxygen Isotope Stages}

The $\delta^{18} \mathrm{O}$ curve presented in Figure $8 \mathrm{~K}$ is interpolated between five chronologically controlled levels; namely, the sediment-water interface at 0 mbsf ( $0 \mathrm{ka})$, the Aso- 4 ash layer at $5.8 \mathrm{mbsf}(88 \mathrm{ka})$, the $\mathrm{LO}$ of R. curvirostris at $16.6 \mathrm{mbsf}(0.30 \mathrm{Ma})$, the LO of P. lacunosa at $23.8 \mathrm{mbsf}(0.46 \mathrm{Ma})$, and the Brunhes/Matuyama boundary at 38.3 mbsf (0.73 Ma).

A comparison between the shape of the $\delta^{18} \mathrm{O}$ curve with that of the variation in the diatom content and the diatom number indicates that the three curves show similar trends. Positive and negative peaks of the diatom content and/or the diatom number corresponding to positive and negative peaks of the $\delta^{18} \mathrm{O}$ curve. As will be discussed later, the abundance of diatoms is probably controlled by glacio-eustatic sea-level changes, as such it seems plausible to use the diatom content (or the diatom number) curve as the substitute for the $\delta^{18}$ Ocurve. This substitution is necessary because it is not possible to take a continuous $\delta^{18} \mathrm{O}$ record from the upper Quaternary sediments at the basinal sites of the Japan Sea in which the occurrence of calcareous fossils is only sporadic. Through cross-peak correlation of the three curves, we have "tuned" the ages of the sediments at Site 797 to the oxygen isotopic stages presented by Imbrie et al. (1984).

The stratigraphic levels at which dropstones occur at Site 795 are projected to Site 797 (Fig. 8J) based on the correlation of the dark and light layers. Four dropstone levels fall in glacial stages $6,12,16$, and 20 . These glacial stages are characterized by rather strong negative peaks of the $\delta^{18} \mathrm{O}$ curve. This fact gives further support to our correlation of the Site 797 sediments with the oxygen isotopic stages.

\section{Vertical Variation in Sediment Composition}

The content of quartz and illite tends to be higher during glacial stages and diminished during interglacial stages (Fig. 8A, B). Exceptions are found within glacial stages 4 and 8 when illite content is not increased and interglacial stages 3 and 13 when illite content is not diminished. The content of plagioclase and kaolinite plus chlorite shows similar trends. Lower content of these detrital minerals during interglacial stages is partly due to the dilution effect by biogenic silica. However, the variation in the content by a factor of 2 for quartz and plagioclase and by a factor of 3 to for illite and kaolinite plus chlorite, when highly calcareous samples are excluded, is too large to be explained solely by the dilution effect of biogenic silica (opal-A) whose amounts are less than $30 \pm 10 \mathrm{wt} \%$.

The content of smectite, on the other hand, seems to show the opposite trend (Fig. 8C); that is, its content tends to be increased during interglacial stages. Exceptions are found during glacial stages 8 and 16 when its content remains high. However, we need further investigation to confirm this trend, since the method we adopted in this study is not adequate to quantify smectite contents. 

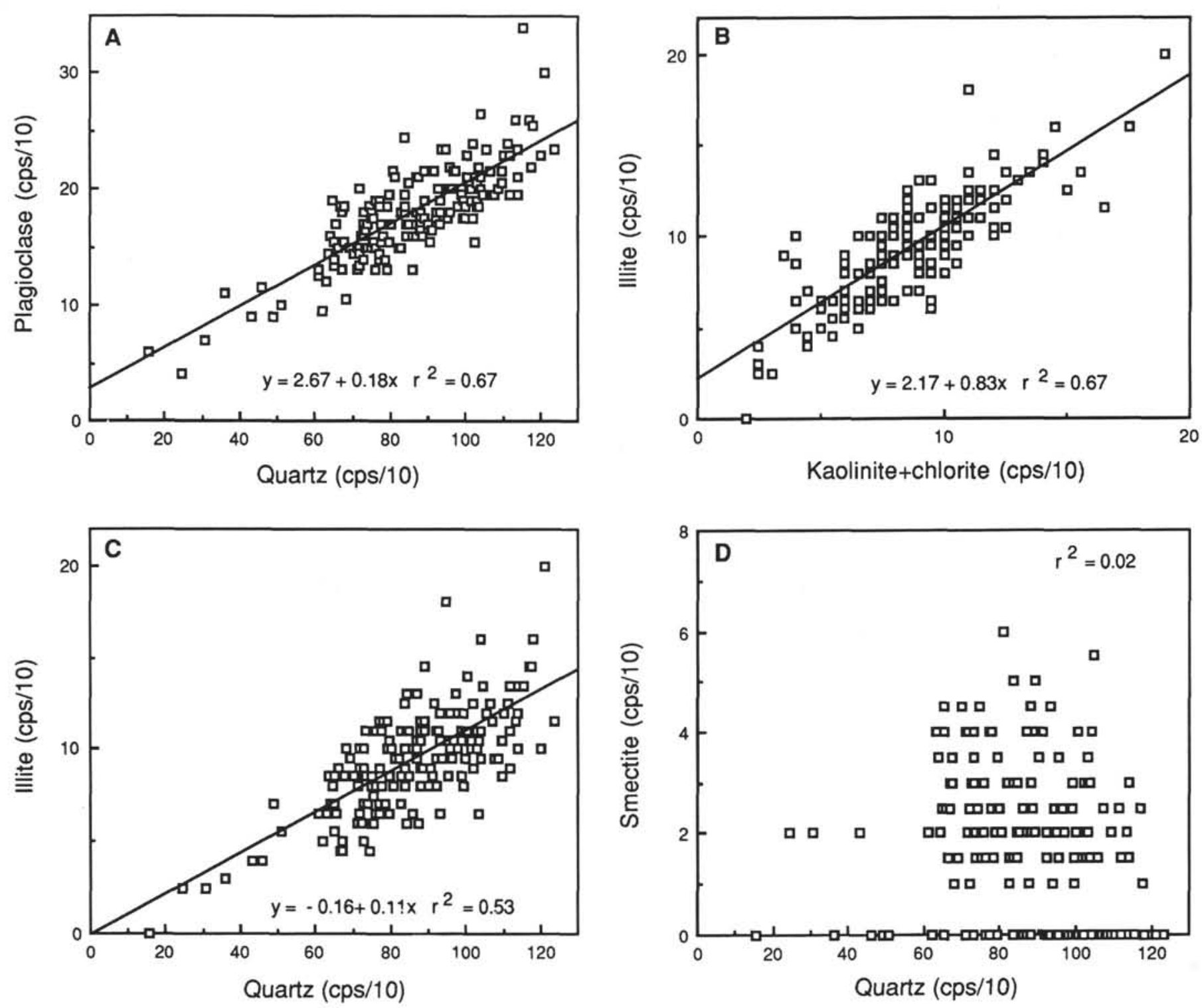

Figure 6. The relationships between quartz and plagioclase peak heights (A), kaolinite plus chlorite and illite peak heights (B), quartz and illite peak heights (C), and quartz and smectite peak heights (D) for samples from the upper Quaternary sediments at Site 797.

The maximum grain size tends to be larger during glacial stages (Fig. $8 \mathrm{H}$ ). The maximum grain sizes larger than $150 \mu \mathrm{m}$ are generally found during glacial stages. An exception is found during interglacial stages 7 and 11. The two levels within stages 7 and 11, at which the maximum grain sizes are larger than $150 \mu \mathrm{m}$, probably correspond to cooler events during interglacial stages which are represented by negative peaks of the $\delta^{18} \mathrm{O}$ curve. The maximum grain size are generally about $80 \mu \mathrm{m}$ during the interglacial stages and $150-250 \mu \mathrm{m}$ during glacial stages. However, two samples from stages 8 and 10 show a maximum grain size exceeding $400 \mu \mathrm{m}$.

$\mathrm{C}_{\text {org }}$ contents higher than $1.5 \mathrm{wt} \%$ occur only during interglacial stages with one exception (Fig. 8E). The exception is Sample 127-797B-3H, $135-137 \mathrm{~cm}$, at $16.72 \mathrm{mbsf}$ which may correspond to isotopic event 8.6 of Imbrie et al. (1984). This event is characterized by a slightly heavy $\delta^{18} \mathrm{O}$ value indicating a slightly lowered sea level. During interglacial stages, sediments containing $>1.5 \mathrm{wt} \% \mathrm{C}_{\mathrm{org}}$ tend to be found at proximal to stage boundaries and cooler events which are characterized by inter- mediate to slightly light $\delta^{18} \mathrm{O}$ values corresponding to intermediate to slightly raised sea levels.

The gray value profile contains a high frequency ( $>2$ cycles $/ \mathrm{m}$ ) fluctuation within several stratigraphic intervals. Such intervals tend to coincide with the intervals in which high $\mathrm{C}_{\text {org }}$ values occur (Fig. 8E, F). Further comparison of the variation in the $\mathrm{C}_{\mathrm{org}}$ content with the gray value shows that samples with high $\mathrm{C}_{\mathrm{org}}$ contents $(>1.5 \mathrm{wt} \%)$ coincide with narrow positive peaks of the gray value profile with values larger than 180. This observation suggests that sediments with high $\mathrm{C}_{\text {org }}$ contents occur within thin dark layers. This point is illustrated in Figure 9. The figure shows that $\mathrm{C}_{\mathrm{org}}$ content in dark layers thinner than $50 \mathrm{~cm}$ ranges from 0.5 to $4.4 \mathrm{wt} \%$, whereas those of dark layers thicker than $50 \mathrm{~cm}$ range from 0.9 to $1.4 \mathrm{wt} \%$. An exception is a sample from $68 \mathrm{~cm}$ thick dark layer I which contains $2.32 \mathrm{wt} \% \mathrm{C}_{\text {org }}$.

The $\mathrm{S}_{\mathrm{tot}}$ content, on the other hand, tends to be higher during glacial stages (Fig. 8G). The $\mathrm{S}_{\text {tot }}$ content is generally below $1 \mathrm{wt} \%$ and rarely exceeds 2 wt $\%$ during interglacial stages, whereas contents exceeding 


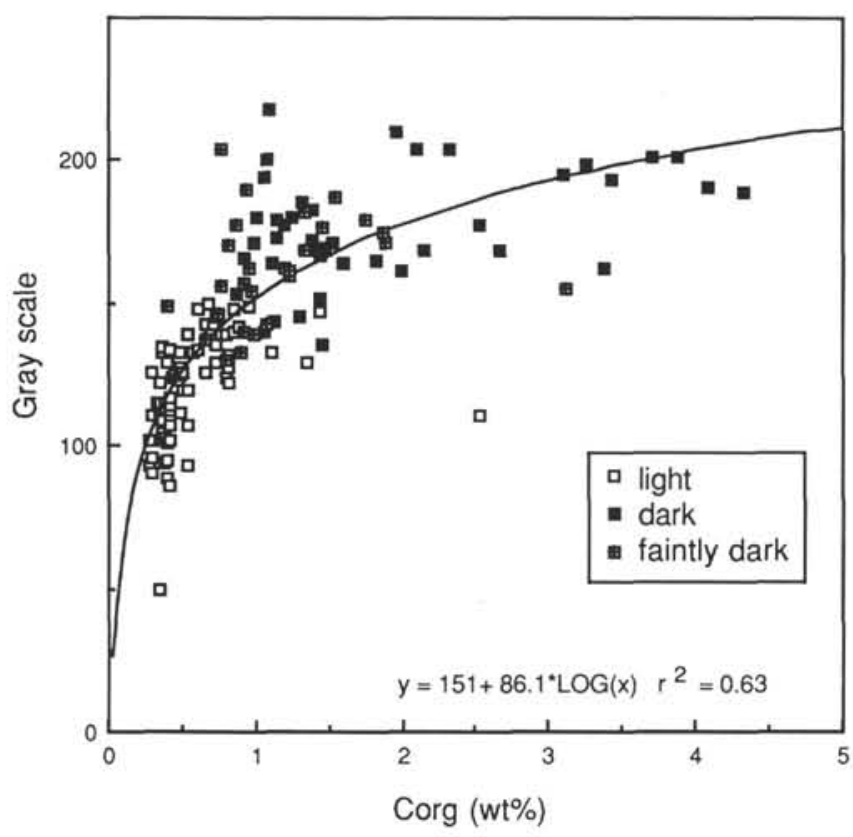

Figure 7. The relationship between the $\mathrm{C}_{\text {org }}$ content and gray value of the samples from the upper Quaternary sediments at Site 797.

$2 \mathrm{wt} \%$ are common during glacial stages. Exception is found during stage 8 , during which $\mathrm{S}_{\text {tot }}$ contents are below $1 \mathrm{wt} \%$. A comparison between the vertical variation in $\mathrm{S}_{\text {tot }}$ and the gray value profile shows that thick dark layers such as $\mathrm{A}, \mathrm{C}, \mathrm{H}$, and $\mathrm{K}$, which occur within glacial stages, are characterized by high $\mathrm{S}_{\text {tot }}$ contents.

The occurrence of samples with $\mathrm{C}_{\text {carb }}$ contents larger than $1 \mathrm{wt} \%$ is limited. Such samples tend to be found during interglacial stages (Fig 8D). Exceptions are Samples 127-797B-3H-6, 103-105 cm, and 127-797B-3H-6, 135-137 cm, which occur in glacial stage 12 . A comparison of the variation of $\mathrm{C}_{\text {carb }}$ with $\mathrm{C}_{\mathrm{org}}$ indicates that high $\mathrm{C}_{\text {cart }}$ samples are closely associated but not necessarily coincident with high $\mathrm{C}_{\text {org }}$ samples.

In summary, sediments deposited during glacial stages are generally characterized by larger maximum grain size of detrital silts, higher contents of detrital minerals (except smectite), and lower contents of biogenic silica (mainly diatoms) than are sediments deposited during interglacial stages. Dark layers deposited during glacial stages tend to be thicker, contain higher amount of $S_{\text {tot }}$ and lower amount of $\mathrm{C}_{\mathrm{org}}$ than those deposited during interglacial stages.

\section{DISCUSSION}

\section{Depositional Environment of Dark and Light Layers}

The observation that most of the dark (including faintly dark) and light layers are correlatable among the sites indicates that deposition of these layers is basinwide and nearly synchronous within the deeper part of the Japan Sea. The distance between Sites 797 and 795 (>700 $\mathrm{km}$ ) and slightly elevated altitudes above the basin floor at Sites 794, 795, and 797 (Tamaki, Pisciotto, Allan, et al., 1990) exclude the possibility of distal turbidite origin for most of the dark layers. Consequently, deposition of dark and light layers are thought to have been related to changes in paleoceanographic conditions in the Japan Sea.

\section{Bottom Water Oxygenation Level}

Examination of sedimentary structures shows that dark and faintly dark layers are either laminated or color-banded, whereas light layers are homogeneous, bioturbated, or color banded (Fig. 3). Preservation of lamina and color bands indicates deposition under anoxic condi- tions, whereas the presence of bioturbation suggests deposition under oxic conditions (Rhoads and Morse, 1971; Savrda and Bottjer, 1986).

Berner and Raiswell (1983) showed that the $\mathrm{C}_{\text {org }}$ to $\mathrm{S}_{\text {tot }}$ ratio (C/S) of fine-grained sediments from modern non-euxinic marine environments is more or less constant at approximately 3 , whereas those sediments deposited under euxinic conditions tend to have lower $\mathrm{C} / \mathrm{S}$ values. Euxinic conditions are defined here as depositional conditions with anoxic bottom water which contains dissolved $\mathrm{H}_{2} \mathrm{~S}$ (Berner and Raiswell, 1983). They suggested that the $\mathrm{C}_{\text {org }}$ vs. $\mathrm{S}_{\text {tot }}$ ratio can be used to distinguish euxinic from non-euxinic conditions.

Figure 10 shows the relationship between the $\mathrm{S}_{\text {tot }}$ and $\mathrm{C}_{\text {org }}$ for sediments with different sedimentary structures. Many laminated and color-banded samples, irrespective of their color rank, fall within the domain of euxinic conditions suggested by Berner and Raiswell (1983). But some of laminated and colorbanded samples fall within the non-euxinic domain. It is possible that pyrite formation in such samples was limited by the availability of reactive iron because of the dilution of detrital components by biogenic components (Berner, 1984). Samples 127-797B-2H-1, 102-104 cm, and 127-797B-4H-4, $135-137 \mathrm{~cm}$, which contain approximately 30 and $60 \mathrm{wt} \%$ of biogenic components, can be such examples. Other laminated and colorbanded samples within the non-euxinic domain, which do not contain $>30$ wt $\%$ biogenic components, probably represent non-euxinic anoxic conditions. This environment is defined as a depositional condition with almost zero dissolved oxygen and no dissolved $\mathrm{H}_{2} \mathrm{~S}$ within the bottom water.

Many homogeneous and bioturbated samples fall within the noneuxinic domain but some fall within the euxinic domain proposed by Berner and Raiswell (1983). However, such homogeneous or bioturbated samples that plot outside their non-euxinic domain still fall in the neighbor of the domain (Fig. 10). The figure also shows that the homogeneous samples which plot outside their non-euxinic domain fall in the same area as that of the bioturbated samples. As a consequence, it is not possible to distinguish depositional condition for the two groups of samples. We interpreted that homogeneous samples were also deposited under oxic conditions. Since the non-euxinic domain suggested by Berner and Raiswell (1983) is empirically defined and based on a limited number of samples, we slightly relaxed their boundary between the euxinic and non-euxinic domains so that all but one of our homogeneous and bioturbated samples fall within the non-euxinic domain (Fig. 10). The boundary is expressed as:

$$
\mathrm{S}_{\text {tot }}(\mathrm{wt} \%)=\mathrm{C}_{\mathrm{org}}(\mathrm{wt} \%) / 3+1 .
$$

The exception is Sample 127-797B-5H-3, 132-134 cm, which is homogeneous and still plots in the euxinic domain. It is not certain why this sample has an exceptionally low $\mathrm{C} / \mathrm{S}$ ratio although it does contain a small amount of volcanic glass.

By combining the data on sedimentary structures and the $\mathrm{C}_{\text {org }}$ versus $\mathrm{S}_{\text {tot }}$ relationship, it is possible to distinguish euxinic, non-euxinic-anoxic, and oxic depositional conditions. The result is illustrated in Figure 8L.

\section{Causes of Euxinic, Non-Euxinic-Anoxic, and Oxic Conditions}

Figure $8 \mathrm{~L}$ shows that glacial stages are generally characterized by euxinic to non-euxinic-anoxic conditions, whereas interglacial stages tend to be more oxic. An exception is glacial stage 8, during which the depositional condition appears rather oxic. Euxinic conditions also occurred during interglacial stages 5,7 , and 15 . Such euxinic events probably correspond to the lower sea-level periods represented by negative peaks of the $\delta^{18} \mathrm{O}$ curve.

Previous studies of piston cores from the Japan Sea indicated that a thick dark layer characterized by parallel lamination and a high pyrite content was deposited during the last glacial period (Oba, 1983, 1984; Masuzawa and Kitano, 1984; Oba et al., 1991). Oba (1983, 1984 ) and Oba et al. (1991) reported a decrease in the $\delta^{18} \mathrm{O}$ value of planktonic foraminifers by $3 \%$, in the uppermost part of this dark 
layer and interpreted this decrease as being the result of a lowering of surface water salinity. The lowering of the surface water salinity probably caused a density stratification and a reduction of the vertical mixing within the Japan Sea, thus resulting in basinwide euxinic conditions just like that in the present-day Black Sea (Oba, 1984; Masuzawa and Kitano, 1984). Based on the figures of Oba (1983, 1984), deposition of the dark layer took place from approximately 27 to $20 \mathrm{ka}$ and the excursion of the $\delta^{18} \mathrm{O}$ started at approximately $28 \mathrm{ka}$, reached the minimum between approximately 20 and $18 \mathrm{ka}$, and ended at approximately $16 \mathrm{ka}$.

Oba $(1983,1984)$ and Oba et al. (1991) further speculated that the lowering of the surface water salinity was due to the inflow of Yellow River water. Koizumi (1984) preferred the idea that the lowering of the surface water salinity was due to the isolation of the Japan Sea corresponding to a sea-level fall during the last glacial maximum and the consequent increase in the influence of the inflow of fresh water from rivers surrounding the Japan Sea. He also provided evidence that the abundance of low-salinity diatom species indicative of inflow from the Tsushima Strait was lowest during the period from 18.5 to $19.5 \mathrm{ka}$. Oba $(1984,1989)$ observed that the light $\delta^{18} \mathrm{O}$ peak occurred at approximately $20 \mathrm{ka}$, which is older than the age of the last glacial maximum (approximately $18 \mathrm{ka}$ ). For this time discrepancy, Oba prefers the inflow of Yellow River water, rather than isolation of the Japan Sea during the last glacial maximum, as a cause of the low salinity.

However, his age estimate of approximately $20 \mathrm{ka}$ for the light $\delta^{18} \mathrm{O}$ spike does not seem conclusive. Close examination of his figure (Oba, 1983; p. 41, Fig. 3) indicates that the light $\delta^{18} \mathrm{O}$ spike lasted from approximately 20 to $18 \mathrm{ka}$. Thus, we prefer the idea of Koizumi (1984) in that the low surface-water salinity event corresponded to the lowest stand of sea level during the last glacial maximum (oxygen isotopic event 2.2 ), which caused euxinic bottom water conditions and the deposition of the thick, laminated, pyrite-rich dark layer. This dark layer probably corresponds to the dark layer A in this study.

Non-euxinic-anoxic conditions occur both within glacial and interglacial stages but tend to coincide with periods when moderate $\delta^{18} \mathrm{O}$ values suggests moderate sea-level stands. Oba et al. (1991) suggested that the Yellow Sea and East China Sea water which is characterized by relatively low-salinity (approximately $30.5 \%$ o) flowed into the Japan Sea through the Tsushima Strait from 85 to $27 \mathrm{ka}$ when the warm Tsushima Current was absent. The inflow of this relatively low-salinity water was not sufficient enough to cause a strong density stratification and subsequent euxinic conditions, but might have inhibited generation of JSPW and caused non-euxinic-anoxic conditions.

Oxic conditions tend to occur during interglacial stages and coincide with periods characterized by high oxygen isotopic ratios. Oba et al. (1991) reported that a significant inflow of the warm Tsushima Current began at approximately $8 \mathrm{ka}$ when bottom water conditions turned oxic. The oxygenation of the bottom water was probably caused by an increase in surface water salinity and the consequent generation of JSPW.

Based on the previous discussion, correlation of the euxinic conditions with glacial lowstands observed at Site 797 is interpreted as being the result of isolation of the Japan Sea, subsequent lowering of surface water salinity, and the establishment of density stratification. On the other hand, the correlation of the oxic conditions with interglacial highstands is interpreted as being the result of a significant inflow of the warm Tsushima Current whose higher salinity enabled the generation of JSPW. Non-euxinic-anoxic conditions probably correspond to periods of intermediate sea-levels when the Japan Sea surface water was influenced by both the Yellow Sea and East China Sea waters. However, we need more supporting evidence, especially oxygen isotopic and paleontological data, to confirm this interpretation.

\section{Surface Productivity}

$\mathrm{C}_{\mathrm{org}}$ contents within pelagic and hemipelagic sediments are considered to reflect the productivity within the surface waters (e.g., Stein, 1991, and references therein). However, interpretation of the variation in the
$\mathrm{C}_{\mathrm{org}}$ content is not straightforward since the content is also affected by bottom water oxygenation levels and the input of terrigenous organic matter (Stein, 1991). The effect of these two factors may not be negligible in the upper Quaternary sediments of the Japan Sea.

The accumulation of biogenic silica, on the other hand, is less influenced by bottom water conditions and is used to infer surface water productivity (Broecker and Peng, 1982; Lisitzin, 1985). Biogenic silica contents in the upper Quaternary fine-grained sediments deposited in the Japan Sea during interglacial stages are higher than those deposited during glacial stages. Higher contents of biogenic silica result from either an increase in the mass accumulation rate (MAR) of biogenic silica or a decrease in the MAR of detrital materials. Although we do not have MAR data, changes in the linear sedimentation rate at Site 797 during the late Quaternary (based on our correlation described previously) shows that the linear sedimentation rates during glacial stages tend to be lower than those during interglacial stages (Fig. 11). This observation suggests that the higher biogenic silica contents during interglacials are due to an increase in the MAR of biogenic silica rather than a decrease in the MAR of detrital materials, which, in turn, suggests higher surface productivity during interglacials.

Based on the detailed study of three piston cores recovered from the eastern part of the Japan Sea, Koizumi (1989) discussed the interrelationship between diatom abundance and an inflow of the warm Tsushima Current into the Japan Sea during the last $10 \mathrm{ka}$. He showed that an increase in diatom abundance corresponds to a strong inflow of the Tsushima Current, especially in the southern part of the Japan Sea. He further suggests that a strong inflow of the Tsushima Current is caused by glacio-eustatic sea-level rises.

A paleoceanographic study by Oba et al. (1991) showed that an inflow of the Tsushima Current started after approximately $10 \mathrm{ka}$ and became significant after $8 \mathrm{ka}$. They observed an increase in the abundance of diatoms and a decrease in the $\delta^{13} \mathrm{C}$ value of benthic foraminifers relative to that of planktonic foraminifers after $8 \mathrm{ka}$, both indicating an increase in surface productivity. Global sea-levels were approximately 60 and 25 $\mathrm{m}$ lower than present at 10 and $8 \mathrm{ka}$, respectively (Fairbanks, 1989).

Considering that the tectonic subsidence in the eastern margin of the Tsushima Strait during the last $6 \mathrm{ka}$ is only $0-1.5 \mathrm{~m}$ (Ota and Machida, 1987), the depth of the Tsushima Strait during 10 to $8 \mathrm{ka}$ is estimated to have been $70-105 \mathrm{~m}$.

The evidence previously described suggests that surface productivity, which is reflected in the diatom content of the sediments (at least in the southern part of Japan Sea), was strongly influenced by the inflow of the warm Tsushima Current. The inflow of the Tsushima Current has been controlled dominantly by sea level, although the strength of the Kuroshio Current might also have affected the inflow (Chinzei et al., 1987). The reduced diatom content during glacials, on the other hand, might have been related to reduced nutrient supply from the deep water due to strong density stratification during glacial lowstands.

Deposition of thin $(<50 \mathrm{~cm})$ dark layers with high $\mathrm{C}_{\text {org }}$ content $(>1.5 \mathrm{wt} \%$ ) during periods of moderate sea-level stand is suggested as a result of this study. Some of such thin dark layers within isotopic stages $1,15,17,18$, and 20 (between 28 and 39 mbsf) are associated with high frequency occurrence of planktonic foraminifer Globigerina bulloides (Fig. 8F) (Brunner, this volume). High frequencies of G. bulloides in sediments are often used as an indicator of upwelling (Thiede, 1975, 1983; Prell and Curry, 1981; Wafer et al., 1983; Fischer et al., 1983; Ganssen and Sarnthein, 1983; Prell, 1984), but evidence from sediment trap studies (Thunell and Reynolds, 1984; Reynolds and Thunell, 1985; Sautter and Thunell, 1989, 1991) suggests that $G$. bulloides "proliferates in a variety of environmental conditions (in addition to upwelling), often as the dominant planktonic foraminifer" (Sautter and Thunell, 1991). We interpret high frequencies of $G$. bulloides tentatively as an upwelling indicator keeping in mind the caveat of Sautter and Thunell (1991) as stated above.

At Site 797 , the $\mathrm{C}_{\text {carb }}$ content tends to be enhanced within and proximal to such dark layers. The close association between high $\mathrm{C}_{\text {carb }}$ contents and/or high frequencies of $G$. bulloides in $\mathrm{C}_{\text {org }}$-rich thin dark 


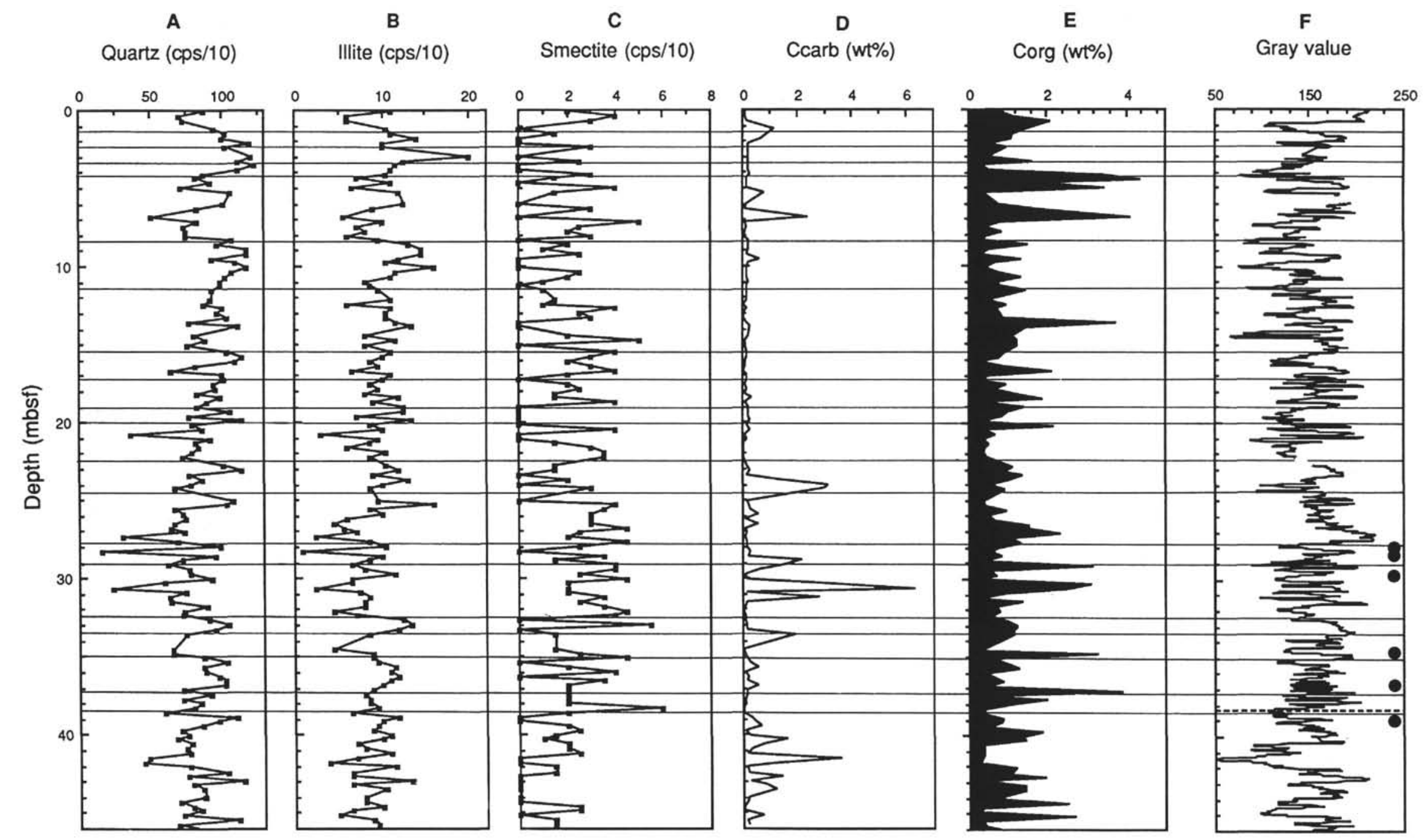

Figure 8. Vertical profiles of quartz peak height (A), illite peak height (B), smectite peak height (C), the Ccarb content (D)), the Corg content (E), gray value (F), the Stot content (G), the maximum size of detrital grains $(\mathbf{H})$, diatom content $(\mathbf{I})$, diatom number $(\mathbf{J})$, and the estimated bottom water oxygenation level $(\mathbf{L})$ for the samples from the upper Quaternary sediments at Site 797 in comparison with the standard oxygen isotope curve (K) by Imbrie et al. (1984). The oxygen isotope curve was interpolated between the five chronologically controlled levels (shown by arrows) described in the text. Horizontal lines in the figure represent boundaries of oxygen isotope stages. Also shown are the abundant occurrence of Globigerina bulloides (solid circles) on the gray value plot and the stratigraphic levels where dropstones occur at Site 795 (open diamonds) on the diatom number plot. 


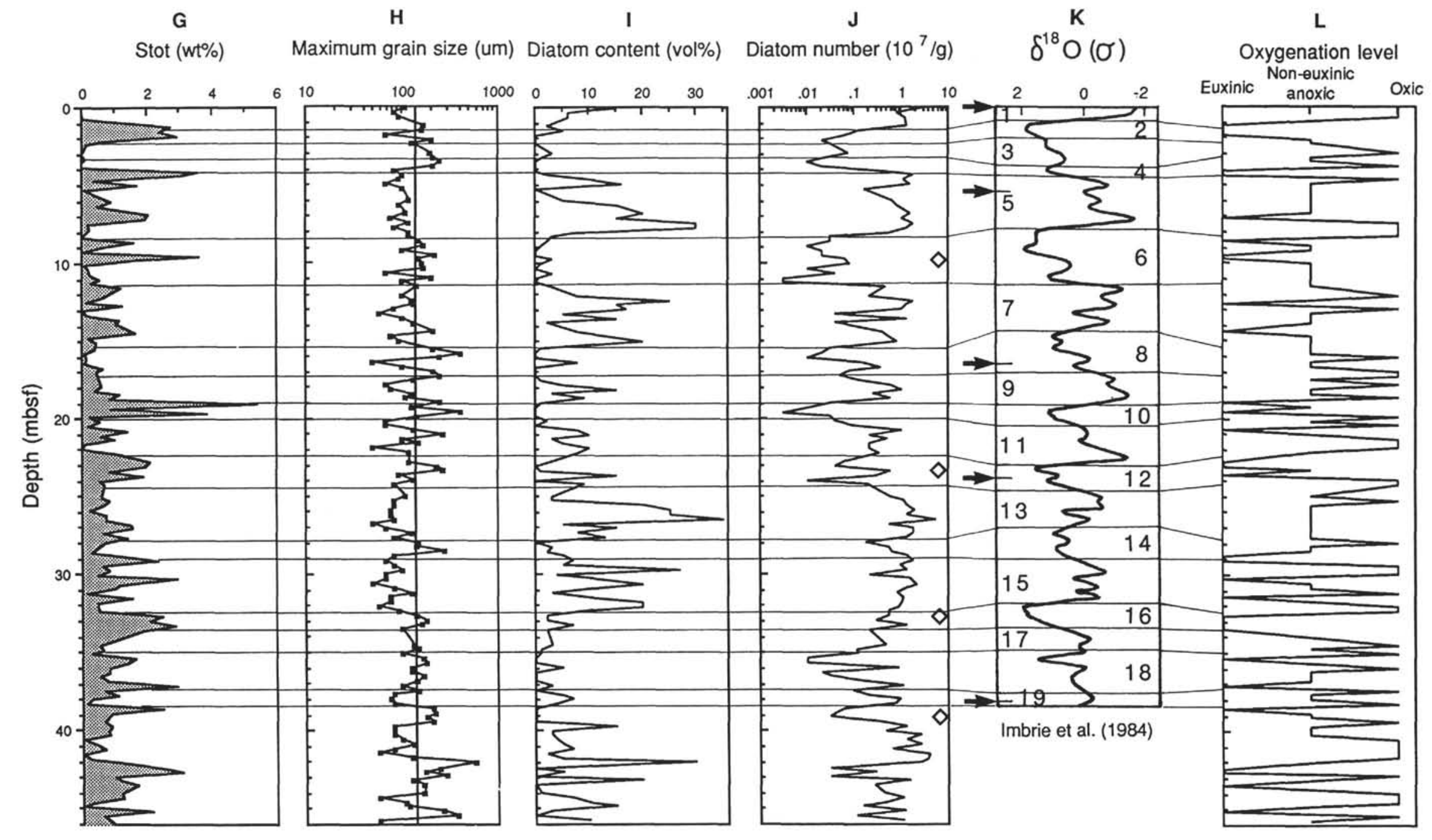

Figure 8 (continued). 


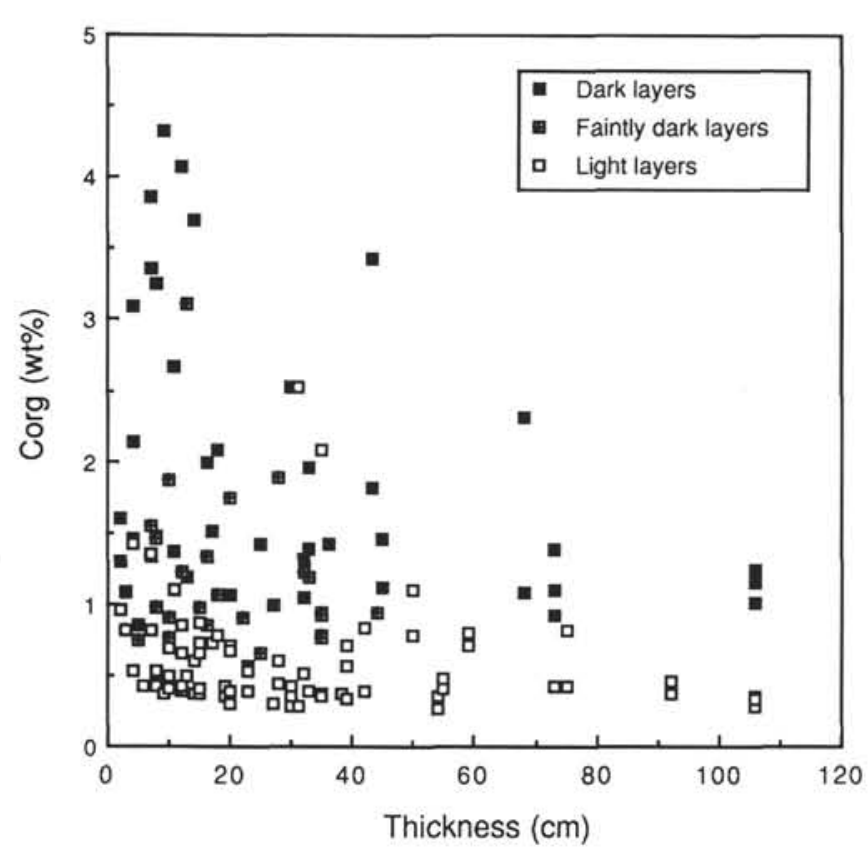

Figure 9 . The relationship between the $\mathrm{C}_{\mathrm{org}}$ content and thickness of the layers for samples from the upper Quaternary sediments at Site 797.

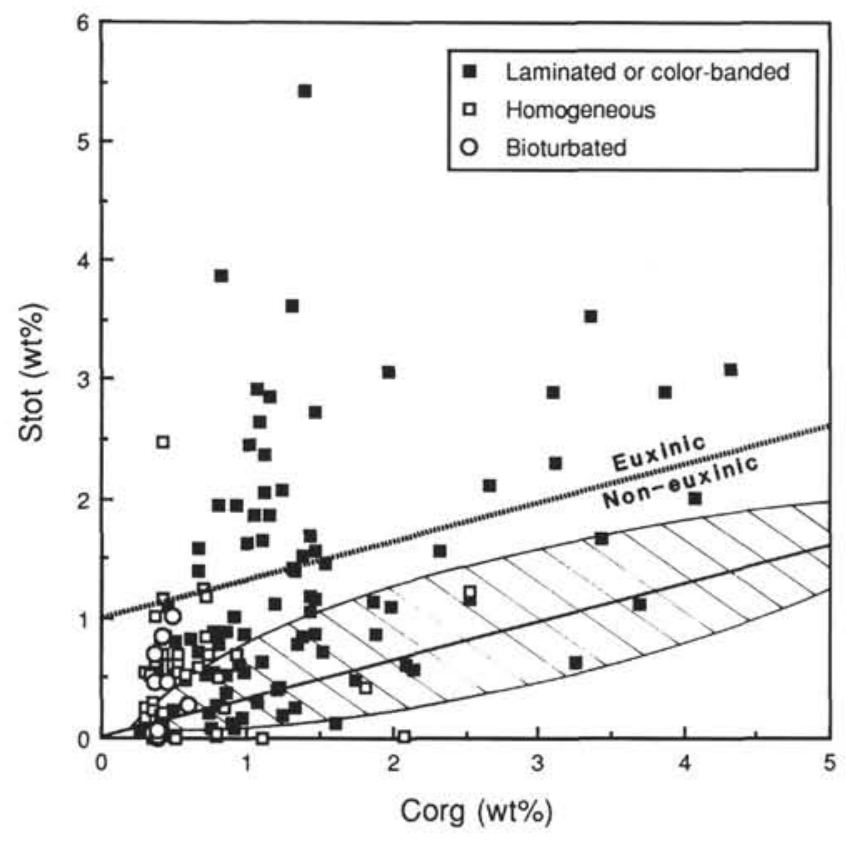

Figure 10. The relationship between the $\mathrm{S}_{\text {tot }}$ content and the $\mathrm{C}_{\mathrm{org}}$ content for samples from the upper Quaternary sediments at Site 797. The hatched area is the domain for normal marine sediments deposited under oxic conditions of Berner and Raiswell (1983). The dotted line is the boundary between euxinic and non-euxinic conditions proposed in this study.

layers, suggests that upwelling controlled deposition of the layers. Layers enriched in $\mathrm{C}_{\text {org }}$ are also closely associated with high $\mathrm{C}_{\text {carb }}$ and abundant calcareous microfossils in core $\mathrm{KH}-79-3, \mathrm{C}-3$ recovered from the Oki Ridge (Oba et al., 1991).

Oba et al. (1991) showed that deposition of the most recent thin $\mathrm{C}_{\text {org }}$-rich dark layer took place at approximately $11 \mathrm{ka}$ and was

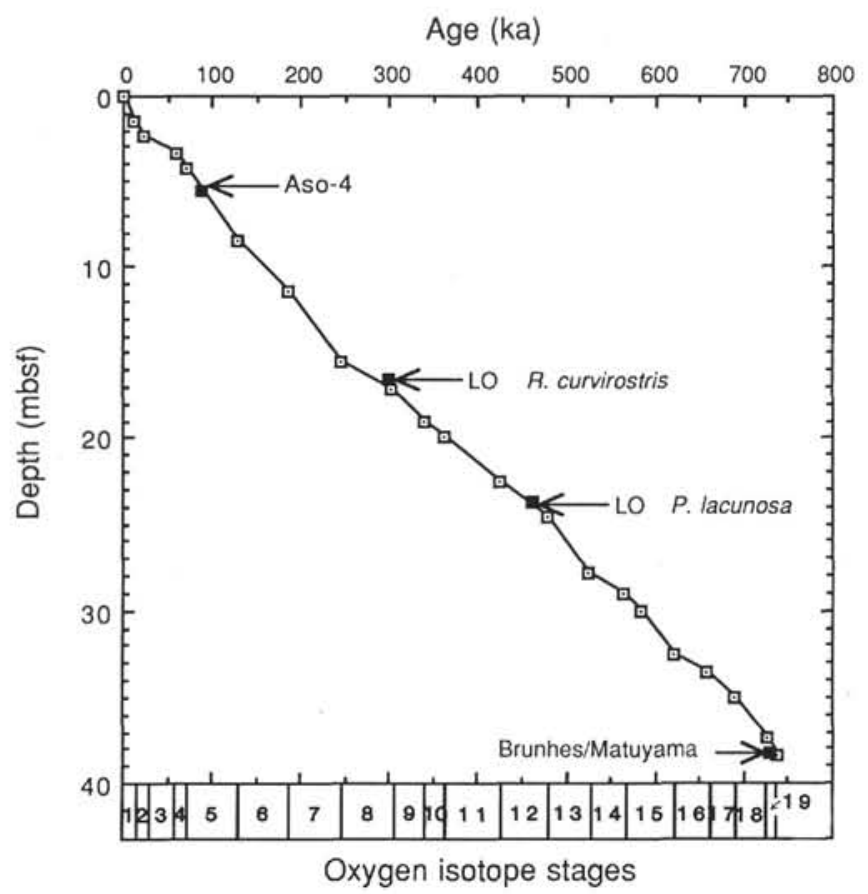

Figure 11. The sedimentation rate diagram for the upper Quaternary sediments of Site 797 based on the proposed correlation with the oxygen isotope curve of Imbrie et al. (1984). Solid squares are chronologically controlled points determined by other methods.

followed by a period of the bottom water conditions corrosive to carbonate between 10 and $8 \mathrm{ka}$. Oba and his colleagues interpreted that the two events were related to the cessation of inflow of the Oyashio Current and the initiation of inflow of the Tsushima Current to the Sea at approximately $11 \mathrm{ka}$. Such a switch of current systems may have caused conditions favorable for upwelling, which caused a bloom of calcareous plankton.

\section{Possible Origin of the Detrital Component}

The result of mineralogical analysis suggests that the detrital component of the upper Quaternary fine-grained sediments at Site 797 are characterized by the mineral assemblage of quartz $>$ plagioclase $>$ illite $=$ kaolinite plus chlorite $>$ smectite. Among these minerals, quartz, plagioclase, illite, and kaolinite plus chlorite have positive correlations, suggesting that they are behaving as one group. Smectite, on the other hand, is behaving independently. Tada and Iijima (this volume) obtained similar results for the Quaternary sediments from Sites 794 and 795.

Inoue and Naruse (1990) reported that the mineral composition of Chinese loess and modern eolian dusts in Japan are characterized by the assemblage of quartz $>$ plagioclase $>$ illite $=$ kaolinite. This assemblage is similar to that of the upper Quaternary sediments from Site 797 except for the absence of smectite within loess and eolian dusts. Mizota and Matsuhisa (1984), on the other hand, examined the oxygen isotope composition of detrital quartz extracted from the uppermost Quaternary sediments of the Japan Sea. They showed that the oxygen isotopic composition of the detrital quartz is close to the value of typical eolian dust in Japan, and concluded that the detrital quartz within the sediments is dominantly of eolian origin. Moreover, Uematsu et al. (1983) estimated the modern accumulation rate of eolian dust on the Japan Sea side of Japanese Islands at 1.4 to 4.3 $\mathrm{g} / \mathrm{cm}^{2} / 1000 \mathrm{yr}$. These rates are comparable with the average MAR of $4.6 \mathrm{~g} / \mathrm{cm}^{2} / 1000 \mathrm{yr}$ for the upper Quaternary sediments at Site 797 (Tamaki, Pisciotto, Allan, et al., 1990). 
It is possible that these detrital minerals were supplied by the Yellow River, which has a sediment load similar in mineral composition to that of the loess (Yin et al., 1987). However, other lines of evidence tend to indicate that a significant portion of the detrital component (except smectite) in the upper Quaternary sediments of the Japan Sea is of eolian origin although a supply from the Yellow River cannot be excluded, especially during glacial periods.

Smectite, on the other hand, tends to be enriched in the offshore sediments of the northeastern Japan Sea and is considered to be supplied from the northern part of the Japanese Islands (Yin et al., 1987). The observation that the detrital minerals (except smectite) tend to be enriched in sediments deposited during glacial stages could be interpreted as a result of enhanced eolian input and/or inflow of the Yellow River during glacial periods. Whereas the observation that smectite tends to be enriched in sediments deposited during interglacial stages can be interpreted as a result of reduced input of the detrital minerals (except smectite) and a consequent increase in relative importance of smectite supplied from northern Japan during interglacial periods.

Our results also show that the maximum grain size tends to be larger during glacial stages rather than during interglacial stages. This can be interpreted as a result of stronger wind intensities or closer source areas (Janecek and Rea, 1985) if we accept the eolian origin for the detrital silt. Whereas, it could be interpreted as a result of stronger inflow of the Yellow River into the Japan Sea, if we consider the Yellow River as a source of the detrital material. We need more investigation to specify the origin of detrital materials.

\section{SUMMARY}

Inspection of gray value profiles and core photographs has revealed that most of the dark and light layers found in the upper Quaternary sediments of the Japan Sea are correlatable among Sites 794, 795, and 797. Marker ash layers provide a parallelism with adjacent dark layers. This observation suggests that the deposition of dark and light layers reflects basinwide, approximately synchronous events.

At Site 797, sediment ages were controlled at three levels between the Recent and the Brunhes/Matuyama boundary. The age of sediments deposited between these marker levels was estimated by linear interpolation. Examination of the variation in the sediment composition with age suggests that it is closely related to glacial-interglacial cycles most commonly expressed in the standard oxygen isotope curve (Imbrie et al., 1984). In particular, the abundance of diatoms shows the age variation pattern in a similar manner to that of the oxygen isotope curve. The latter information enabled us to "tune" the age of the sediment with well-established oxygen isotope stages.

Based on the "tuned" ages, detrital minerals such as quartz, plagioclase, illite, and kaolinite plus chlorite tend to be enriched and the maximum grain size of detrital silts tends to be larger during glacial stages, whereas biogenic silica and smectite tend to be enriched and the maximum grain size tends to be reduced during interglacial stages. $\mathrm{S}_{\text {tot }}$ tends to be enriched in thick dark layers deposited during glacial stages, whereas $\mathrm{C}_{\text {org }}$ tends to be enriched in the thin dark layers which were deposited during interglacial stages with moderate sea-level stands.

The bottom water oxygenation level was estimated by combining the evidence of the $\mathrm{C}_{\text {org }} / \mathrm{S}_{\text {tot }}$ ratios and the sedimentary structures. The results suggest that bottom water tends to have been euxinic during glacial lowstands and oxic during interglacial highstands. The euxinic conditions were probably caused by reduced vertical mixing as a result of a strong density stratification, which, in turn, was caused by a lowering of the surface water salinity due to isolation of the Japan Sea during glacial lowstands and subsequent inflow of fresh waters. The oxic conditions, on the other hand, were probably related to a strong inflow of the warm Tsushima Current and subsequent enhance- ment of production of JSPW during interglacial highstands. During intermediate sea-level stands, non-euxinic-anoxic conditions prevailed and thin $\mathrm{C}_{\mathrm{org}}$-rich dark layers were deposited. The depositional periods may correspond to periodic upwelling events which might have been related to the intermittent inflow of the Tsushima Current to the Japan Sea.

The detrital minerals such as quartz, plagioclase, illite, and kaolinite plus chlorite are interpreted as eolian in origin. However, the possibility that they were supplied from the Yellow River cannot be excluded. If the former is the case, their deposition reflects aridity and proximity of the source area(s) and the wind intensity. If the latter is the case, their deposition reflects degree of inflow of the Yellow River, which might have been closely related to sea level.

These results imply that the Japan Sea has behaved as a single sedimentary system which responds to climatic changes, especially glacio-eustatic sea-level changes. The sedimentary record could provide high-resolution information on climate and paleoceanography during the Quaternary.

\section{ACKNOWLEDGMENTS}

We express our special thanks to Dr. C. Brunner for her many useful suggestions and critical reading of the manuscript. We also thank Dr. K. Tanabe for the use of his graphic analyzer. This study is partly supported by the Grant-in-Aid for Scientific Research of the Ministry of Education, Science and Culture of Japan No. 6247005 provided to $\mathrm{R}$. Tada.

\section{REFERENCES}

Berner, R. A., 1971. Principles of Chemical Sedimentology: New York (McGraw-Hill).

1984. Sedimentary pyrite formation: An update. Geochim. Cosmochim. Acta, 48:605-615.

Berner, R. A., and Raiswell, R., 1983. Burial of organic carbon and pyrite sulfur in sediments over Phanerozoic time: a new theory. Geochim. Cosmochim. Acta, 47:855-862.

Broecker, W. S., and Peng, T.-H., 1982. Tracers in the Sea: New York (Eldigio Press).

Chinzei, K., Fujioka, K., Kitazato, H., Koizumi, I., Oba, T., Oda, M., Okada, H., Sakai, T., and Tanimura, Y., 1987. Postglacial environmental change of the Pacific Ocean off the coasts of central Japan. Mar. Micropaleontol., 11:273-291.

Fairbanks, R. G., 1989. A 17,000-year glacio-eustatic sea level record: influence of glacial melting rates on the Younger Dryas event and deep-ocean circulation. Nature, 342:637-642.

Fischer, K., Dymond, J., Moser, C., Murray, D., and Mathern, A., 1983. Seasonal variation in particulate flux in an offshore area adjacent to coastal upwelling. In Suess, E., and Thiede, J., (Eds.), Coastal Upwelling: Its Sediment Record (Pt. A): New York (Plenum), 209-244.

Fukuoka, J., 1962. Characteristics of hydrography of the Japan Sea-in comparison with hydrography of the North Pacific. J. Oceanogr. Soc. Jpn., 20:180-188. (in Japanese with English abstract)

Gamo, T., and Horibe, Y., 1983. Abyssal circulation in the Japan Sea. J. Oceanogr. Soc. Jpn., 39:220-230.

Gamo, T., Nozaki, Y., Sakai, H., Nakai, T., and Tsubota, H., 1986. Spacial and temporal variations of water characteristics in the Japan Sea bottom layer. J. Mar. Res., 44:781-793.

Ganssen, G., and Sarnthein, M., 1983. Stable-isotope composition of foraminfers: the surface and bottom water record of coastal upwelling. In Suess, E., and Thiede, J. (Eds.), Coastal Upwelling: Its Sediment Record (Pt. A): New York (Plenum), 99-121.

Hidaka, K., 1966. Japan Sea. In Fairbridge, R. W. (Ed.), Encyclopedia of Oceanography: New York (Reinhold), 417-424.

Ichikura, M., and Ujiie, H., 1976. Lithology and planktonic foraminifera of the Sea of Japan piston cores. Bull. Nat. Sci. Mus. Ser. C (Geology), 2:151-178.

Imbrie, J., Shackleton, N. J., Pisias, N. G., Morley, J. J., Prell, W. L., Martinson, D. G., Hays, J. D., McIntyre, A., and Mix, A. C., 1984. The orbital theory 
of Pleistocene climate: Support from revised chronology of the marine $\delta^{18} \mathrm{O}$ record. In Berger, A., Imbrie, J., Hays, J., Kukla, G., and Saltzman, B. (Eds.), Milankovitch and Climate: Dordrecht (Riedel), 269-305.

Inoue, K., and Naruse, T., 1990. Asian long-range eolian dust deposited on soils and paleosols along the Japan Sea Coast. Quat. Res., 29:209-222. (in Japanese with English abstract)

Janecek, T. R., and Rea, D. K., 1985. Quaternary fluctuation in the Northem Hemisphere tradewinds and westerlies. Quat. Res., 24:150-163.

Kobayashi, K., 1985. Sea of Japan and Okinawa Trough. In Nairn, A.E.M., Stehli, F., and Uyeda, S. (Eds.), The Ocean Basins and Margins (Vol. 7A): The Pacific Ocean: New York (Plenum), 419-458.

Koizumi, I., 1984. Diatoms-analysis of KH-79-3, C-3 core. Chikyu Monthly, 6:547-553. (in Japanese)

, 1989. Holocene pulses of diatom growths in the warm Tsushima Current in the Japan Sea. Diatom Res., 4:55-68.

Lisitzin, A. P., 1985. The silica cycle during the last ice age. Palaeogeogr., Palaeoclimatol., Palaeoecol., 50:241-270.

Machida, H., 1987. Comments on the widespread tephras of Japan. Quat. Res., 25:265-268. (in Japanese with English abstract)

Masuzawa, T., and Kitano, Y., 1984. Appearance of $\mathrm{H}_{2} \mathrm{~S}$-bearing bottom waters during the last glacial period in the Japan Sea. Geochem. J., 18:167-172.

Mizota, C., and Matsuhisa, Y., 1984. Eolian dust-mainly based on analysis of core KH-79-3, C-3. Chikyu Monthly, 6:553-557. (in Japanese)

Moriyasu, S., 1972. The Tsushima Current. In Stommel, H., and Yoshida, K. (Eds.), Kuroshio, its Physical Aspects: Tokyo (Univ. Tokyo Press), 353-369.

Oba, T., 1983. Paleoenvironment of the Japan Sea since the last glacial period. Chikyu Monthly, 5:37-46. (in Japanese)

, 1984. Oxygen and carbon isotopic ratios-analysis of $\mathrm{KH}-79-3$, C-3 core. Chikyu Monthly, 6:558-566. (in Japanese)

, 1989. Environmental change in the Japan Sea-history of the last 85,000 years. Kagaku, 59:672-681. (in Japanese)

, 1991. Stratigraphic positions of Aso-4 and Ata tephras based on oxygen isotope stratigraphy. Chikyu Monthly, 13:224-227. (in Japanese)

Oba, T., Kato, M., Kitazato, H., Koizumi, I., Omura, A., Sakai, T., and Takayama, T., 1991. Paleoenvironmental changes in the Japan Sea during the last 85,000 years. Paleoceanography, 6:499-518.

Oba, T., Omura, A., Kato, M., Kitazato, H., Koizumi, I., Sakai, T., Takayama, T., and Mizota T., 1984. History of paleoenvironmental changes-analysis of KH-79-3, C-3 core. Chikyu Monthly, 6:571-575. (in Japanese)

Ogihara, S., and Iijima, A., 1991. Determination of total sulfur content of sedimentary rocks by a iodometric titration method. J. Jpn. Assoc. Min. Petr. Econ. Geol., 86:112-115.

Ohwada, M., and Tanioka, K., 1971. Cruise report on the simultaneous observation of the Japan Sea in October 1969. Oceanogr. Mag., 23:47-58.

Ota, Y., and Machida, H., 1987. Quaternary sea-level changes in Japan. In Tooley, M., and Shennan, I. (Eds.), Sea-level Changes: New York (Blackwell), 182-224.

Potter, P. E., Maynard, J. B., and Pryor, W. A., 1980. Sedimentology of Shale: New York (Springer).

Prell, W., 1984. Variation of monsoonal upwelling: a response to changing solar radiation. In Hansen, J. E., and Takahashi, T., (Eds.), Climatic Process and Climate Sensitivity: Am. Geophys. Union Monogr., 29:48-57.

Prell, W., and Curry, W. B., 1981. Faunal and isotopic indices of monsoonal upwelling: Western Arabian Sea. Oceanol. Acta, 4:91-98.

Reynolds, L., and Thunell, R. C., 1985. Seasonal succession of planktonic foraminifera in the subpolar North Pacific. J. Foraminiferal Res., 15:282-301.
Rhoads, D. C., and Morse, J. W., 1971. Evolutionary and ecologic significance of oxygen-deficient marine basins. Lithaia, 4:413-428.

Sautter, L. R., and Thunell, R. C., 1989. Seasonal succession of planktonic foraminifera: results from a four-year time-series sediment trap experiment in the northeast Pacific. J. Foraminiferal Res., 19:253-267. , 1991. Planktonic foraminiferal response to upwelling and seasonal hydrographic conditions: sediment trap results from San Pedro Basin, Southern California Bight. J. Foraminiferal Res., 21:347-363.

Savrda, C. E., and Bottjer, D. J., 1986. Trace-fossil model for reconstruction of paleo-oxygenation in bottom waters. Geology, 14:3-6.

Stein, R., 1991. Accumulation of Organic Carbon in Marine Sediments: Berlin (Springer).

Tada, R., 1991. Origin of rhythmical bedding in middle Miocene siliceous rocks of the Onnagawa Formation, northern Japan. J. Sediment. Petrol., 61:1123-1145.

Tamaki, K., Pisciotto, K., Allan, J., et al., 1990. Proc. ODP, Init. Repts., 127: College Station, TX (Ocean Drilling Program).

Tanaka, T., in press. Sediments and sedimentary processes on the continental slope and deep basin in the Japan Sea. In Isezaki, N., et al. (Eds.), Geology and Geophysics of the Japan Sea. Japan-USSR Monogr. Ser., 1.

Thiede, J., 1975. Distribution of foraminifera in surface waters of a coastal upwelling area. Nature, 253:712-714.

, 1983. Skeletal plankton and nekton in upwelling water masses off northwestern South America and northwestern Africa. In Suess, E., and Thiede, J. (Eds.), Coastal Upwelling: Its Sedimentary Record (Pt. A): New York (Plenum), 183-208.

Thierstein, H. R., Geltzenauer, K. R., Molfino, B., and Shackleton, N. J., 1977. Global synchroneity of late Quaternary coccolith datum levels: validation by oxygen isotopes. Geology, 5:400-404.

Thunell, R. C., and Reynolds, L., 1984. Sedimentation of planktonic foraminifera: seasonal changes in species flux in the Panama Basin. Micropaleontology, 30:341-260.

Uematsu, M., Duce, R. A., Prospero, J. M., Chen, L., Merrill, J. T., and McDonald, R. L., 1983. Transport of mineral aerosol from Asia over the North Pacific Ocean. J. Geophys. Res., 88:5343-5352.

Ujiie, H., and Ichikura, M., 1973. Holocene-uppermost Pleistocene planktonic foraminifers of a piston core from off San'in district, Sea of Japan. Trans. Proc. Palaeontol. Soc. Jpn., 91:137-150.

Wafer, G., Dunbar, R. B., and Suess, E., 1983. Stable isotopes of foraminifers off Peru recording high fertility and changes in upwelling history. In Suess, E., and Thiede, J. (Eds.), Coastal Upwelling: Its Sedimentary Record (Pt. A): New York (Plenum), 295-308.

Yin, J., Okada, H., and Labeyrie, L., 1987. Clay mineralogy of slope sediments around Japanese Islands. Geosci. Rep. Shizuoka Univ., 13:41-65. (in Japanese with English abstract)

Date of initial receipt: 20 March 1991

Date of acceptance: 24 January 1992

Ms 127/128B-160 
1

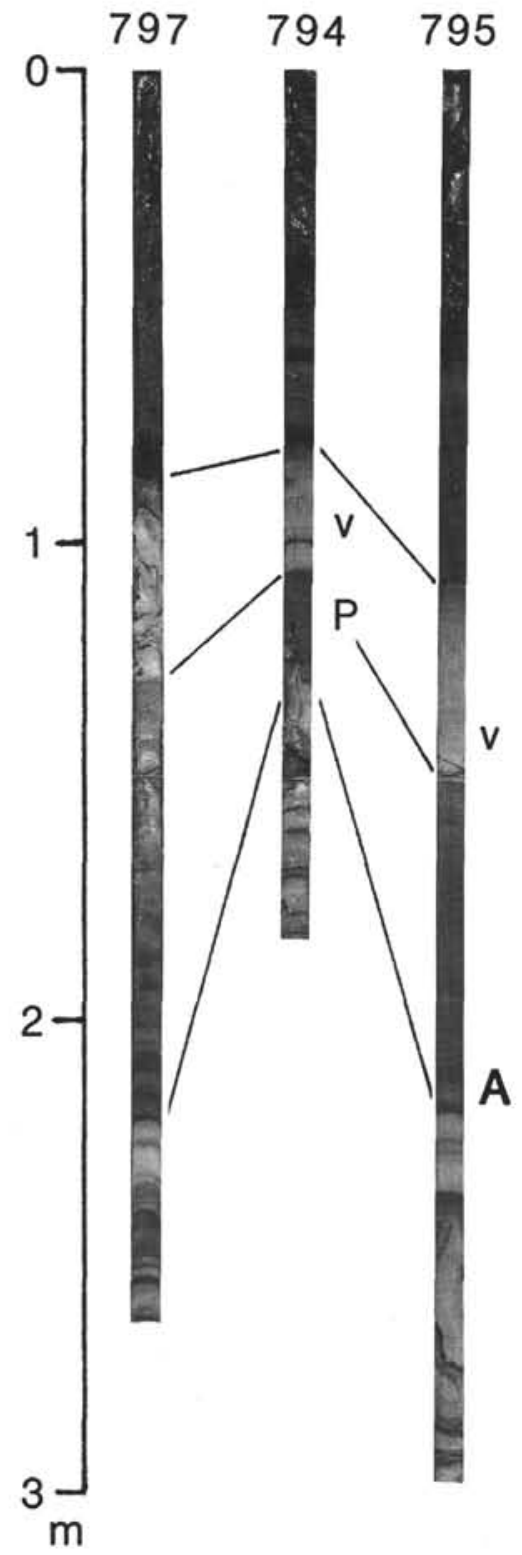

2

$\begin{array}{lll}797 & 794 & 795\end{array}$
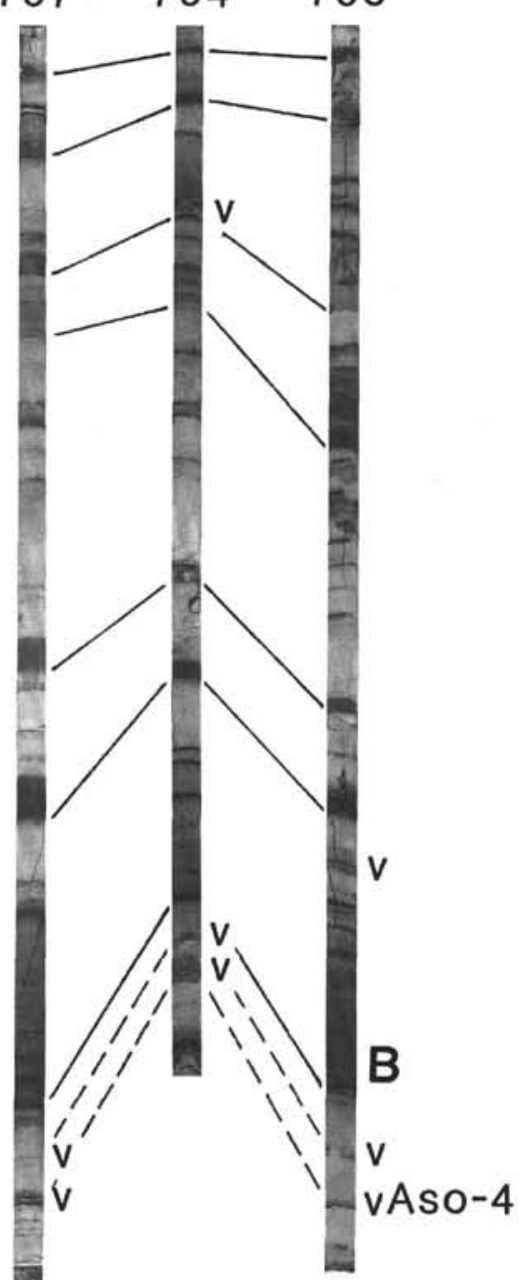

$\begin{array}{lll}797 & 794 & 795\end{array}$

Plates 1 through 5. Correlation of dark layers within the upper Quaternary sediments among Sites 794, 795, and 797. The labels A through $\mathrm{M}$ for the thick dark layers correspond to those in Figures 2 and 4 . The positions of Aso-4, $\mathrm{T}_{1}$ and other ash layers (v), dropstones (diamond), and plant fragments $(\mathrm{P})$ are also shown.

Plate 1. 1. Intervals from 797B-1H-1, $0 \mathrm{~cm}$, to $797 \mathrm{~B}-1 \mathrm{H}-2,150 \mathrm{~cm}, 794 \mathrm{~A}-1 \mathrm{H}-1,0 \mathrm{~cm}$, to $794 \mathrm{~A}-1 \mathrm{H}-2,34 \mathrm{~cm}$, and $795 \mathrm{~A}-1 \mathrm{H}-1,0 \mathrm{~cm}$, to $795 \mathrm{~A}-1 \mathrm{H}-2,150 \mathrm{~cm}$. 2. Intervals from 797B-1H-3, $0 \mathrm{~cm}$, to $797 \mathrm{~B}-1 \mathrm{H}-\mathrm{CC}, 20 \mathrm{~cm}, 794 \mathrm{~A}-1 \mathrm{H}-2,34 \mathrm{~cm}$, to $794 \mathrm{~A}-1 \mathrm{H}-3,110 \mathrm{~cm}$, and $795 \mathrm{~A}-1 \mathrm{H}-3,0 \mathrm{~cm}$, to $795 \mathrm{~A}-1 \mathrm{H}-4,115 \mathrm{~cm}$. 3. Intervals from $797 \mathrm{~B}-2 \mathrm{H}-1,0 \mathrm{~cm}$, to $797 \mathrm{~B}-2 \mathrm{H}-3,10 \mathrm{~cm}, 794 \mathrm{~A}-1 \mathrm{H}-3,110 \mathrm{~cm}$, to $794 \mathrm{~A}-1 \mathrm{H}-5,50 \mathrm{~cm}$, and $795 \mathrm{~A}-1 \mathrm{H}-4,115 \mathrm{~cm}$, to $795 \mathrm{~A}-1 \mathrm{H}-6,60 \mathrm{~cm}$. 
1

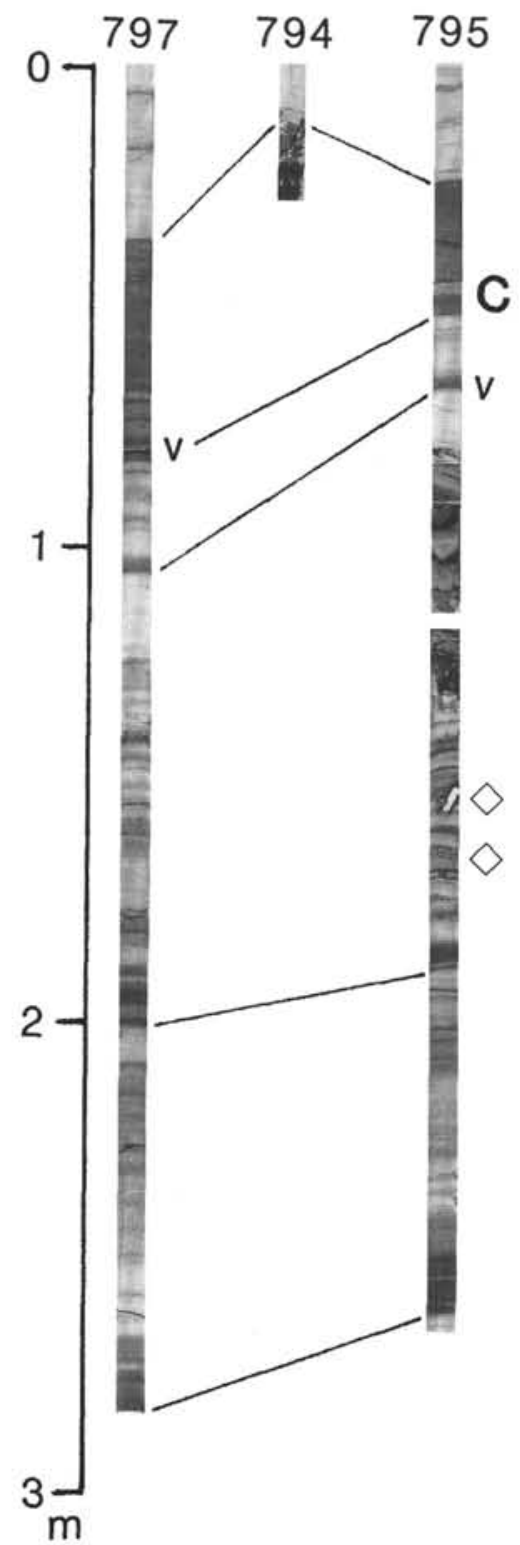

2

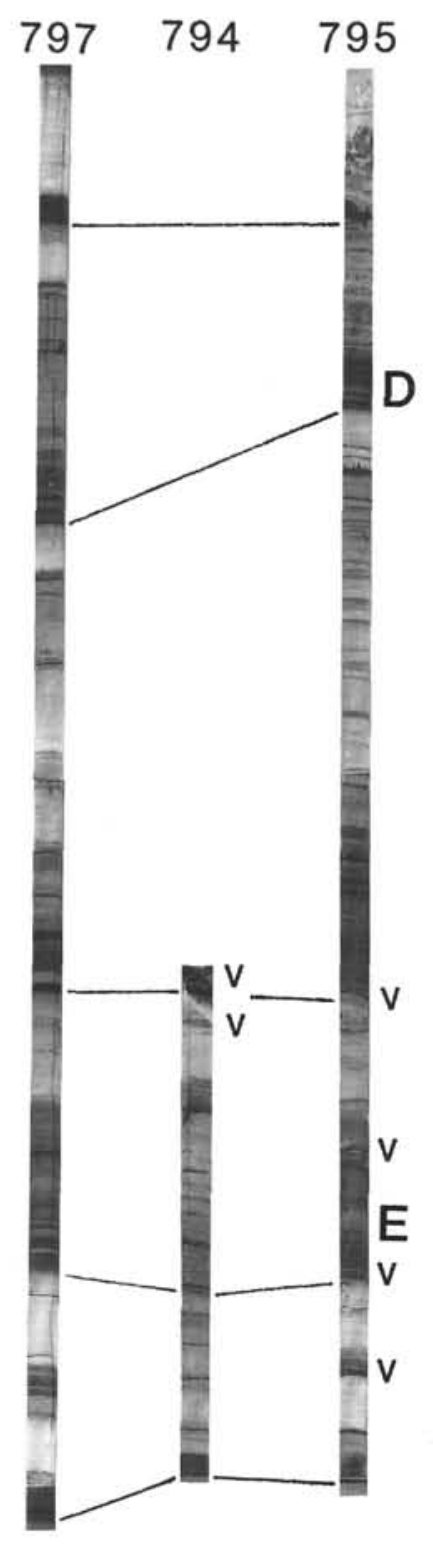

3

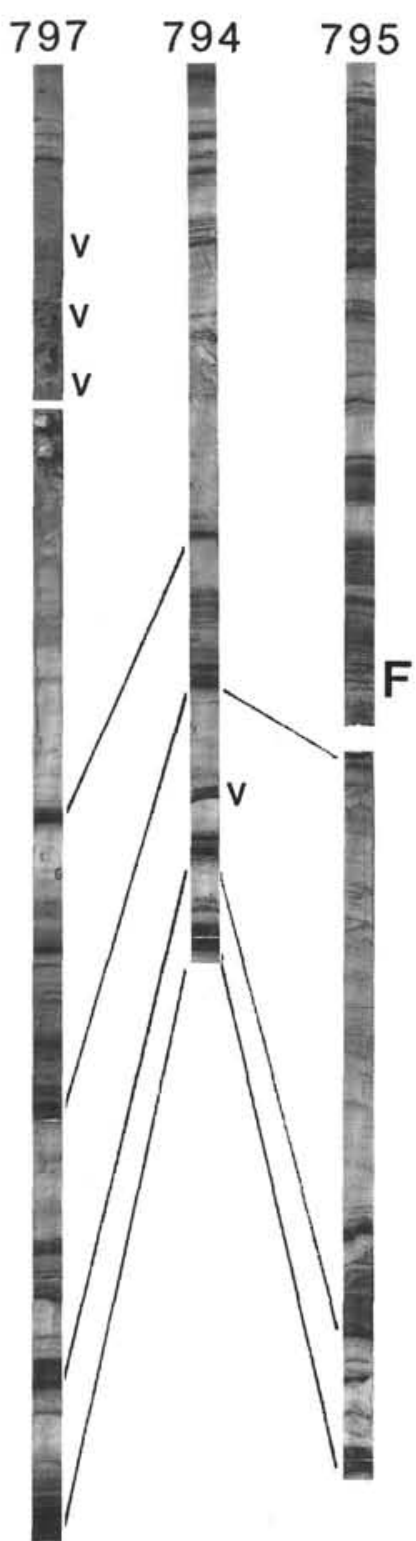

Plate 2. 1. Intervals from 797B-2H-3, $10 \mathrm{~cm}$, to $797 \mathrm{~B}-2 \mathrm{H}-4,150 \mathrm{~cm}, 794 \mathrm{~A}-1 \mathrm{H}-5,50 \mathrm{~cm}$, to $794 \mathrm{~A}-1 \mathrm{H}-\mathrm{CC}, 20 \mathrm{~cm}$, and $795 \mathrm{~A}-1 \mathrm{H}-6,60 \mathrm{~cm}$, to $795 \mathrm{~A}-2 \mathrm{H}-1,150 \mathrm{~cm}$. 2. Intervals from 797B-2H-5, $0 \mathrm{~cm}$, to 797B-2H-7, $10 \mathrm{~cm}, 794 \mathrm{~A}-2 \mathrm{H}-1,0 \mathrm{~cm}$, to $794 \mathrm{~A}-2 \mathrm{H}-1,110 \mathrm{~cm}$, and $795 \mathrm{~A}-2 \mathrm{H}-2,0 \mathrm{~cm}$, to $795 \mathrm{~A}-2 \mathrm{H}-4,5 \mathrm{~cm}$. 3. Intervals from $797 \mathrm{~B}-2 \mathrm{H}-7,10 \mathrm{~cm}$, to $797 \mathrm{~B}-3 \mathrm{H}-2,91 \mathrm{~cm}, 794 \mathrm{~A}-2 \mathrm{H}-1,10 \mathrm{~cm}$, to $794 \mathrm{~A}-2 \mathrm{H}-3,5 \mathrm{~cm}$, and 795A-2H-4, $5 \mathrm{~cm}$, to $795 \mathrm{~A}-2 \mathrm{H}-6,5 \mathrm{~cm}$. 
1

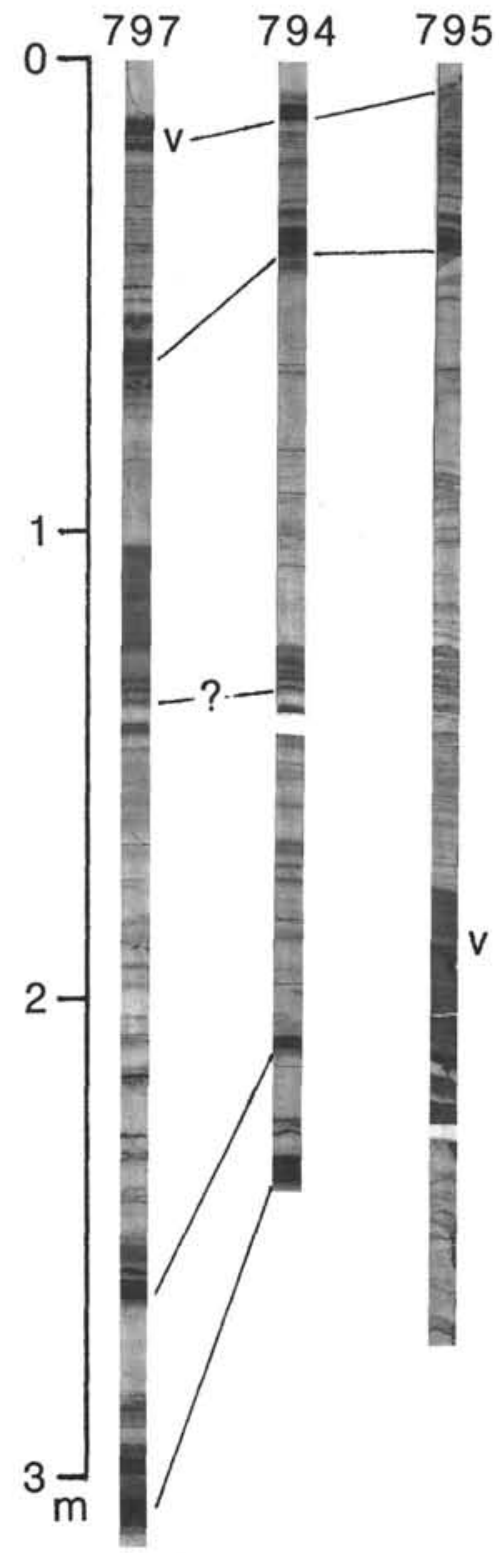

2

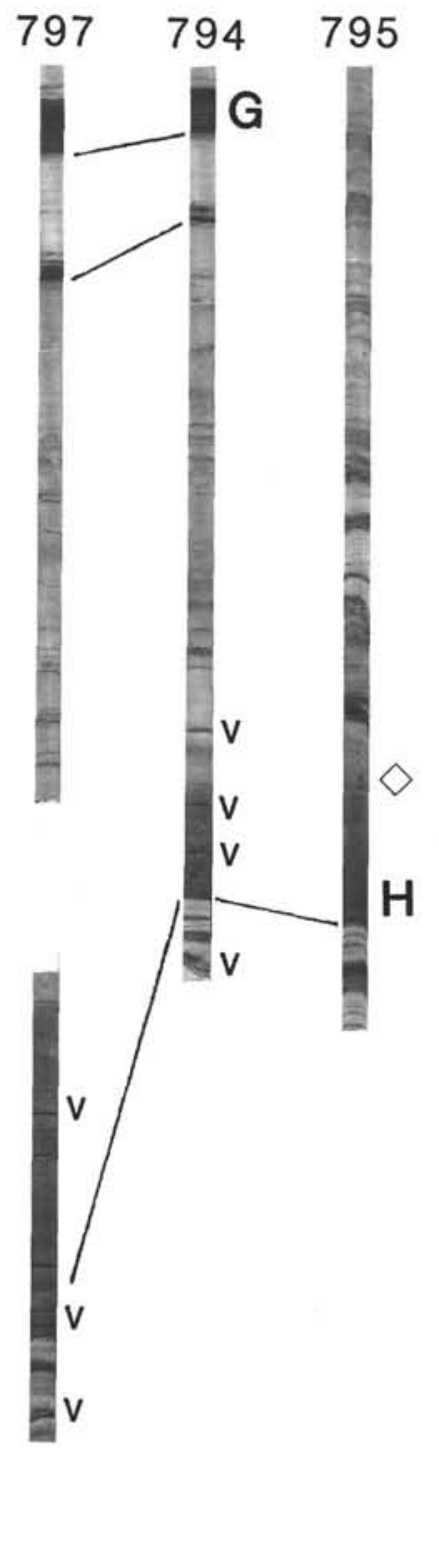

3

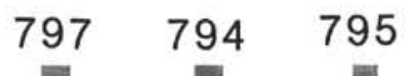

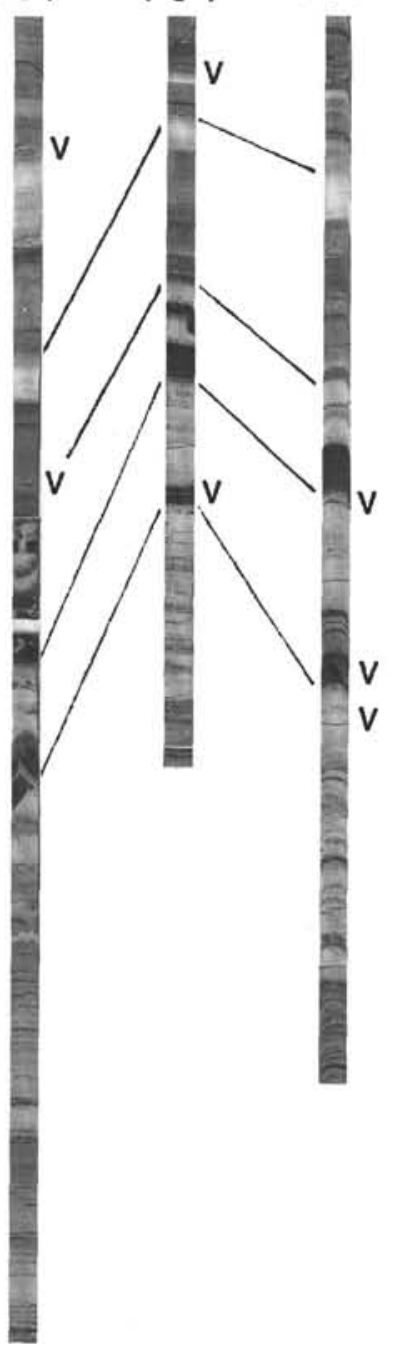

Plate 3. 1. Intervals from 797B-3H-2, $91 \mathrm{~cm}$, to $797 \mathrm{~B}-3 \mathrm{H}-4,107 \mathrm{~cm}, 794 \mathrm{~A}-2 \mathrm{H}-3,5 \mathrm{~cm}$, to $794 \mathrm{~A}-2 \mathrm{H}-4,97 \mathrm{~cm}$, and $795 \mathrm{~A}-2 \mathrm{H}-6,5 \mathrm{~cm}$, to $795 \mathrm{~A}-3 \mathrm{H}-1,45 \mathrm{~cm}$. 2 . Intervals from 797B-3H-4, $107 \mathrm{~cm}$, to 797B-3H-6, $98 \mathrm{~cm}, 794 \mathrm{~A}-2 \mathrm{H}-4,97 \mathrm{~cm}$, to $794 \mathrm{~A}-2 \mathrm{H}-5,144 \mathrm{~cm}$, and $795 \mathrm{~A}-3 \mathrm{H}-1,45 \mathrm{~cm}$, to $795 \mathrm{~A}-3 \mathrm{H}-2,95 \mathrm{~cm}$. 3. Intervals from $797 \mathrm{~B}-3 \mathrm{H}-6,98 \mathrm{~cm}$, to $797 \mathrm{~B}-4 \mathrm{H}-\mathrm{l}, 150 \mathrm{~cm}, 794 \mathrm{~A}-2 \mathrm{H}-5,144 \mathrm{~cm}$, to $794 \mathrm{~A}-2 \mathrm{H}-7,7 \mathrm{~cm}$, and $795 \mathrm{~A}-3 \mathrm{H}-2,95 \mathrm{~cm}$, to $795 \mathrm{~A}-3 \mathrm{H}-4,30 \mathrm{~cm}$. 
1

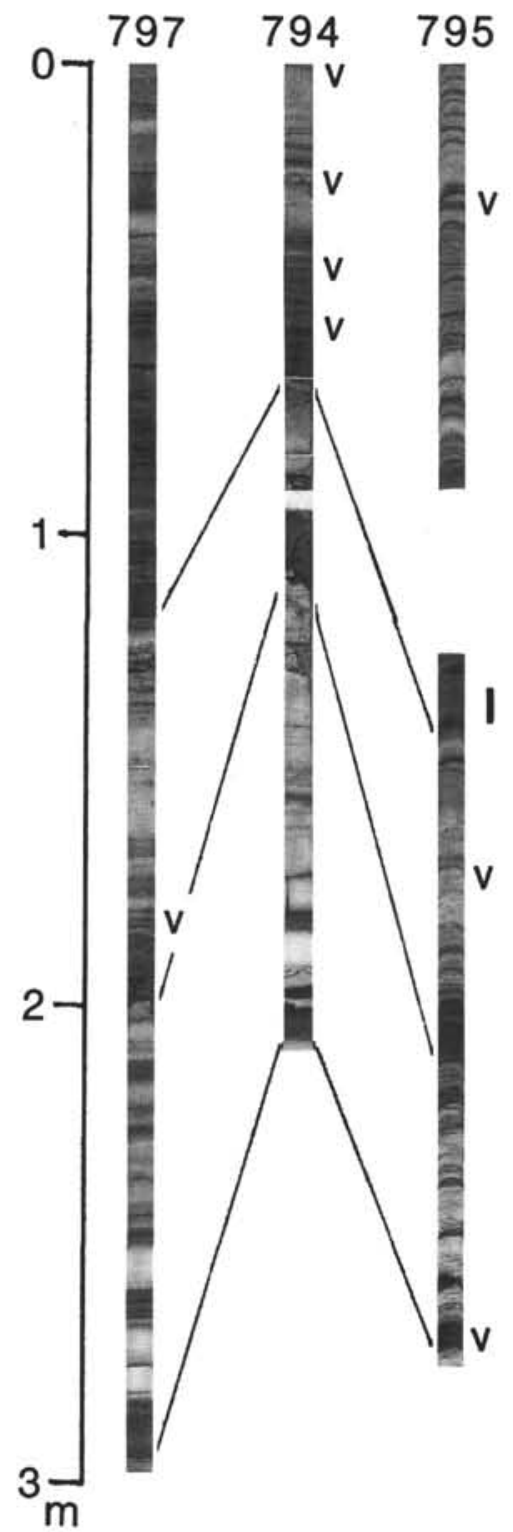

2

$\begin{array}{llll}797 & 794 & 795\end{array}$
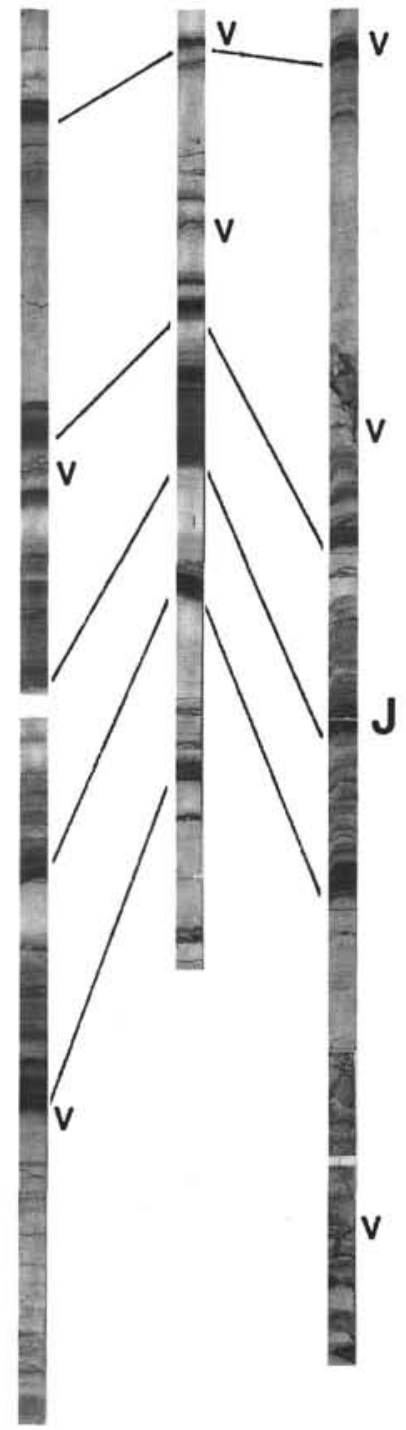

3
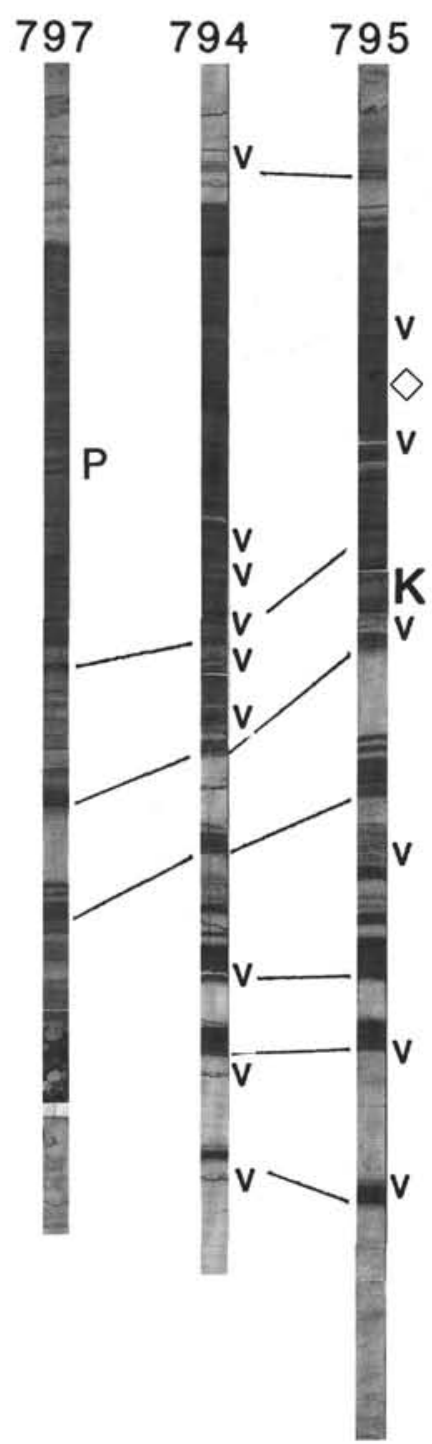

Plate 4. 1. Intervals from 797B-4H-2, $0 \mathrm{~cm}$, to $797 \mathrm{~B}-4 \mathrm{H}-3,150 \mathrm{~cm}, 794 \mathrm{~A}-2 \mathrm{H}-7,7 \mathrm{~cm}$, to $794 \mathrm{~A}-3 \mathrm{H}-1,115 \mathrm{~cm}$, and $795 \mathrm{~A}-3 \mathrm{H}-4,30 \mathrm{~cm}$, to $795 \mathrm{~A}-3 \mathrm{H}-5,150 \mathrm{~cm}$. 2. Intervals from $797 \mathrm{~B}-4 \mathrm{H}-4,0 \mathrm{~cm}$, to $797 \mathrm{~B}-4 \mathrm{H}-5,150 \mathrm{~cm}, 794 \mathrm{~A}-3 \mathrm{H}-1,115 \mathrm{~cm}$, to $794 \mathrm{~A}-3 \mathrm{H}-3,20 \mathrm{~cm}$, and $795 \mathrm{~A}-3 \mathrm{H}-5,150 \mathrm{~cm}$, to $795 \mathrm{~A}-4 \mathrm{H}-1,45 \mathrm{~cm}$. 3 . Intervals from 797B-4H-6, $0 \mathrm{~cm}$, to $797 \mathrm{~B}-5 \mathrm{H}-1,30 \mathrm{~cm}, 794 \mathrm{~A}-3 \mathrm{H}-3,20 \mathrm{~cm}$, to $794 \mathrm{~A}-3 \mathrm{H}-4,130 \mathrm{~cm}$, and $795 \mathrm{~A}-4 \mathrm{H}-1,45 \mathrm{~cm}$, to $795 \mathrm{~A}-4 \mathrm{H}-3,35 \mathrm{~cm}$. 
1

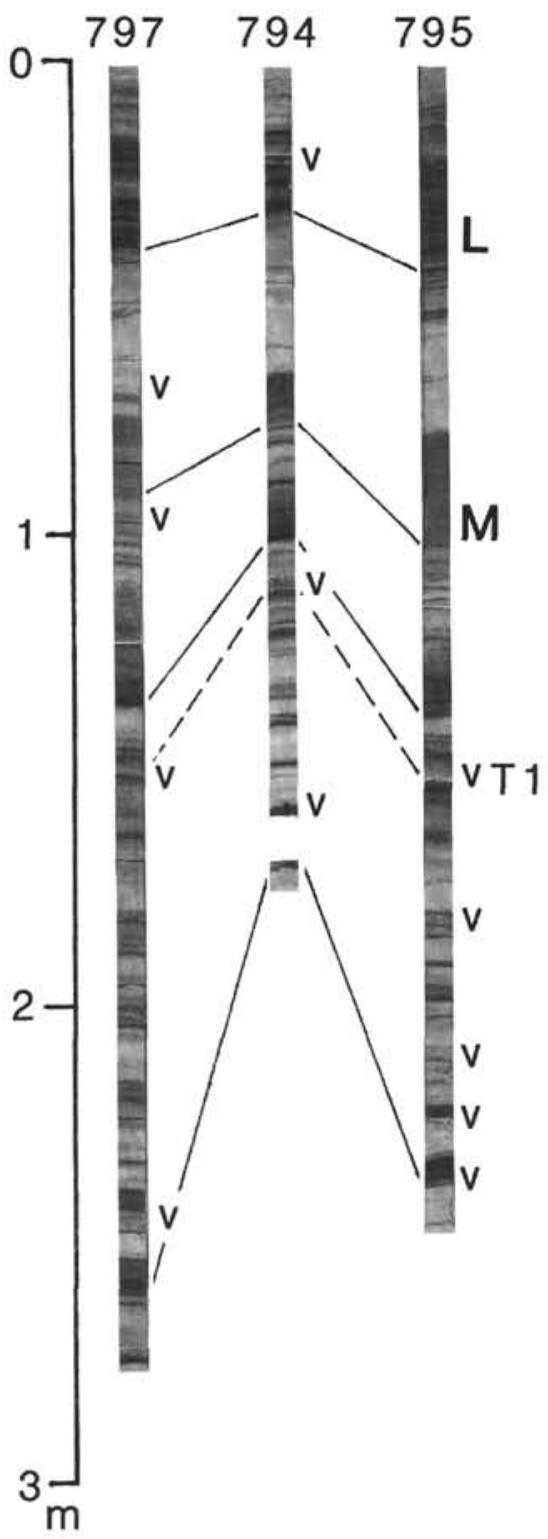

2

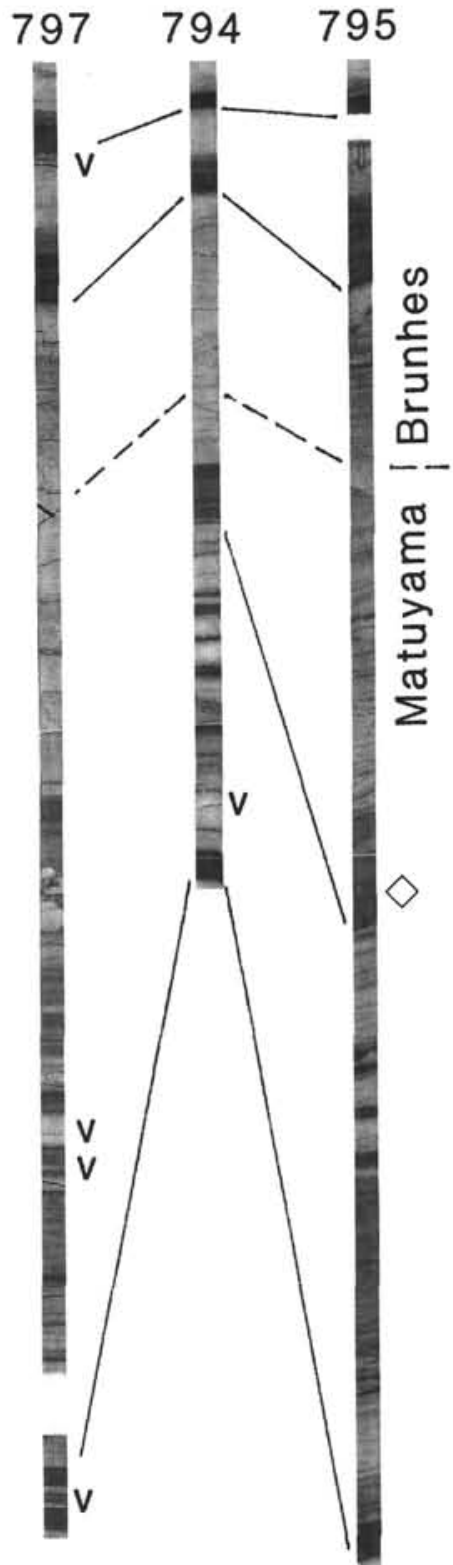

3

$797 \quad 794 \quad 795$

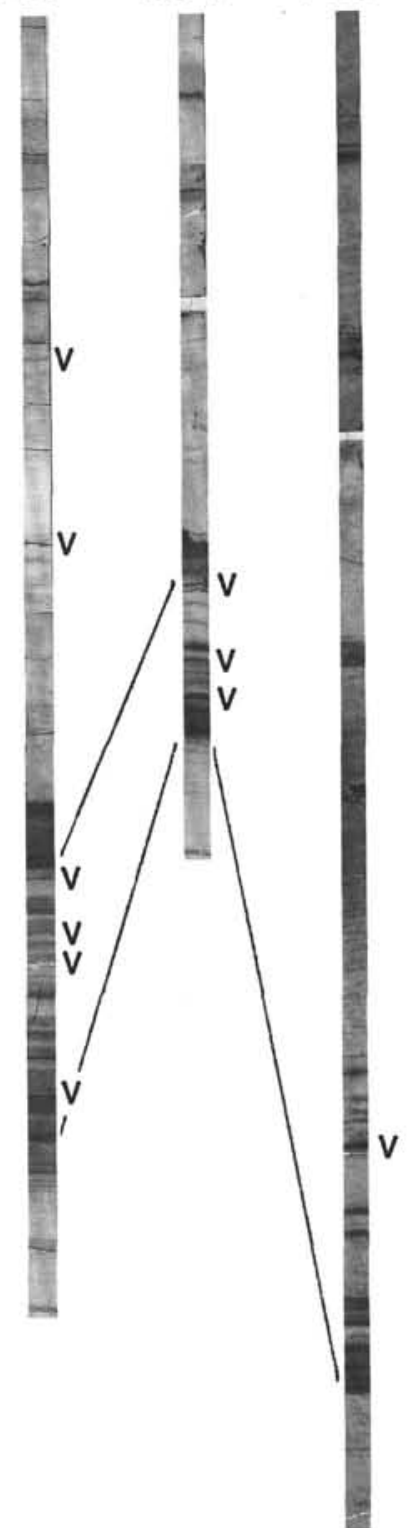

Plate 5. 1. Intervals from $797 \mathrm{~B}-5 \mathrm{H}-1,30 \mathrm{~cm}$, to $797 \mathrm{~B}-5 \mathrm{H}-3,5 \mathrm{~cm}, 794 \mathrm{~A}-3 \mathrm{H}-4,130 \mathrm{~cm}$, to $794 \mathrm{~A}-3 \mathrm{H}-6,5 \mathrm{~cm}$, and $795 \mathrm{~A}-4 \mathrm{H}-3,35 \mathrm{~cm}$, to $795 \mathrm{~A}-4 \mathrm{H}-4,132 \mathrm{~cm}$. 2 . Intervals from 797B-5H-3, $5 \mathrm{~cm}$, to $797 \mathrm{~B}-5 \mathrm{H}-5,24 \mathrm{~cm}, 794 \mathrm{~A}-3 \mathrm{H}-6,5 \mathrm{~cm}$, to $794 \mathrm{~A}-3 \mathrm{H}-7,35 \mathrm{~cm}$, and $795 \mathrm{~A}-4 \mathrm{H}-4,132 \mathrm{~cm}$, to $795 \mathrm{~A}-4 \mathrm{H}-6,150 \mathrm{~cm}$. 3. Intervals from $797 \mathrm{~B}-5 \mathrm{H}-5,24 \mathrm{~cm}$, to $797 \mathrm{~B}-5 \mathrm{H}-6,150 \mathrm{~cm}, 794 \mathrm{~A}-3 \mathrm{H}-7,35 \mathrm{~cm}$, to $794 \mathrm{~A}-4 \mathrm{H}-1,115 \mathrm{~cm}$, and $795 \mathrm{~A}-4 \mathrm{H}-7,0 \mathrm{~cm}$, to $795 \mathrm{~A}-5 \mathrm{H}-2,80 \mathrm{~cm}$. 